\title{
Direct Numerical Simulation of a Tip-Leakage Flow in a Planar Duct with a Longitudinal Slit
}

\author{
Jian Fang¹, Yanfei Gao²*, Yangwei Liu²†, Lipeng Lu² \\ ${ }^{1}$ Scientific Computing Department, Science and Technology Facilities Council (STFC), Daresbury Laboratory, \\ Warrington WA4 4AD, United Kingdom
}

${ }^{2}$ National Key Laboratory of Science and Technology on Aero-Engine Aero-Thermodynamics, School of Energy and Power Engineering, Beihang University, Beijing 100191, China

${ }^{3}$ Faculty of Environment and Technology, Department of Engineering Design and Mathematics, the University of the West of England, Bristol, BS16 1QY, United Kingdom

${ }^{4}$ Laboratoire de Mécanique Des Fluides (LMFA), Ecole Centrale de Lyon (ECL), CNRS Unité Mixte de Recherche (UMR) 5509, 36, avenue Guy de Collongue, 69130 Ecully, France

ABSTRACT: A planar duct flow configuration with a cross-flow injected from a longitudinal slit close to the upper wall of the duct is studied by using a direct numerical simulation approach to explore the underlying flow mechanism in relation to the tip-leakage vortex (TLV), which is one of the most important flow phenomena in turbomachinery. Major characteristics of TLV in a rotor of turbomachinery are identified in the current flow model. The analysis of mean and instantaneous flow fields reveals that the interaction between the main (axial) flow and jet (cross) flow is the primary source of the generation of the TLV. The evolution of the TLV is then investigated, and a vortex breakup phenomenon is identified. The evolution of TLV can be divided into three phases, i.e. the formation phase, the break-up phase, and the diffusion phase. Mean streamlines and turbulence kinetic energy (TKE) budgets are analysed, showing that the high TKE central spot in the formation phase is due to the interaction between highly swirling vortex filaments and mean velocity gradient. In the outer part of the TLV, the TKE is mainly produced in the shear-layer and transported towards the centre by the turbulence transport.

Keywords: Tip-leakage vortex; Direct-numerical simulation; Wall-jet; Turbomachinery flow.

\footnotetext{
* Corresponding author: yanfeigao@buaa.edu.cn

† Corresponding author: liuyangwei@126.com

$\$$ Prof. Lu unfortunately passed away before the submission of the paper.
} 


\section{Introduction}

Turbomachinery is a core component of the modern energy transfer system, such as a heavy-duty gas turbine for power generation and a jet-engine for aviation. It is critical to study turbomachinery aerodynamics with a view to improving its performance. Tip-leakage flow (TLF) is a typical secondary flow phenomenon in turbomachinery, which is moving across the tip clearance between the blade tip and the casing, driven by the pressure difference between the pressure surface and the suction surface of the blade. It is considered as one of the three major sources of the loss in turbomachinery [1], along with the profile loss and secondary loss. It also has overall effects on turbomachinery's performance, such as efficiency ${ }^{[2,3]}$, noise generation ${ }^{[4]}$ and stability ${ }^{[5,6,7]}$. Downstream of the tip clearance, the TLF rolls up to form unsteady vortical structures, brings vorticity into the flow passage, and further interacts with the main (axial) flow inside the blade passage. Hence, it is of great significance for the energy and aerospace industries to investigate the dynamics and mechanisms of TLF.

Because of the complexity and non-equilibrium nature of TLF, the Reynolds-averaged Navier-Stokes (RANS) simulation in the tip region is generally unsatisfactory ${ }^{[1,8]}$, although RANS has been widely adopted in the turbomachinery industry as a productive computational fluid dynamics (CFD) tool [9]. Turbulence models used in RANS approach are the main source of the inaccuracy and uncertainty of CFD [10], as most turbulence models are semi-empirical, and those constants and coefficients in eddy viscosity-based models are mostly calibrated with experimental data of basic equilibrium turbulent flows [11]. Therefore, RANS often fail to predicate a correction mean flow field in the tip region, where the turbulence is in a typical non-equilibrium state [12]. Further improvement of turbulence models in TLF requires a deeper understanding of turbulence mechanism of tip-leakage vortex (TLV) and accurate flow field data for calibration of parameters.

Numerous experiments have been done in the past to investigate TLF, producing a large amount of dataset of pressure distribution, velocity, vorticity, and Reynolds stress at specific measurement points. Recently, advanced experimental technologies like stereo particle image velocimetry (SPIV) have been used to acquire detailed velocity field of TLV. For example, Yu and Liu ${ }^{[13]}$ and Du et. al. ${ }^{[14]}$ investigated the TLV in an axial compressor rotor passage using SPIV, and revealed many important features of the TLV, including the breakdown of the TLV, the formation of the compound corner vortex, and the evolution of the tip corner antirotating streamwise vortices. The comprehensive SPIV data recently obtained by Miorini et al. [15] and Wu et al. 
[16] in an axial water pump showed very complex turbulence structures inside a TLV. They reported the mismatch between turbulence kinetic energy (TKE) and its production and pointed out that the TKE peak at the TLV's core is due to the convection effect. Researchers were trying to understand the underlying mechanism of the TKE production and its transport characteristics of TLV [15-17]. However, it is still quite difficult to reconstruct a detailed three-dimensional (3D) flow field using experimental data, which limits a further investigation of TLV.

Compared to RANS, eddy-resolved numerical methods, such as direct numerical simulation (DNS), largeeddy simulation (LES) and hybrid RANS/LES, have been demonstrated to yield much more accurate, reliable and detailed data [18], which greatly benefits the research in flow physics and validation of turbulence models [19,20]. You et al. [21,22,23] have conducted a LES of a linear cascade flow with a tip clearance to investigate the clearance size effect, viscous loss and vortex dynamics of the TLF. Their research suggests the endwall viscous

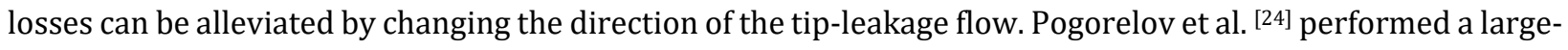
scale LES on the TLF of an axial fan rotor. The detailed flow fields in the rotor tip region were resolved, and the turbulence and acoustics characteristics were investigated. Moghadam et al. ${ }^{[25]}$ studied the TLV in a model axial fan at different tip-gap sizes and operating conditions, using a LES approach. Good agreements with the experimental measurements were achieved, and the breakdown of the TLV was captured in a large tip-gas size case. Zonal-LES [26,27] and delayed detached-eddy simulation (DDES) ${ }^{[28,29]}$ were then employed in the TLF problem, showing that hybrid RANS/LES methods are also feasible approaches for the problem with an acceptable computational cost. It must admit that DNS is still the most favourite tool for fundamental turbulence research because all turbulent scales are resolved without introducing any empirical parameter [30], although it is too computationally expensive to apply directly to a full-scale engineering problem at a working condition. Even the LES approach was only applied for model cascades $[21,22,23]$ and rotors $[25]$, rather than fullsize turbomachinery. Therefore, it is more realistic to apply DNS in a more generalised flow model extracted from an engineering problem. Such a model shall have a simple geometry and work at a relatively low Reynolds number, but contain major flow features and key characteristics of turbulence for a study of physics of fluids. A jet model was proposed by Rains ${ }^{[31]}$ to study the mechanism of loss due to blade tip clearances. A compound vortex model was used by Lakshminarayana [32], and the blade-to-blade variation and stagnation pressure losses in TLF were well predicted. Chen et al. ${ }^{[33]}$ presented a similarity analysis by decomposing TLF into 
independent throughflow and crossflow, and their calculations based on the similarity scaling agreed well with the experimental data. Recently, Wheeler et al. ${ }^{[34,35,36]}$ developed a channel configuration with a sharp inlet corner to model flow over the tip of a turbine blade. DNS is adopted to resolve the details of the development of the turbulence boundary layer in the tip area, together with the study of the tip flow aerodynamics and heat transfer under the influences of free-stream turbulence, cross-flow and the pressure side boundary layer.

Inspired by Chen et al. [33], a generalised TLF model is adopted to represent the cross-jet flow/passage flow interaction and the TLV roll-up. The TLF is reduced to a wall-jet flow ejected from a slit on a sidewall of a cuboid duct, and the wall-jet interacts with the passage flow, creating a helical rolling-up vortex for the research of TLV. The present flow model can be regarded as a combination of two basic types of jet flows, i.e. the wall jet [37] and jet in crossflow [38]. It is a simple flow model compared with a rotor in turbomachinery yet still with generality. Meanwhile, it still contains complex three-dimensional vortical structures observed in rotors. The flow physics based on the analysis of the flow model can be used to understand the vortex dynamics in turbomachinery, and the DNS database produced can be used to improve turbulence models, especially for those emerging data-driven turbulence modelling methods [39,40]. The previous LES studies ${ }^{[41,42]}$ have shown that the flow model could reproduce similar flow field with the TLV in a compressor rotor. Apart from its simplicity, flexibility is also favourable. Some key factors in the TLF, such as gap geometry, adverse pressure gradient and relevant motion of casing wall, can be added to the basic form of the flow model and studied individually or jointly. A DNS approach is employed in the present study to simulate the model flow at a low Reynolds number. The statistics in the duct flow without the cross-jet flow is validated first. Then the DNS data are analysed, focusing on flow structures and turbulence mechanisms of the TLV, especially the TKE production mechanisms in the vortical region.

\section{Governing Equations and Computational Setup}

\subsection{Governing Equations}

The 3D unsteady compressible Navier-Stokes (N-S) equations given as,

$$
\begin{gathered}
\frac{\partial \rho}{\partial t}+\frac{\partial \rho u_{i}}{\partial x_{i}}=0 \\
\frac{\partial \rho u_{i}}{\partial t}+\frac{\partial \rho u_{i} u_{j}}{\partial x_{j}}-\frac{\partial p}{\partial x_{i}}=\frac{\partial \sigma_{i j}}{\partial x_{j}}
\end{gathered}
$$




$$
\frac{\partial \rho E}{\partial t}+\frac{\partial \rho E u_{j}+p}{\partial x_{j}}=-\frac{\partial q_{j}}{\partial x_{j}}+\frac{\partial u_{i} \sigma_{i j}}{\partial x_{j}}
$$

are numerically solved, in which the primary variables are the density $\rho$, the pressure $p$, the velocity component $u_{i}$ with $\left(u_{1}=u, u_{2}=v, u_{3}=w\right)$, and the total energy per unit mass $E$, defined as, $E=\frac{p}{(\gamma-1) \rho}+\frac{1}{2} u_{i} u_{i}$, with the ratio of specific heats $\gamma=1.4$.

Based on a Newtonian fluid, the viscous stress tensor $\sigma_{i j}$ is defined as,

$$
\sigma_{i j}=\mu\left(\frac{\partial u_{i}}{\partial x_{j}}+\frac{\partial u_{j}}{\partial x_{i}}\right)-\frac{2}{3} \mu \frac{\partial u_{k}}{\partial x_{k}} \delta_{i j}
$$

where $\mu$ is the dynamic viscosity and calculated via the Sutherland law as,

$$
\mu=T^{1.5} \frac{T_{S} / T_{0}+1}{T+T_{S} / T_{0}}
$$

where $T$ is the temperature, $T_{0}=298.15 \mathrm{~K}$ and $T_{S}=110.4 \mathrm{~K}$.

The heat flux vector is defined according to the Fourier's law as,

$$
q_{i}=-\lambda \frac{\partial T}{\partial x_{i}}
$$

where $\lambda=\mu c_{p} / \operatorname{Pr}$ is the coefficient of thermal conductivity, $\operatorname{Pr}=0.72$ is the Prandtl number, $C_{P}=\gamma R /(\gamma-1)$ is the specific heat capacity of the gas at constant pressure with the gas constant, $R=287.1 \mathrm{~J} /(\mathrm{K} \cdot \mathrm{kg})$.

\subsection{Numerical Method}

By Jacobian transformation and conformal mapping, Eqs. (1-3) are projected to a computational domain to deal with the local mesh deformation, and the transformed N-S equations are solved within a CFD framework of high-order finite difference method by using an in-house code ASTR, which has been previously applied to DNS ${ }^{[43,44,45]}$ and LES ${ }^{[46,47,48]}$. The $6^{\text {th }}$-order compact central scheme ${ }^{[49]}$ is adopted to solve both the convection and diffusion terms. The compact scheme is decoupled at the interface between two neighbouring subdomains to adapt a domain-decomposition based parallel computation [50]. The $2^{\text {nd }}$ derivatives of the diffusion terms are solved with the application of the first-order difference twice in a successive manner. This method is more efficient than the direct calculation of the second-order derivatives although the latter method could be numerically more stable ${ }^{[51]}$. To remove the small-scale numerical wiggles due to aliasing errors resulting from the discrete evaluation of the nonlinear convection terms, a $10^{\text {th }}$-order compact low bypass filter is 
incorporated, which limits the filtering only at high wavenumbers ${ }^{[52]}$. After all the spatial terms are solved, a third-order total variation diminishing (TVD) Runge-Kutta method [53] is used for the time integration.

\subsection{Domain and Mesh}

The computational domain is an $8 l_{0} \times 2 l_{0} \times 1 l_{0}$ cuboid, where a reference length $l_{0}$ is the same as the spanwise width. An extra sponger layer is added at the end of the domain to minimise the reflection of numerical errors from the outlet boundary. The slit is defined as a zone of $x \geq 2,1.95 \leq y \leq 2$ and $z=0$, shown as the blue patch in Figure 1. A mesh of $512 \times 384 \times 192$ nodes is used to discretise the domain. As represented in Figure 1, the mesh is uniformly distributed in the $x$ direction within the effective domain and hyperbolically stretched in the sponge layer. In the $z$ and $y$ directions, the mesh is concentrated towards all four walls to enhance the resolution of the near-wall turbulence. No further special treatment is needed for the slit area since the mesh is already fine in that region. The mesh resolutions in wall unit based on the inflow properties are listed in Table 1, from which it can be seen that the streamwise mesh resolution in wall unit $\Delta x^{+}$is below 10 and the radial and transverse mesh resolutions $\Delta y^{+}$and $\Delta z^{+}$varies from 0.75 at the wall to 5.82 in the centre of the duct. These resolutions satisfy the criteria of DNS of a wall turbulence suggested by Sagaut [54]. The ratio of the effective mesh size (defined as $\Delta=\sqrt[3]{\Delta x \Delta y \Delta z}$ ) to the local Kolmogorov scale $\eta$ calculated based on the inflow turbulence is plotted in Figure 2. The value of $\Delta / \eta$ is below 4 on the inflow plane, ensuring that the mesh resolution is down to the smallest turbulence scale in the present DNS study.

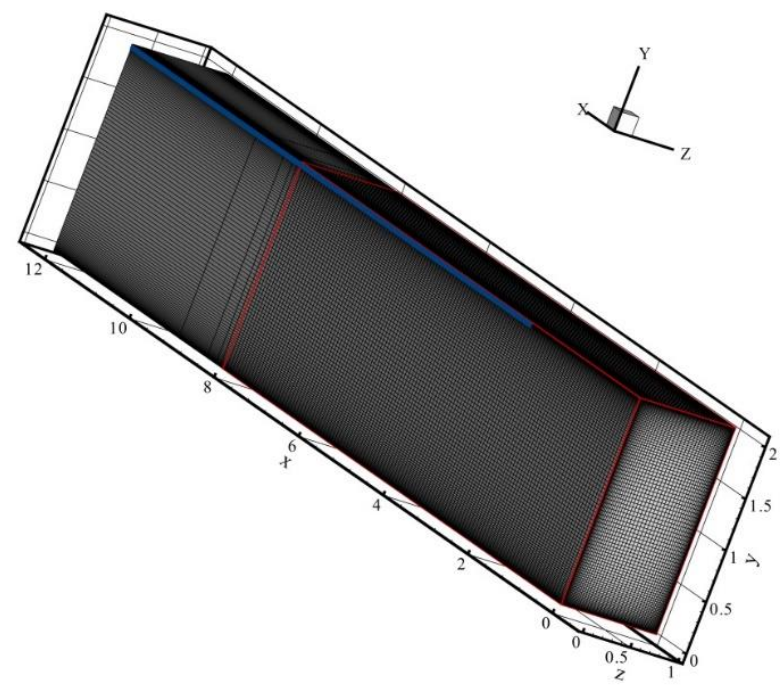

Figure 1. A sketch of the computational domain along with computational meshes. The mesh nodes are drawn every 4 points and the red solid lines mark the effective domain. The blue patch denotes the slit. 
Table 1. Mesh resolution at the inflow plane*

\begin{tabular}{ccccc}
\hline$\Delta x^{+}$ & $\Delta y_{1}^{+}$ & $\Delta y_{m}^{+}$ & $\Delta z_{1}^{+}$ & $\Delta z_{m}^{+}$ \\
\hline 9.64 & 0.74 & 5.82 & 0.75 & 5.82 \\
\hline
\end{tabular}

${ }^{*} \Delta y_{1}^{+}$and $\Delta z_{1}^{+}$represent the mesh spaces of the first node away from the wall in the local wall unit. $\Delta y_{m}^{+}$and $\Delta z_{m}^{+}$are the mesh resolutions at the centre of the duct. The wall variables are based on the inflow turbulence properties.

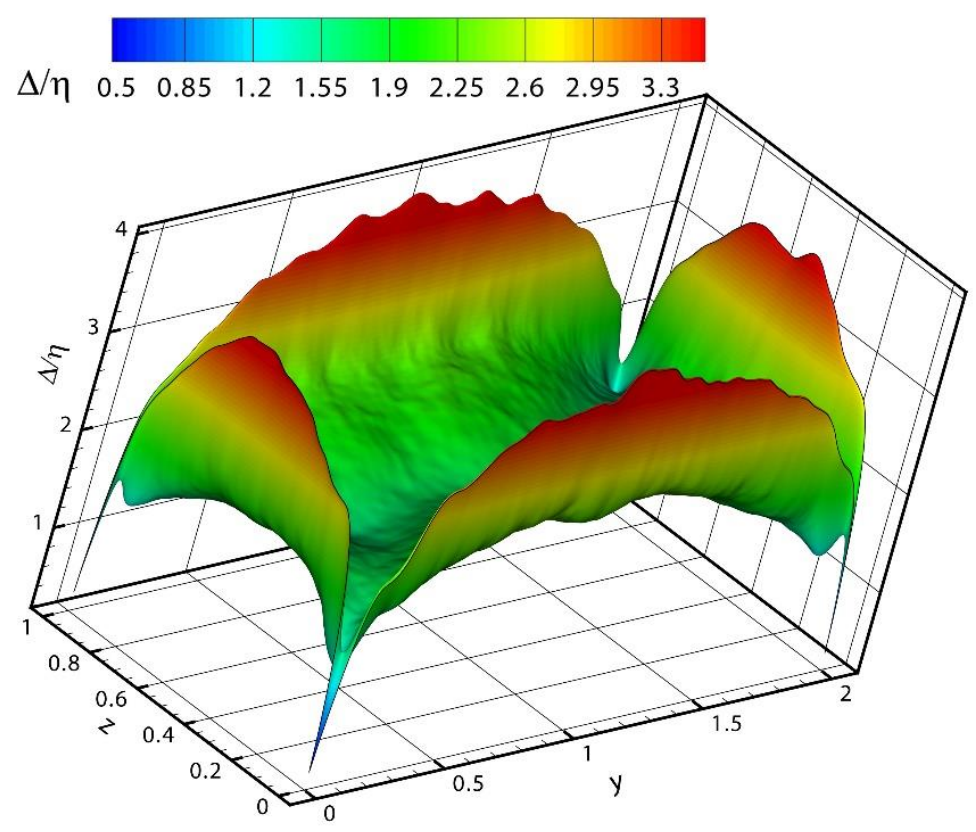

Figure 2. The ratio of the effective mesh size $(\Delta)$ to the local Kolmogorov scale $\eta . \eta$ is calculated based on the inflow turbulence properties.

\subsection{Computational Setup}

The flow is primarily a subsonic turbulent duct flow with an inflow Mach number $M a=u_{0} / \sqrt{\gamma R T_{0}}=0.5$ and Reynolds number $R e=\rho_{0} u_{0} l_{0} / \mu_{0}=10,000$, in which $u_{0}$ is the reference velocity taken as the bulk averaged inflow velocity, $\rho_{0}$ is the reference density taken as the bulk averaged density of the inflow, $T_{0}$ is the reference temperature taken as the wall temperature, and $\mu_{0}$ is the reference viscosity calculated based on $T_{0}$. The corresponding Reynolds number based on friction velocity is $R e_{\tau}=600$, which is much lower than the Reynolds number of turbomachinery at a working condition. Therefore, the study of the present paper is meant for a qualitative analysis of TLV dynamics in turbomachinery.

To generate appropriate inflow turbulence, a precursor DNS of a turbulent duct flow with the homogeneous $x$-direction applying the periodic condition at $x=0$ and $x=x_{\max }$ planes is conducted. An unsteady uniform body force proposed by Lenormand et al. ${ }^{[5]}$ is adopted to drive the flow inside the duct to maintain a constant 
mass flux. After the turbulence in the duct being fully developed, instantaneous flow field at a $x$-location is extracted and saved at every 20 time steps as the inflow database for the main simulation. The generated inflow data slices are introduced to the main flow simulation at the $x=0$ plane via the subsonic inflow boundary condition of Rudy and Strikwerda [56]. At the outflow plane $\left(x=x_{\max }\right)$, the non-reflective outflow condition [57] is used. A non-slip isothermal condition with wall temperature equalling to $T_{0}$ are applied at $y=0, y=$ $y_{\max }, z=0$ and $z=z_{\max }$ planes, except for the slit area, where the jet cross-flow is introduced into the duct with the subsonic inflow condition with a uniform spanwise inlet flow $w_{i n}=1.5 u_{0}$.

The time step for the main simulation is set to be $\Delta t=0.45 \times 10^{-3} t_{0}$, where $t_{0}=l_{0} / u_{0}$ is the reference time scale, corresponding to a CFL number of 0.99 . The main simulation has been persisted till $t=113.5 t_{0}$, which is about 14 through-flow passing the computational domain and the statistics are collected every 40 time steps from $t=37.18 t_{0}$ after the simulation has reached a statistically steady status, and 4240 samples are collected to calculate statistics.

\section{Results and Discussion}

\subsection{Validation}

The flow statistics in the inflow plane is first analysed to validate the simulation. The mean streamwise velocity, $\langle u\rangle$, and the Reynolds stress components, $\left\langle u_{i}^{\prime \prime} u_{j}^{\prime \prime}\right\rangle$, are shown in Figure 3, and \langle\rangle and the superscript, ", stand for Favre-averaged mean variables and fluctuations, respectively. From Figure 3, a near symmetrical flow field along both $y$ and $z$ directions can be observed. Mean velocity profile and Reynolds stresses in the local wall unit along the central lines (marked as a dashed line in Figure 3 a) are presented in Figure 4, and the DNS of an incompressible planar channel flow at $\operatorname{Re}_{\tau}=590$ of Moser et al. [58] is used for a reference. Good agreement can be observed in the linear and logarithmic layers, where the local wall turbulence dominates the flow. Close to the centre of the duct, the velocity profile presents a wake layer, which was also reported in a recent DNS of turbulent flow in a square duct [59]. The mesh sensitivity of the flow in the duct without a slit is also checked (data not shown), and the mean flow field and Reynolds stress are confirmed to be independent of the mesh's resolution. 


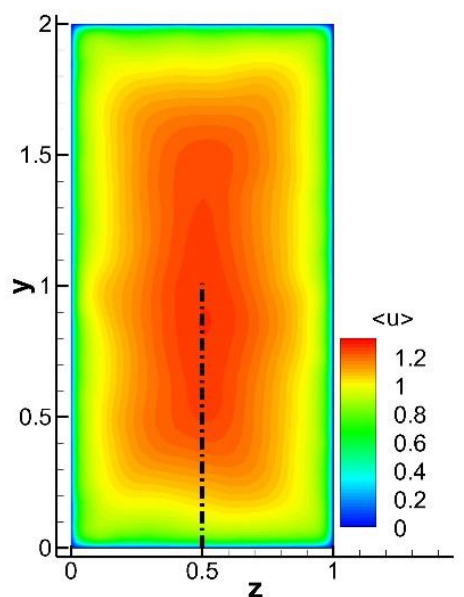

(a)

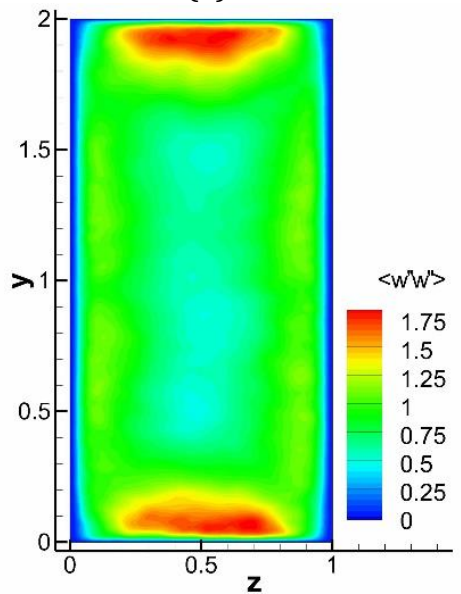

(d)

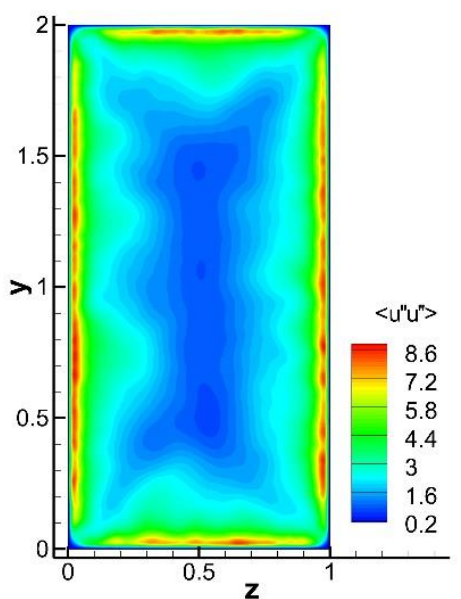

(b)

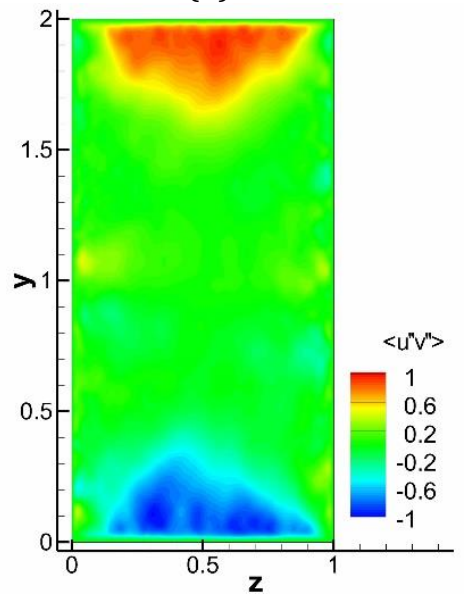

(e)

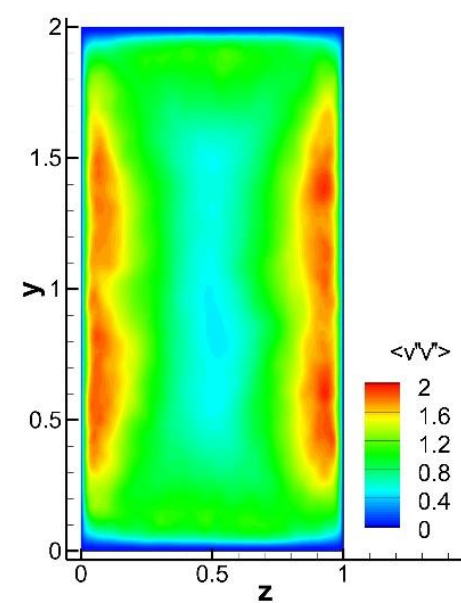

(c)

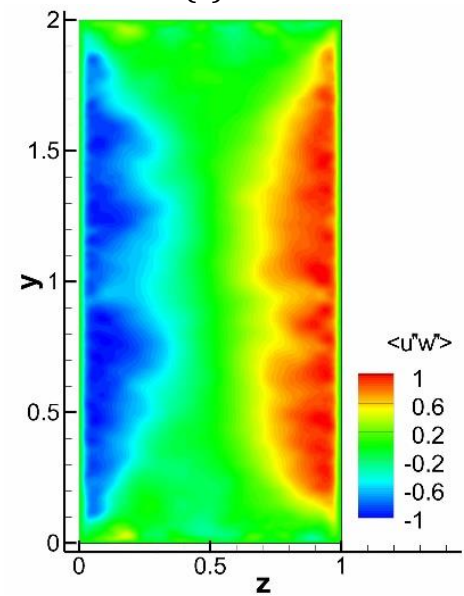

(f)

Figure 3. Mean streamwise velocity and Reynolds stress components of the inflow turbulence. (a): $\langle u\rangle$; (b): $\left\langle u^{\prime \prime} u^{\prime \prime}\right\rangle ;(c):\left\langle v^{\prime \prime} v^{\prime \prime}\right\rangle ;(d):\left\langle w^{\prime \prime} w^{\prime \prime}\right\rangle ;(e):\left\langle u^{\prime \prime} v^{\prime \prime}\right\rangle ;(f):\left\langle w^{\prime \prime} w^{\prime \prime}\right\rangle .\langle u\rangle$ is normalised with the reference velocity and the Reynolds stresses are normalised with wall units at $(y=0, z=0.5)$.

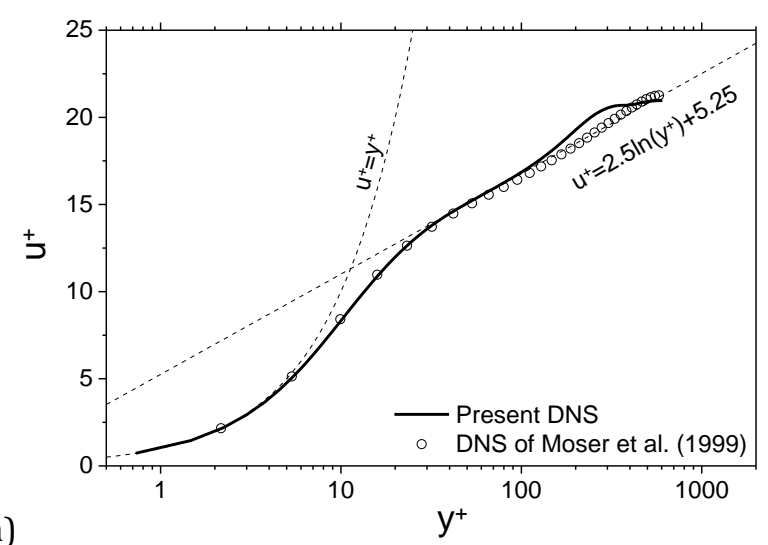

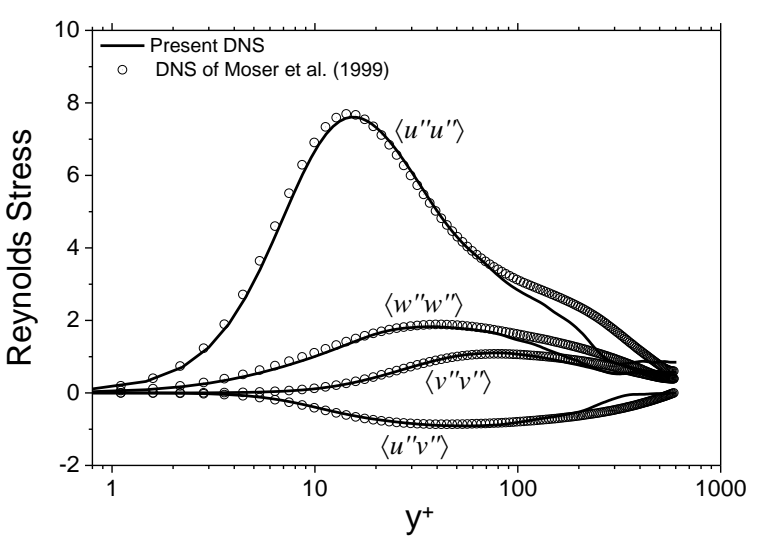

(b)

Figure 4. Profiles of mean velocity (a) and Reynolds stress (b) along the central lines of the inflow plane.

\subsection{Instantaneous flow analysis}


According to the instantaneous velocity field shown in Figure $5(a, b, c)$, we can see that the injection of the spanwise cross flow via the slit, and interaction between the cross jet-flow and the main passage flow forms a spiral-like swirling flow structures with high values of vorticity magnitude shown in Figure 5 (d), namely the TLV. Clearly, the swirling motion of TLV induces strong momentum exchanges across the upper part of the duct. Consequently, a large area of low-speed flow region is produced, and the velocity close to the centre of the duct is increased due to the blockage effect, as reflected in Figure 5 (a). The initial formulation of TLV as well as its evolution and break-up can be observed from Figure 5 (d) and is also shown in details in Figure 6, from which we can see the concentrated positive vorticity at a position of $x=2.5$ breaks up into small-scale vortices at a position of $x=7$, occupying up to $1 / 3$ of the cross-section.

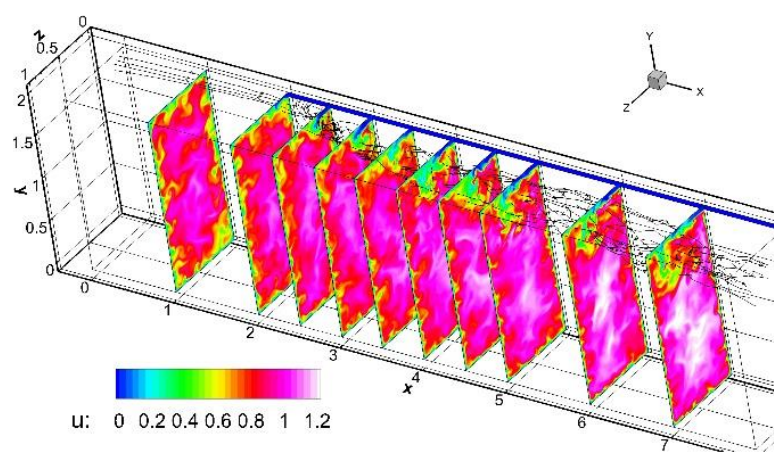

(a)

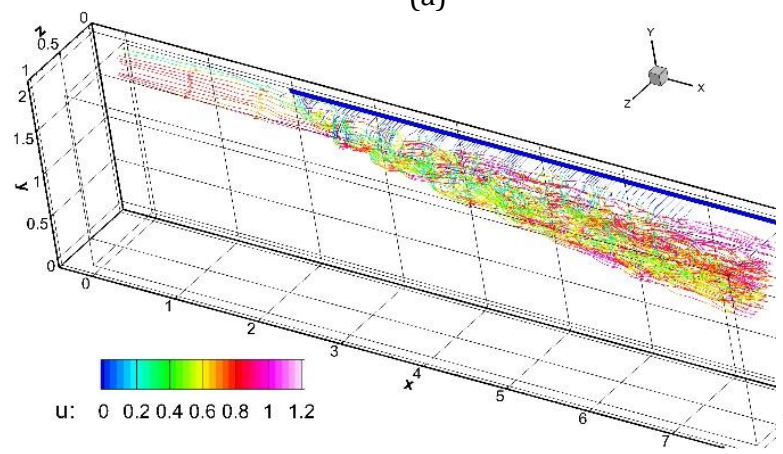

(c)

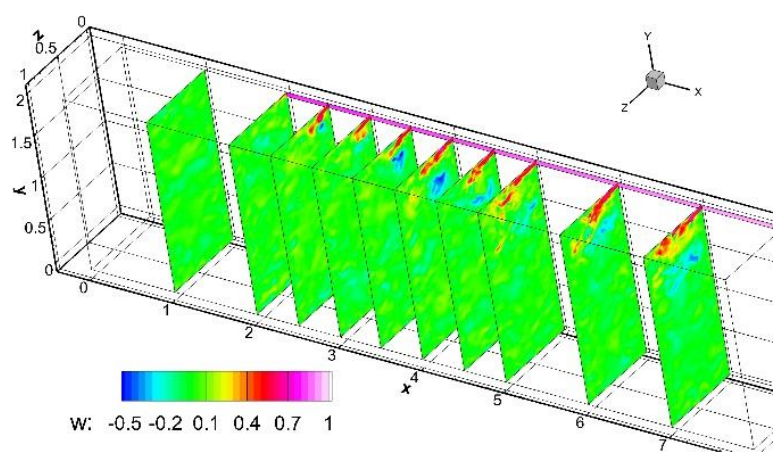

(b)

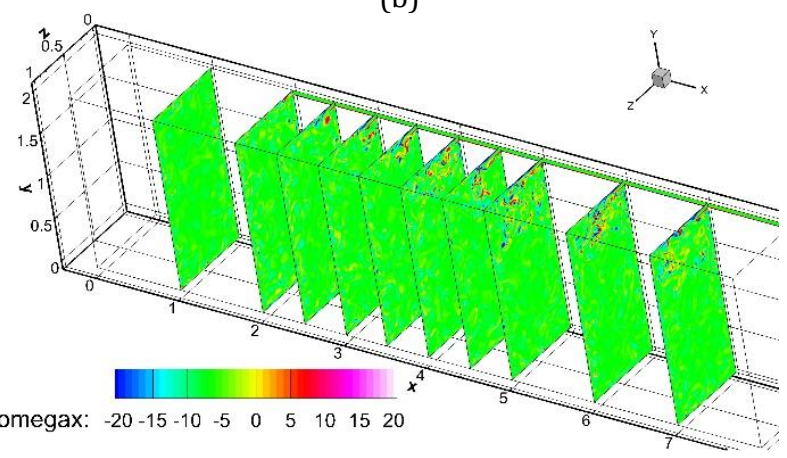

(d)

Figure 5. Instantaneous streamwise velocity $u$ (a), spanwise velocity $w$ (b), streamlines (c) and stream vorticity $\omega_{x}$ (d) on 10 selected $z-y$ planes at $t=113.5$. The locations of the selected $z-y$ planes are at $x=1,2,2.5,3,3.5,4,4.5,5,6,7$. 

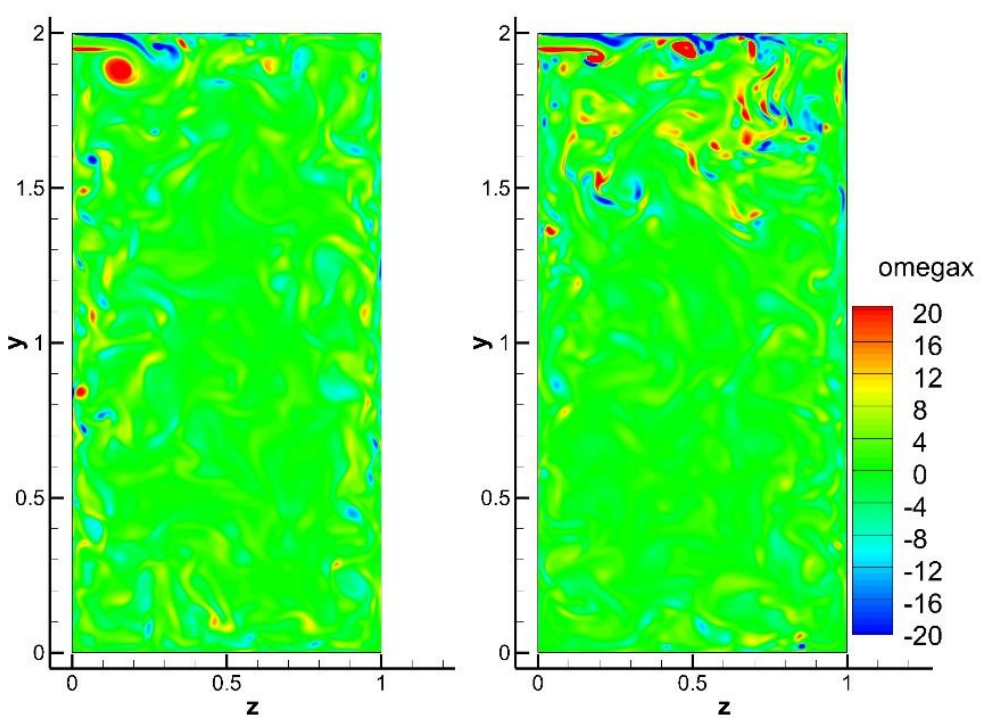

Figure 6. Streamwise vorticity $\omega_{x}$ on the $x=2.5$ (left) and $x=7$ (right) slices.

The instantaneous turbulence structures visualised with the $\lambda_{c i}$ criteria (i.e. the swirling strength ${ }^{[60,61]}$ ) are presented in Figure 7. In the initial stage of the formation of the vortex, a relatively concentrated vortex structure can be identified, and it breaks up between $x=2.6$ and $x=2.8$ as highlighted in Figure 7. After the initial break-up, TLV can be regarded as a package of many small-scale vortical structures. With the development of TLV downstream, the vortical package approaches the wall opposite to the slit $\left(\mathrm{z}=\mathrm{Z}_{\max }\right)$ and interacts with the spatially-developing wall turbulence there. 


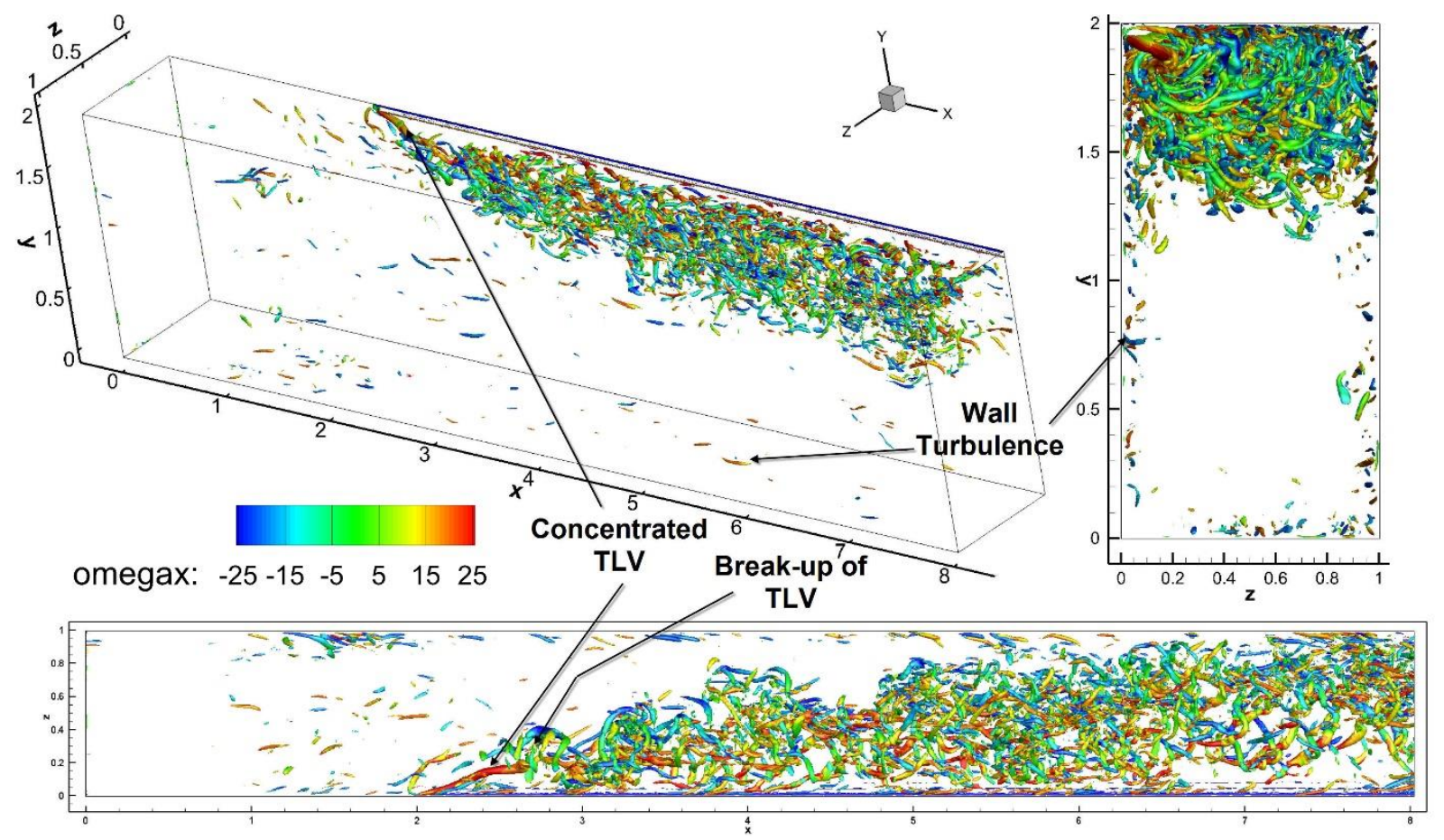

Figure 7. Instantaneous turbulence coherent structures with different angles of view. The coherent structures are visualised by the iso-surface of $\lambda_{c i}$ equalling to $0.2 \%$ of its global maximum and coloured with streamwise vorticity.

The iso-surfaces of the instantaneous vorticity magnitude $|\omega|=\sqrt{\omega_{x}^{2}+\omega_{y}^{2}+\omega_{z}^{2}}$, which indicates the shear flow strength in different regions, are presented in Figure 8. Upstream of the slit, the shear-layer is basically a streamwise elongated streaky flow structure which is the typical characteristic of wall turbulence. The jet from the slit induces a strong shear-layer close to the top wall $\left(y=y_{\max }\right)$ with negative values of $\omega_{x}$ as shown in Figure 8 (a). Downstream, the shear-layer penetrates towards the $z_{\max }$ wall boundary layer, and the front of the shear-layer becomes more unstable. In the meantime, the jet pushes the 'streaky' flow structures towards the $z_{\max }$ wall, and makes them more twisted. The interactions between the jet flow from the slit and the wall turbulence from the top wall $\left(y=y_{\max }\right)$ also generates small-scale flow structures around their interfaces as shown in Figure 8 (a) and (b). A positive $\omega_{x}$ shear-layer is formed from the lower lip of the slit due to the shear between the spanwise jet and the main passage flow, as shown in Figure 8 (b) and (c). The positive $\omega_{x}$ shearlayer is clearly more unstable than the negative $\omega_{x}$ shear-layer above it, and its penetrating depth is also much shorter. Close to the centre of the duct, the shear-layer is present as a large number of small-scale flow structures (shown in Figure 8 (d)) which is co-existing in the main body of the TLV. 
(a)

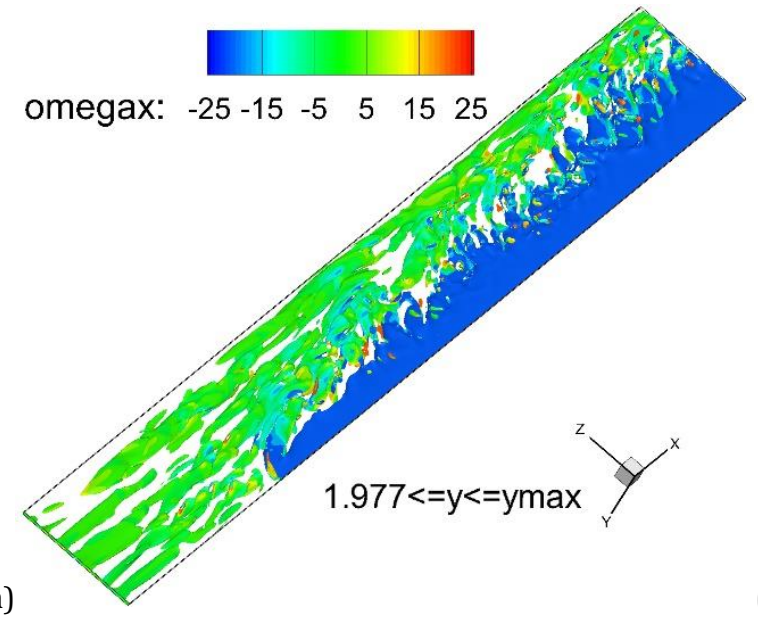

(b)

(c)

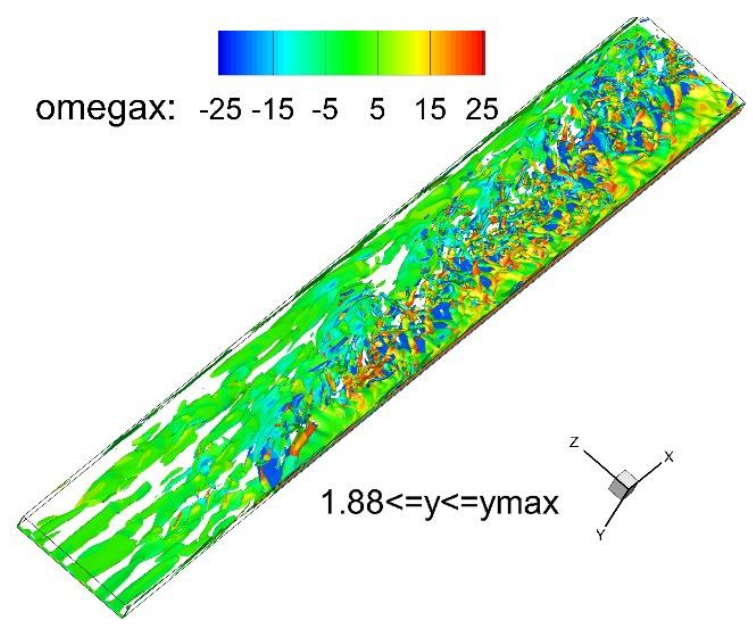

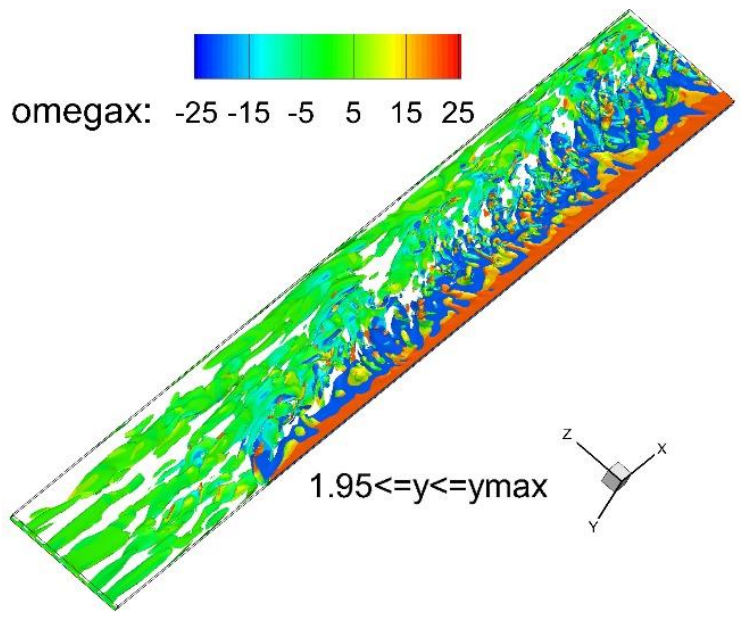

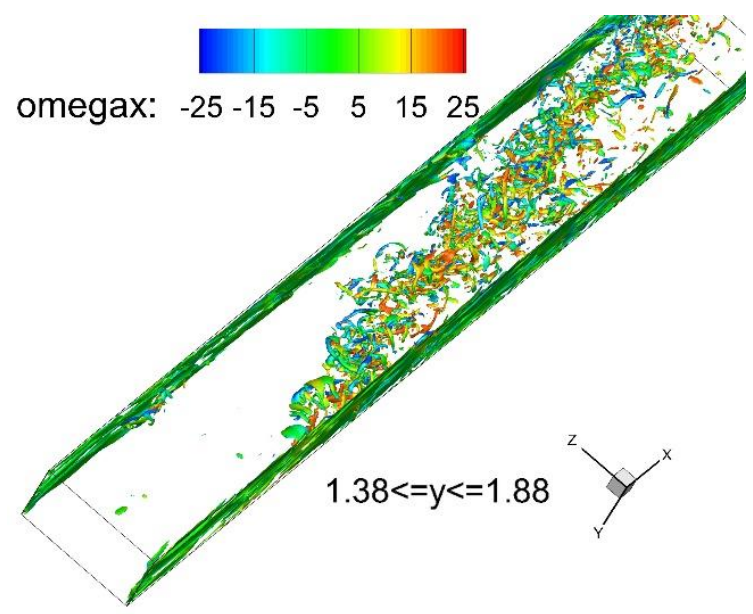

Figure 8. Instantaneous turbulence coherent structures visualised by the iso-surface of $|\omega|$ equalling to $35 \%$ of its global maximum and coloured with streamwise vorticity. (a, b, c) show structures close to the $\boldsymbol{y}_{\max }$ wall, and (d) shows structures away from the wall.

The instantaneous streamwise velocity fluctuations, $u^{\prime \prime}$, on the two selected $z-x$ slices are shown in Figure 9, from which we can see the interactions of the jet and the wall turbulence from the $y=y_{\max }$ plane. Upstream of the slit, the near-wall area is filled with typical streamwise elongated streaks, which was so-called well organised structures of wall turbulence first reported by Kine et al. [62]. In contrast, the jet flow from the slit is quiet and barley fluctuating, due to the uniform inflow boundary condition applied on the slit. The jet flow then pushes the streaky structures towards the $z=Z_{\max }$ wall, and the shape of streaks is highly twisted. Meanwhile, the jet front becomes unstable and breaks up into smaller flow structures. Also, we can observe a transitional area between less fluctuating jet flow and streaky wall turbulence, and the flow in the transitional area is more isotropic and chaotic. 
(a)

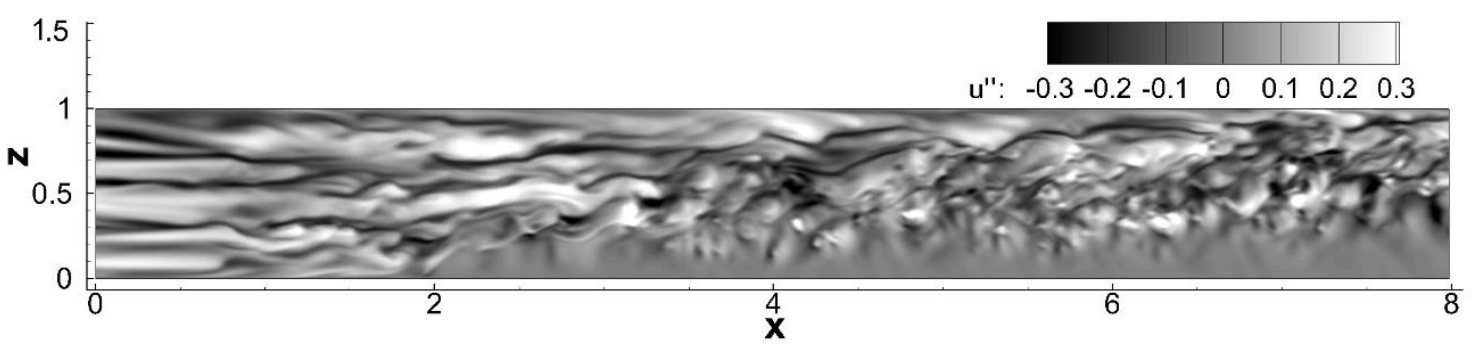

(b)

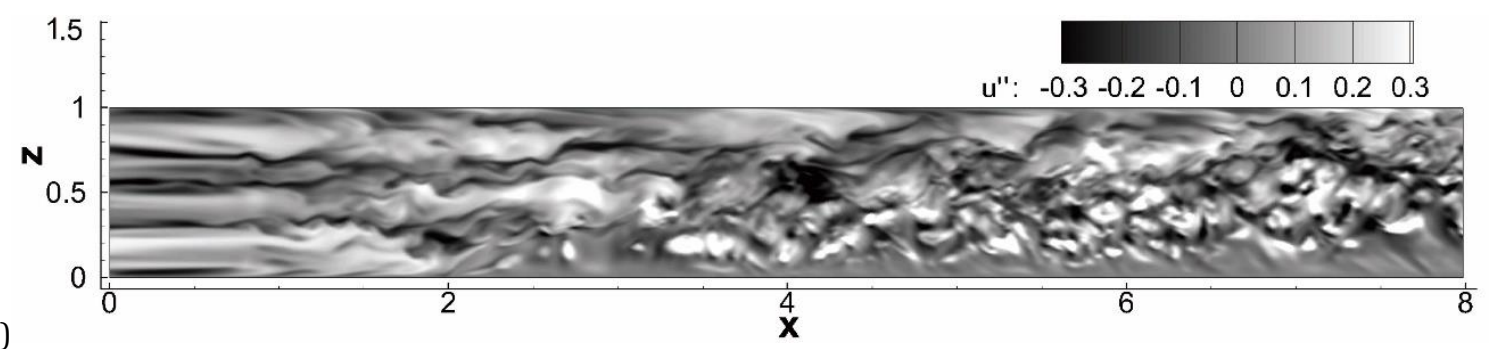

Figure 9. Instantaneous streamwise velocity fluctuations at two x-z planes close to the top wall. (a) locates at $y=1.975$; (b) locates at $y=1.95$;

\subsection{Mean flow statistics}

The time-averaged streamwise velocity field with mean streamlines is shown in Figure 10, from which we can see the development of the low-speed zone due to the mixing effect of the TLV with the passage flow. Close to the centre of the duct, the flow speed gets increased as the consequence of the blockage effect of the TLV and the increase of the mass flux from the injected jet. The jet from the slit bends towards the streamwise direction, swirls around the TLV core and mixes with the main passage flow. The mean $y$-direction velocity $\langle v\rangle$ shown in Figure 10 (b) shows positive values in the left-hand side of the duct and negative values in the right-hand side of the duct (assuming the normal direction is $+x$ ), presenting the mean vortical motion of the TLV. The mean spanwise velocity $\langle w\rangle$ in Figure 10 (c) presents the strong positive spanwise jet velocity from the slit near the top wall and the relatively negative spanwise velocity region away from the top wall, also indicating a vortical motion of the mean flow. Considering the TLV is in mainly alignment with the $x$-direction and the flow is weakly compressible, the centre of the TLV is defined as the local minimum of the 2D stream function, $\varphi(z, y)=$ $\int_{\left(z_{0}, y_{0}\right)}^{(z, y)}(\langle w\rangle d y-\langle v\rangle d z)$, in a $z-y$ plane. This definition was also used by Ruben and Khosla [63] and Ghia et al. [64] to identify vortex centre in the lid-driven cavity problem. The streamlines around the TLV as well as the vortex centre are shown in Figure 11. 
(a)

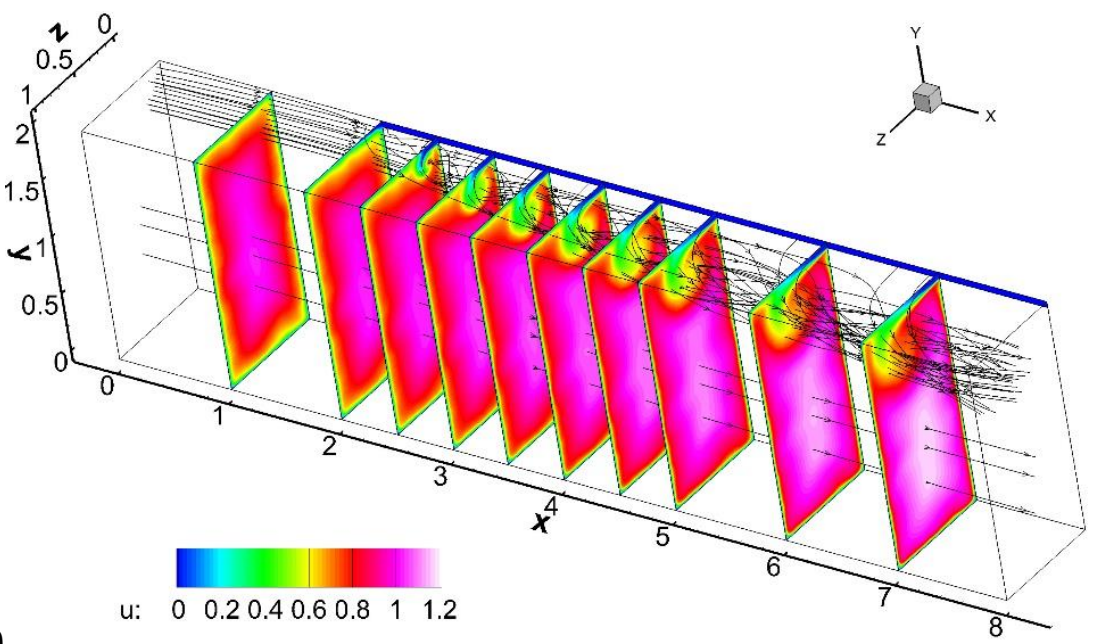

(b)

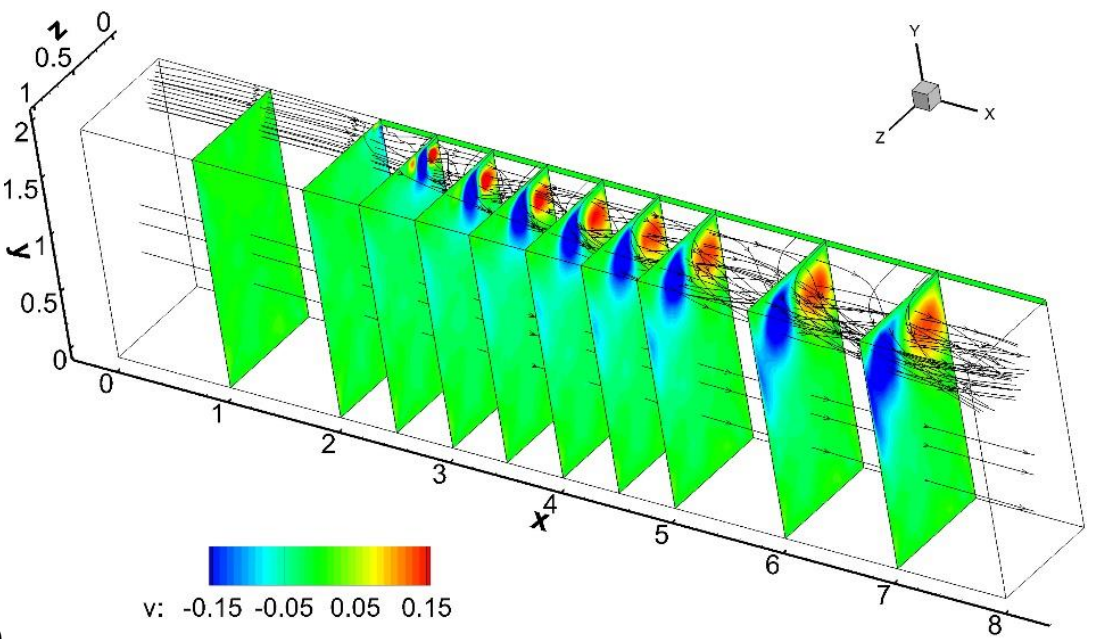

(c)

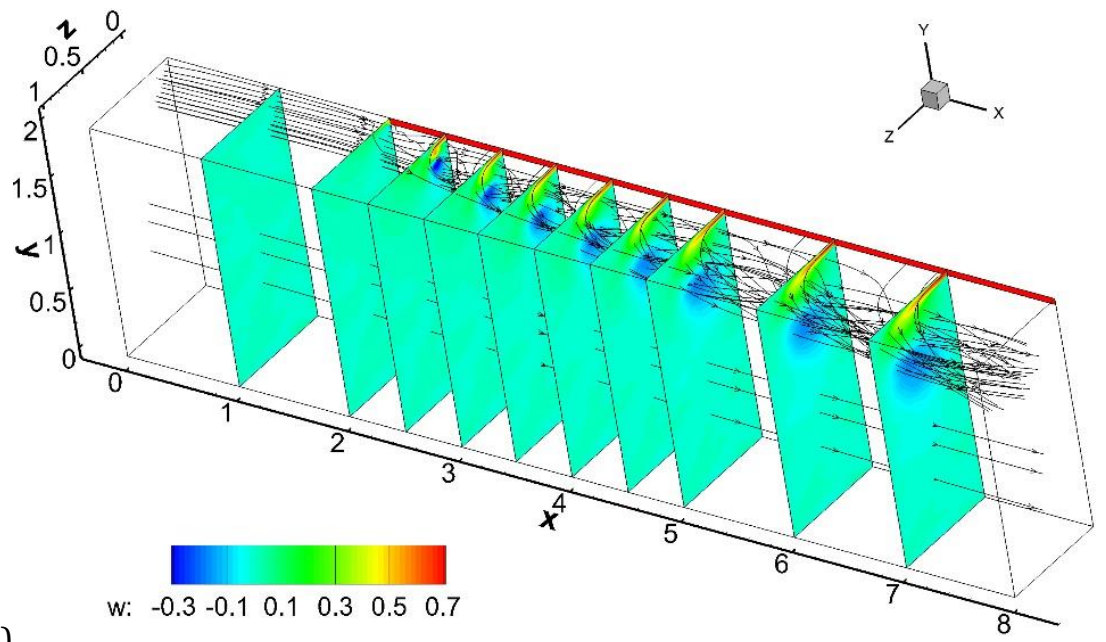

Figure 10. Mean streamwise velocity $\langle u\rangle(a)$, mean y-direction velocity $\langle v\rangle$ (b) and mean z-direction velocity $\langle w\rangle$ (c) on the selected z-y slices along with mean streamlines. 


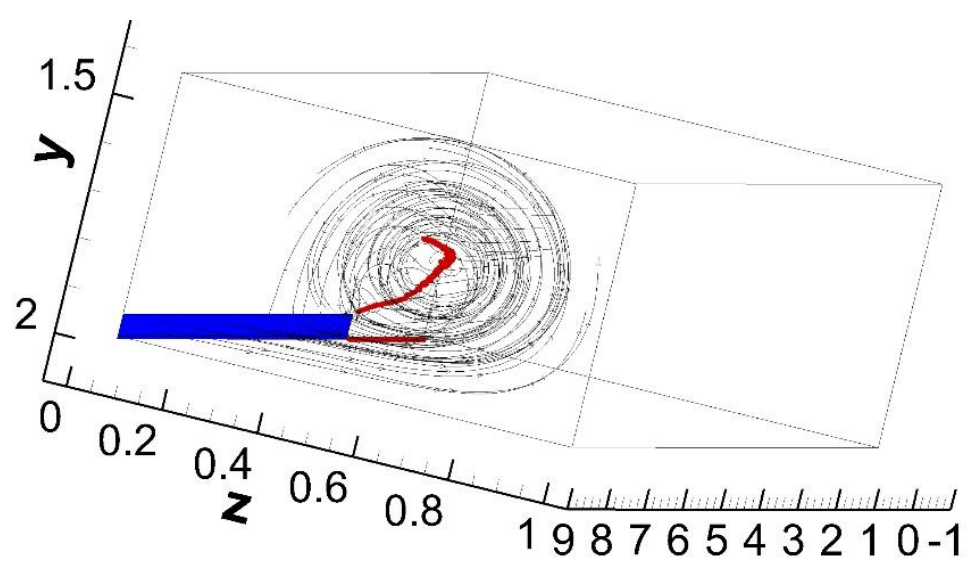

Figure 11. The streamlines and the centre of the TLV (marked as red dots).

The TLV flow structure is further studied by drawing streamlines based on the 2D velocity vector $(\langle w\rangle,\langle v\rangle)$ in the three successive $z-y$ planes at $x=2.5,4.5$ and 7.0, respectively, as shown in Figure 12 . Four vortex cores can be identified at all the three slices. They are categorised as the main TLV, two induced vortex (IV1 and IV2) and one corner vortex (CV). IV1 was also previously observed in a linear cascade ${ }^{[23]}$ and a NACA0009 airfoil with a tip clearance [65,66], and reported as the end-wall boundary layer separation ${ }^{[15] . ~ C V ~ a p p e a r s ~ a t ~ t h e ~ c o r n e r ~}$ is simply because of the existence of the $z_{\max }$ wall. IV2 beneath the slit is induced by the interactions of the TLV and the sidewall, which has not found to be reported in a cascade configuration. The mean streamwise vorticity, $\left\langle\omega_{x}\right\rangle$, at the three selected $z-y$ sections are shown in Figure 13, in which all the three figures present similar patterns of $\left\langle\omega_{x}\right\rangle$. The two legs with high negative and positive $\left\langle\omega_{x}\right\rangle$ are due to the jet/wall and the jet/mainstream interactions respectively. The large area of positive $\left\langle\omega_{x}\right\rangle$ is due to the roll-up of the TLV. At $x=2.5$, the vorticity is highly concentrated with a clear boundary from the main stream, which is consistent with the patterns of the instantaneous streamwise vorticity seen in Figure 6 (a). This indicates highly concentrated vorticity produced at the position. Downstream, the two legs penetrate deeper towards the $+\mathrm{z}$ direction and the vorticity scatters over a larger area. Combing Figure 6 (b), it can be confirmed that the large area of the mean vorticity in Figure 13 (c) is contributed by many small-scale vortices. 
(a)

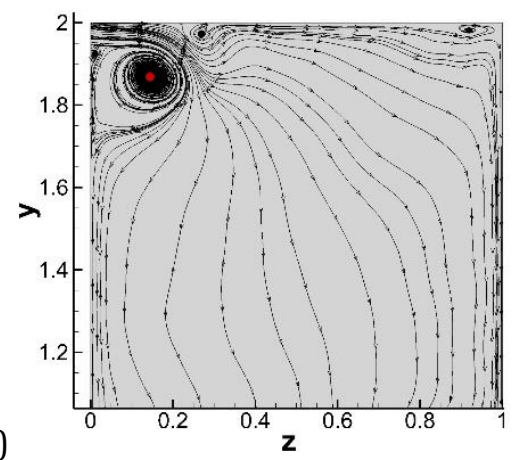

(b)

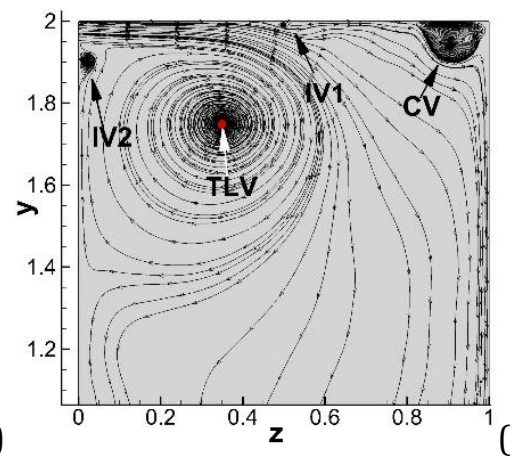

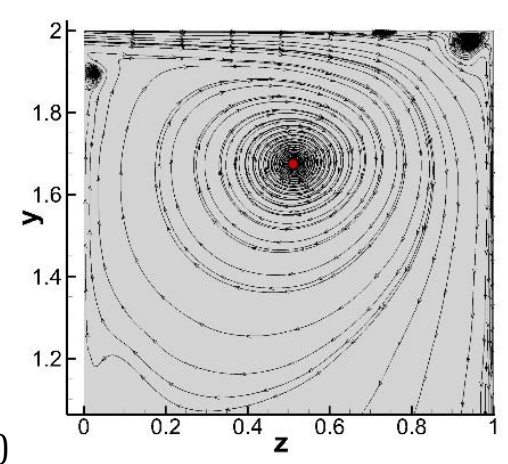

Figure 12. The 2D streamlines $(w, v)$ on three selected $z-y$ planes at $(a): x=2.5,(b) x=4.5$ and $(c) x=7$. The TLV centres are marked as red spots.

(a)

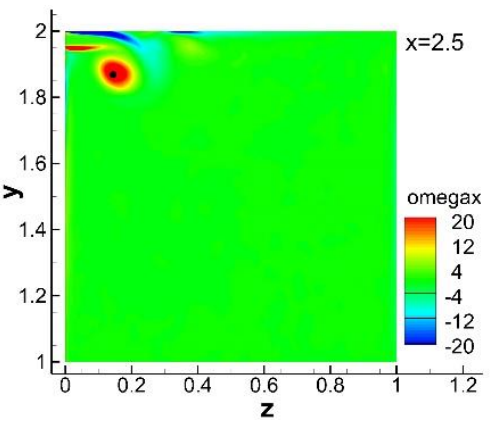

(b)

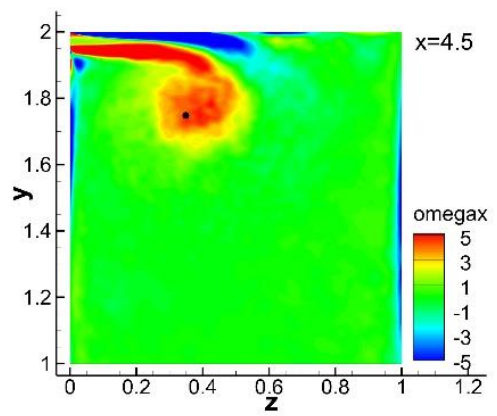

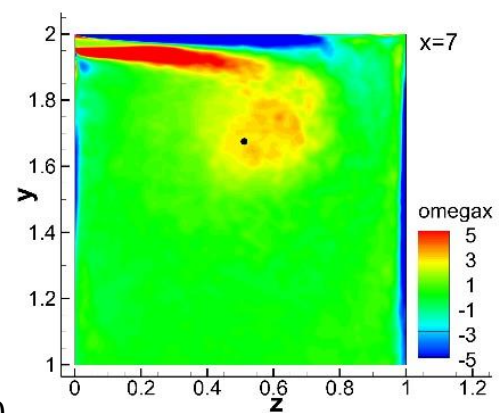

Figure 13. Mean streamwise vorticity at (a): $x=2.5$, (b) $x=4.5$ and (c) $x=7$. The TLV centres are marked as black spots.

\subsection{Turbulence Kinetic Energy}

The turbulence kinetic energy (TKE) defined as, $\mathrm{k}=\frac{1}{2}\left(\left\langle u^{\prime \prime} u^{\prime \prime}\right\rangle+\left\langle v^{\prime \prime} v^{\prime \prime}\right\rangle+\left\langle w^{\prime \prime} w^{\prime \prime}\right\rangle\right)$, at 10 selected slices $(x=1.0,2.0,2.5,3.0,3.5,4.0,4.5$ 5.0, 6.0 and 7.0$)$ is shown in Figure 14, from which we can see a relatively high level of TKE within the TLV region and the diffusion of high TKE area downstream. Prior to the breakup of the TLV (i.e. $x=2.5$ ), TKE is characterised with the two separated peaks: the central TKE peak (CTP) at the vortex core and the peripheral peak surrounding the core on the right side of the vortex, producing a " لـ" shape pattern, which is similar to the PIV measurements in an axial waterjet pump [15,16]. For TLV in the axial waterjet pump, the TKE was observed to have three peaks, respectively within the vorticity sheet connecting the slit, near the TLV centre, and at the interface between positive and negative vorticity layers, prior to the breakup. The high TKE on the right side of TLV is clearly due to the production in the shear-layer between the vortex and the mainstream. During the early stage of TLV evolution, the TKE on the right-side of the shear-layer is increased. After the breakup of TLV, it is gradually damped out, and the TKE pattern changes accordingly. The CTP disappears after the breakup of TLV, and a new high TKE region close to the top wall is formed and strengthened downstream. Therefore, TKE evolves into a " 7 " pattern from $x=4$ to $x=6$ in Figure 14. With the 
further decay of the shear-layer and the deeper penetration of the wall-jet, the TKE becomes a "—" shape pattern at $x=7$. By plotting TKE and mean vorticity components at $x=2.66$ in Figure 15 , where the breakup of TKE happens, we can see the TKE near the edge of the TLV is mainly contributed by $\left\langle\omega_{y}\right\rangle$ and $\left\langle\omega_{z}\right\rangle$, which stands for the shear between low-speed fluids inside of the TLV and the mainstream. The CTP corresponds to the highly concentrated $\left\langle\omega_{x}\right\rangle$ around the TLV core, which means it is mainly contributed from the flow motions in the $z-y$ plane. The TKE and the vorticity distributions at the $x=4.5$ slice are shown in Figure 17, from which a clear " $\urcorner$ " shape of the TKE is observed. The highly compact streamwise vorticity spreads onto a larger area after the breakup of the TLV. Both $\left\langle\omega_{y}\right\rangle$ and $\left\langle\omega_{z}\right\rangle$ present thicker and weaker shear-layers than those at the $x=$ 2.66 section, indicating the diffusion and the dissipation of the shear-layer. The high TKE layer close to the top wall is clearly attributed by both $\left\langle\omega_{x}\right\rangle$ and $\left\langle\omega_{z}\right\rangle$, which is due to the penetration of the wall-jet and the mainstream boundary layer, respectively.

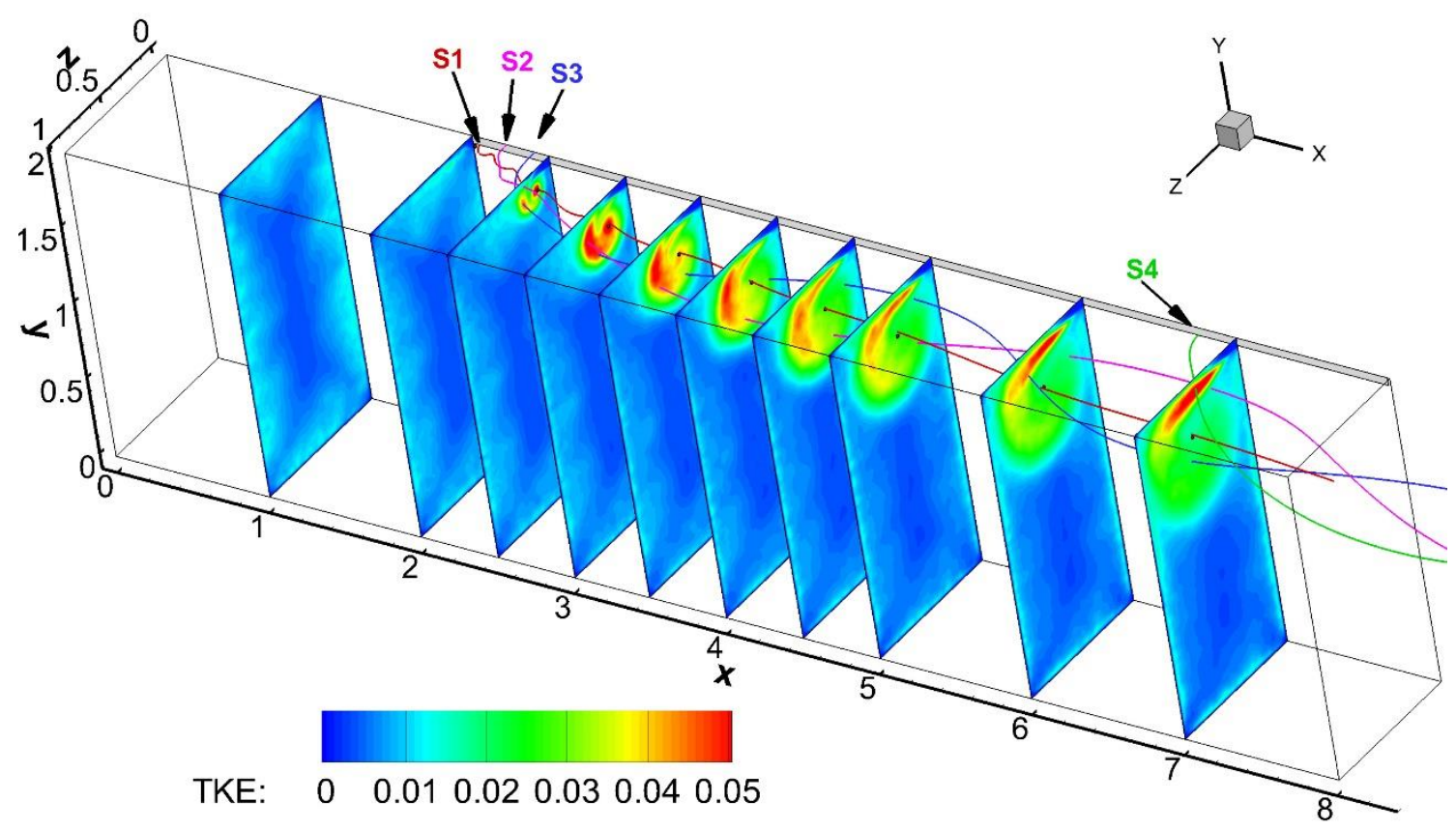

Figure 14. Turbulence kinetic energy on the selected z-y slices. Four mean streamlines are shown, of which S1 passes the TLV centre, S2 and S3 pass the TKE peaks on the TLV right side, S4 passes the TKE peak close to the top wall on the $x=7$ slice. The black spot on each slice marks the TLV centre location. 
(a)

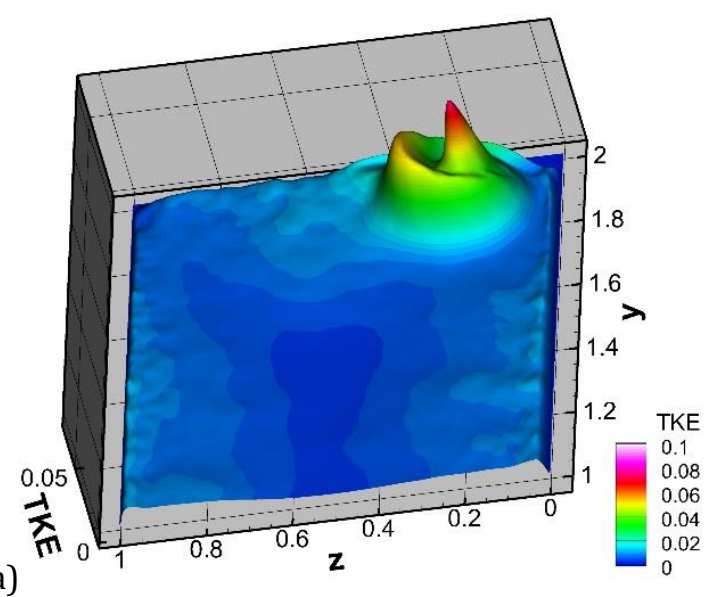

(c)

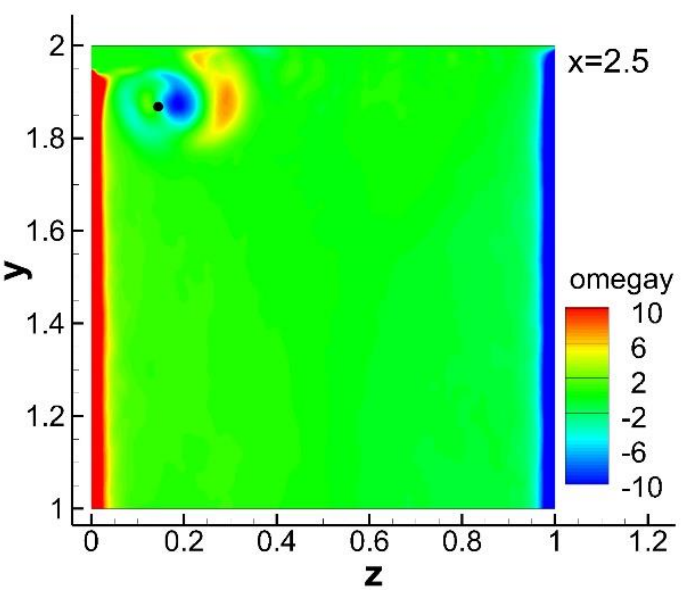

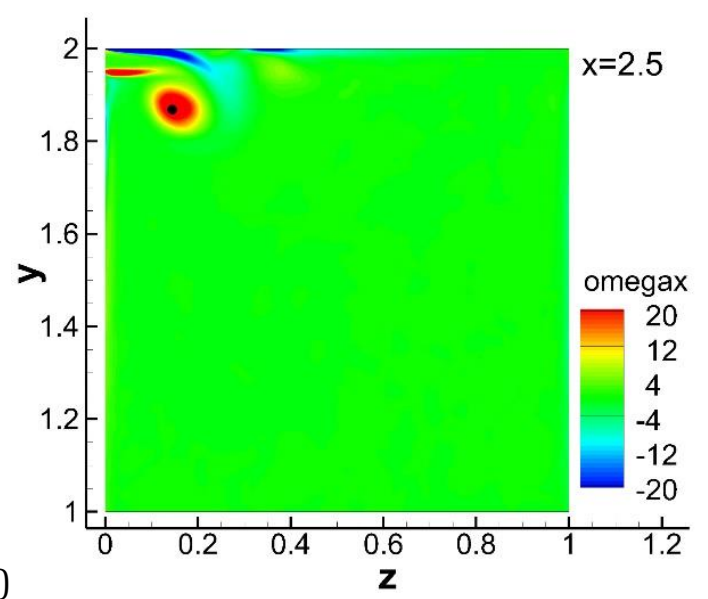

(d)

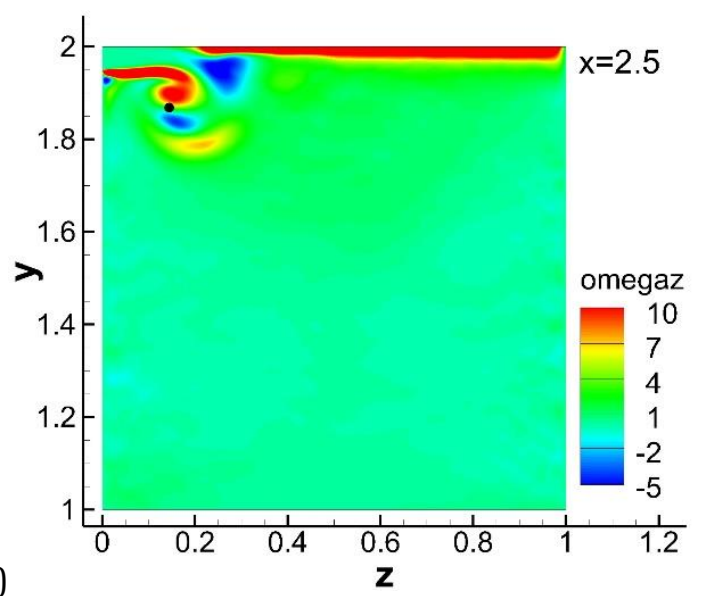

Figure 15. Turbulence kinetic energy and mean vorticity components at $x=2.66$. (a): TKE, (b): $\left\langle\omega_{x}\right\rangle,(c)$ : $\left\langle\omega_{y}\right\rangle,(d):\left\langle\omega_{z}\right\rangle$.

(a)

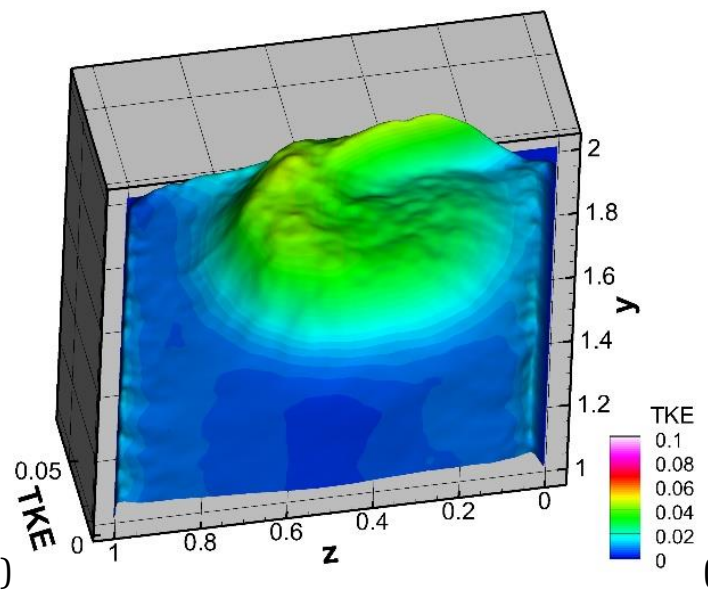

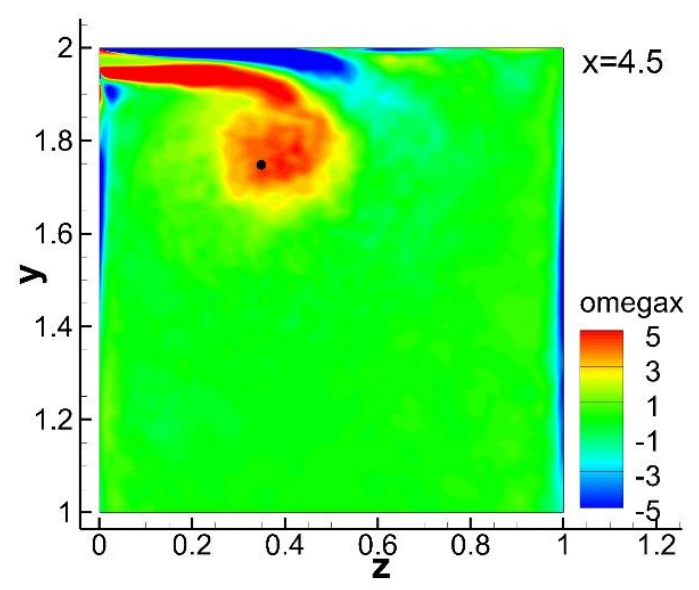


(c)

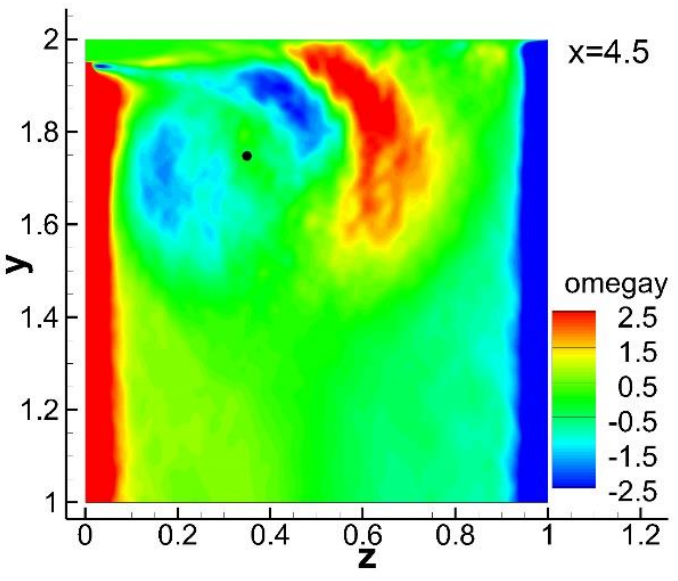

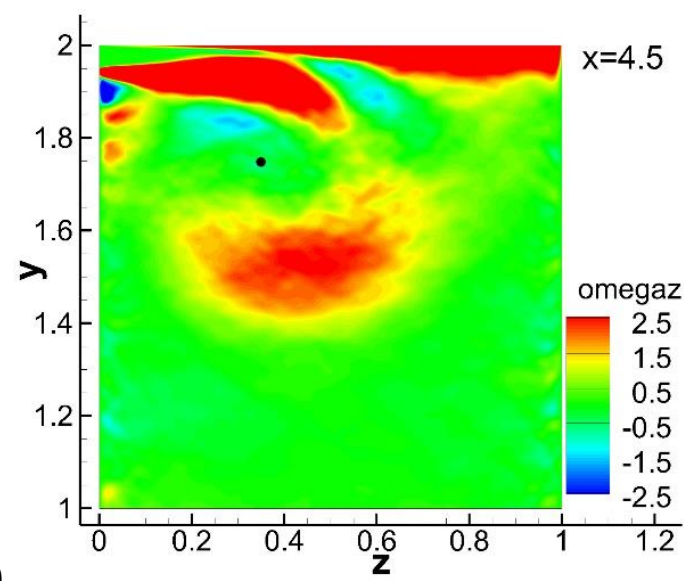

(d)

Figure 16. Turbulence kinetic energy and mean vorticity components at $x=4.5$. (a): TKE, (b): $\left\langle\omega_{x}\right\rangle,(c)$ : $\left\langle\omega_{y}\right\rangle,(d):\left\langle\omega_{z}\right\rangle$. The block spot marks the TLV core.

The TKE profile along the TLV central line is shown in Figure 17, in which the TKE at the vortex centre is rapidly increased, and then decays after its peak location at $x=2.66$. The TKE peak location in Figure 17 is consistent with the TLV breakup location observed in Figure 7, confirming the connection of the disappearance of CTP with the TLV breakup. Comparing the three components of Reynolds stress along the TLV centre line, $\left\langle v^{\prime \prime} v^{\prime \prime}\right\rangle$ is the main contributor in prior of the breakup, producing an anisotropic status of Reynolds stress. After the TKE peak, Reynolds stress at the TLV centre undergoes a recovery process towards an isotropic state.

Miorini et al. ${ }^{[15]}$ attributed the CTP generation mechanism to two possible reasons, the out-of-plane contributors and the transport terms in the TKE equation, which could not be studied in detail due to the limitation of their experimental research. To further explore the mechanism of the production of high TKE at the TLV core, four mean streamlines are extracted in the present study, as presented in Figure 14. The streamwise S1 is selected as the streamline passing through the TLV centre at $x=2.5$. S2 is selected as the one through the TKE peak on the right side of the vortex at $x=2.5$. S3 is selected as the one through the TKE peak at $x=3$, and S4 selected as the one through the TKE peak at $x=7$. 


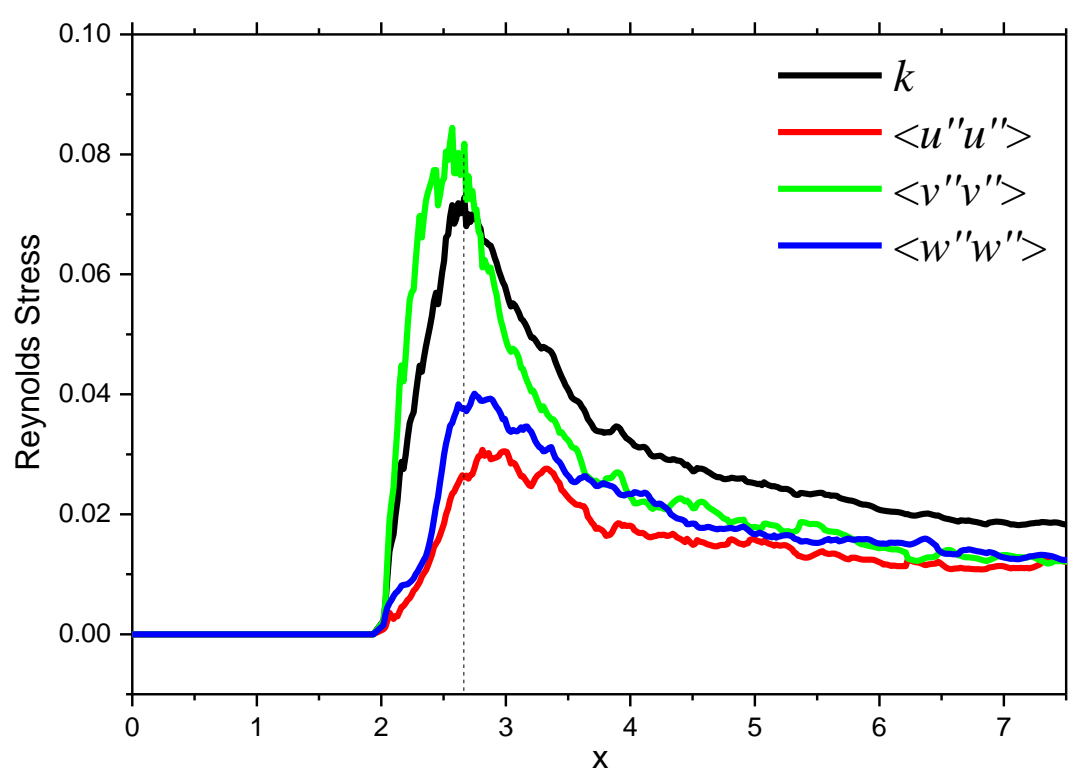

Figure 17. Evolution of Reynolds stress $\left\langle u_{i}^{\prime \prime} u_{i}^{\prime \prime}\right\rangle$ and TKE at the TLV centre.

Note that the streamline S1 undergoes a highly spiral meandering during the early stage of the TLV evolution, which can be seen more clearly in the zoomed view in Figure 18 (a). This kind of mean flow behaviour could have a major contribution to the CTP, and it also explains $\left\langle v^{\prime \prime} v^{\prime \prime}\right\rangle$ being the dominant TKE component. The compactly spiral meandering of the vortex filament interacts with the large velocity gradient compound $\partial\langle v\rangle / \partial\langle z\rangle$ on the $z-y$ plane, creating large velocity fluctuations $v^{\prime \prime}$ around the TLV core. This agrees with the conclusion of "meandering of vortex filaments dominates the TKE" drawn by Miorini et al. [15]. This process can be further demonstrated by observing the streamline S1 and the mean velocity $\langle v\rangle$ during the early stage of TLV generation as shown in Figure 18 (b). The streamline S1 'jumps' between positive and negative regions within a compact space, which exchanges momentum between two regions and generates strong $\left\langle v^{\prime \prime} v^{\prime \prime}\right\rangle$ around the TLV core. This kind of momentum exchange is basically a mean flow mixing process. After the breakup of the TLV, the meandering of streamline becomes much smoother, and therefore the central TKE 'hot' spot disappears. The helical motions of S2 and S3 are less violent than S1, which means they have less momentum exchanging effect, and the high TKE on the right side of the TLV is mainly due to local shear production. 
(a)

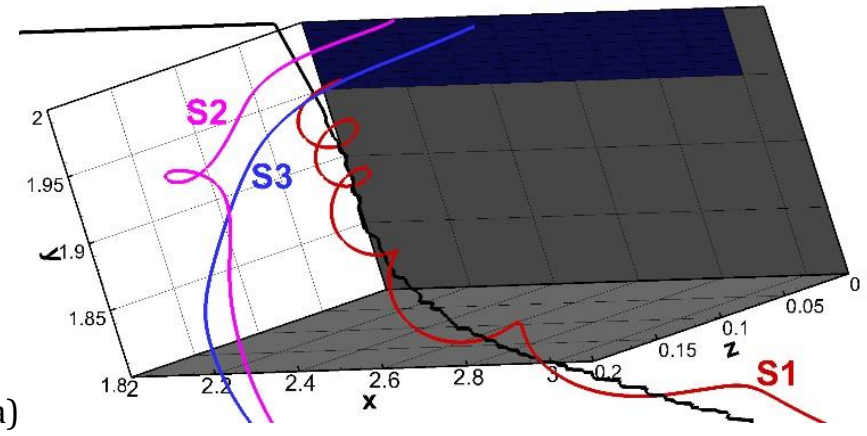

(b)

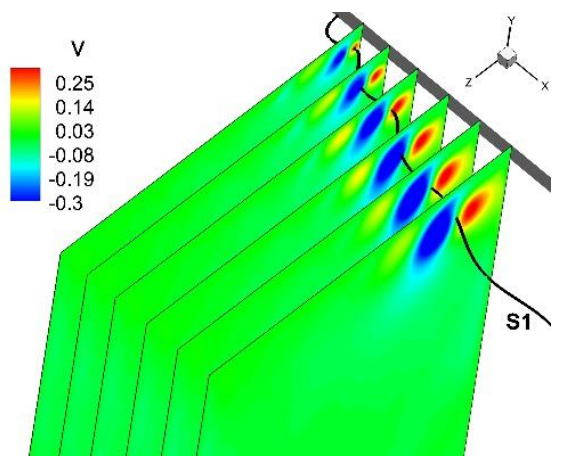

Figure 18. (a): Streamlines S1 (red), S2(purple) and S3 (blue) and TLV centre trace lines (black) in the zoomed view. (b): Streamline $S 1$ and distribution of mean velocity $\langle v\rangle$.

\subsection{TKE Transport Characteristic}

The TKE transport equation or the budget equation,

$$
\begin{aligned}
& \frac{\partial \bar{\rho} k}{\partial t}=\underbrace{-\frac{\partial \bar{\rho} k\left\langle u_{j}\right\rangle}{\partial x_{j}}}_{C} \underbrace{-\frac{\partial}{\partial x_{j}}\left[\bar{\rho}\left\langle u_{j}^{\prime \prime} k\right\rangle+\overline{p^{\prime} u_{j}^{\prime \prime}}\right]}_{T} \underbrace{+\frac{\partial}{\partial x_{j}} \overline{u_{l}^{\prime \prime} \sigma_{\jmath l}\left(u^{\prime \prime}\right)}}_{V} \underbrace{-\bar{\rho}\left\langle u_{i}^{\prime \prime} u_{j}^{\prime \prime}\right\rangle \frac{\partial\left\langle u_{i}\right\rangle}{\partial x_{j}}}_{P} \underbrace{-\sigma_{\jmath l}(\langle u\rangle) \frac{\partial u_{l}^{\prime \prime}}{\partial x_{j}}}_{\varepsilon} \\
& \underbrace{+\underbrace{\prime} \frac{\partial u_{l}^{\prime \prime}}{\partial x_{\imath}}-\overline{u_{\jmath}^{\prime \prime}} \frac{\partial \bar{p}}{\partial x_{j}}+\overline{u_{\imath}^{\prime \prime}} \frac{\partial \sigma_{j i}(\langle u\rangle)}{\partial x_{j}}}_{K}
\end{aligned}
$$

is further analysed to study turbulence mechanism in terms of TKE generation. Each term in Eq. (7) represent a specific dynamic process of TKE behaviour. The turbulence kinetic energy budget terms are classified as the convection term, $C$, the turbulence transport term, $T$, the viscous diffusion term, $V$, the production term, $P$, the dissipation term, $\varepsilon$, and the compressibility term, $K$. The overbar, - , stands the Reynolds-averaging operator, and the superscript, ', represents the fluctuation from a Reynolds-averaged variable. The viscous stress tensor, $\sigma_{j i}(\langle u\rangle)$ and $\sigma_{j i}\left(u^{\prime \prime}\right)$, are respectively calculated with mean and fluctuation velocities.

The distributions of the TKE budget terms at the $x=2.66$ and $x=4.5$ sections are shown in Figure 19 and Figure 20 , respectively. At $x=2.66$, the production rate is positive and high in the shear-layer, but low and even negative at the centre, indicating the different TKE production mechanisms in the vortex core and the shear-layer. It is interesting to note that the TKE has a large peak in the vortex core region (shown in Figure 15 a), but the TKE production presents a negative value. This contradiction is also observed in the measurement of Miorini et al. [15]. The negative production indicates that the turbulence is in a non-equilibrium state, e.g. there is a difference between the change of the mean strain rate and the response of the Reynolds stress [17]. The dissipation rate in the TLV region is at an order of magnitude smaller than the production. The turbulence 
transport term, $T$, plays a significant role in the re-distribution of turbulence kinetic energy. It has a similar magnitude as the production term and transports TKE from the shear-layer towards the central and the outer regions. The convection term, $C$, also has a comparable order of magnitude as $P$ and $T$, and it is negative in the shear-layer, but positive at the vortex centre. This observation is consistent with the analysis of the highly spiral mean streamline S1 and its mixing effect. Therefore, it can be concluded that the CTP is mainly due to the convection effect through the highly spiral motion of the mean flow. The viscous diffusion term, $V$, balances the TKE through the viscous effect. But its magnitude is an order smaller than $P, T$, and $C$, thus the effect may not be significant. The compressibility effect is also negligible since the flow is weakly-compressible.

(a)

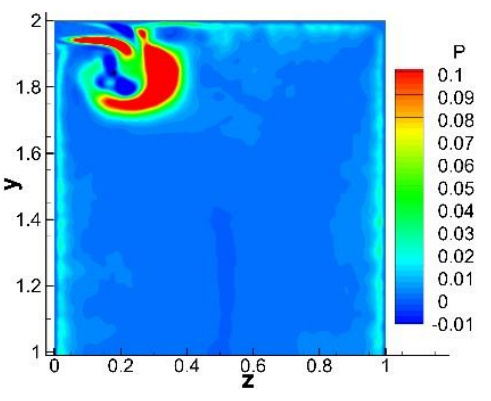

(b)

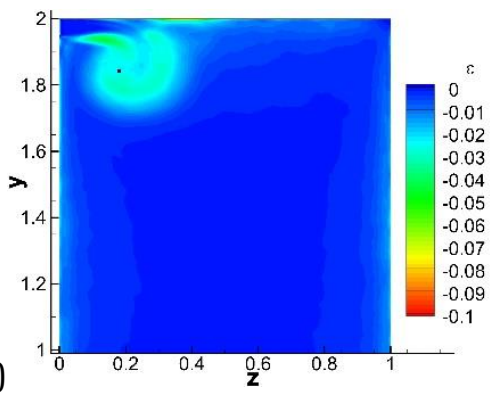

(c)

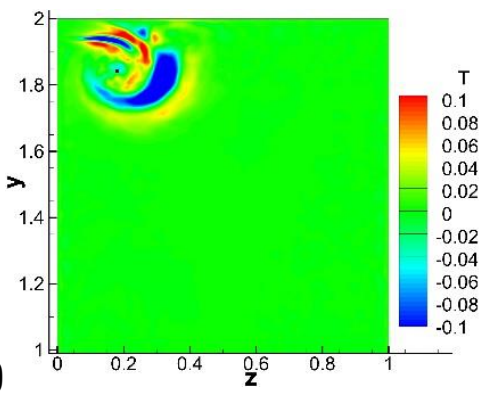

(d)

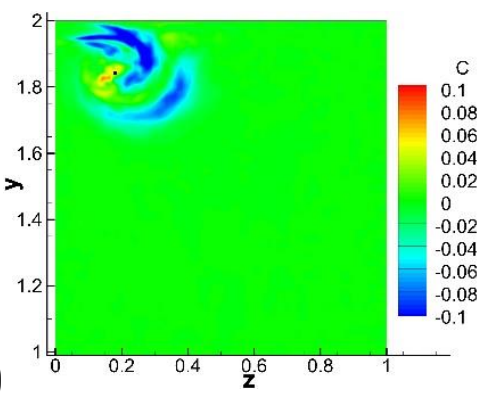

(e)

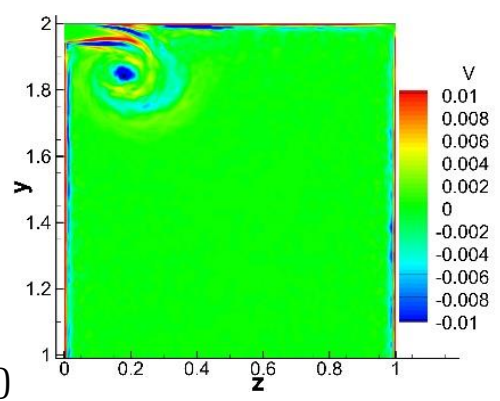

Figure 19. TKE budget terms at $x=2.66$. (a): $P,(b): \varepsilon,(c): T,(d): C,(e)$ : $V$. The black spot marks the TLV core.

At the $x=4.5$ section, where the TLV has broken up, the budget presents a shear dominating TKE transport characteristic. The production term has high values in the two shear-layers, which leads to high values of TKE (as shown in Figure 16 a) in the corresponding regions. The dissipation term is also high in the shear-layers. The turbulent transport term is responsible for the redistribution of TKE in the $z-y$ section, i.e. transport of TKE from high production regions to the surrounding low production regions. The convection term mainly transports turbulent energy along the streamwise direction. At the TLV core, where the shear strength is relatively weak, all terms are not significant. 
(a)

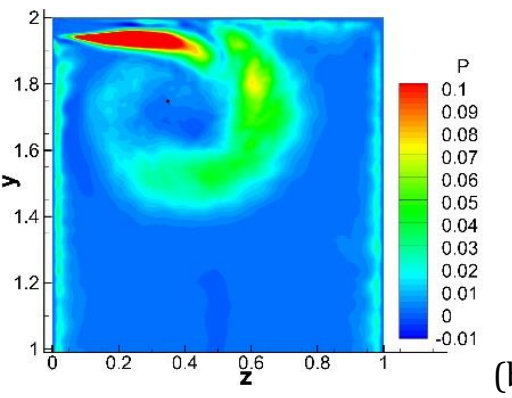

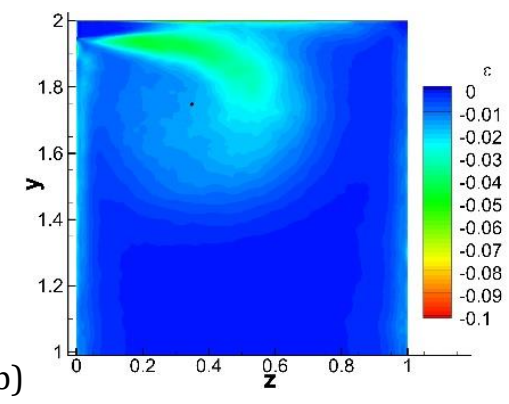

(c)

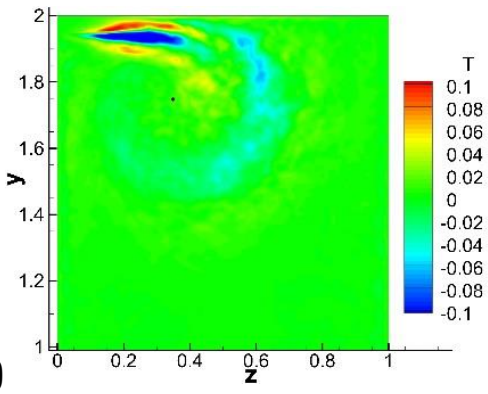

(d)

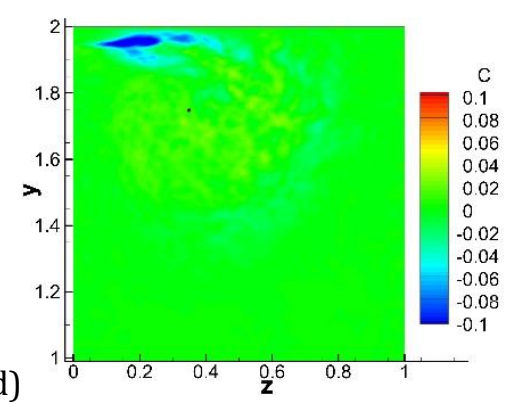

(e)

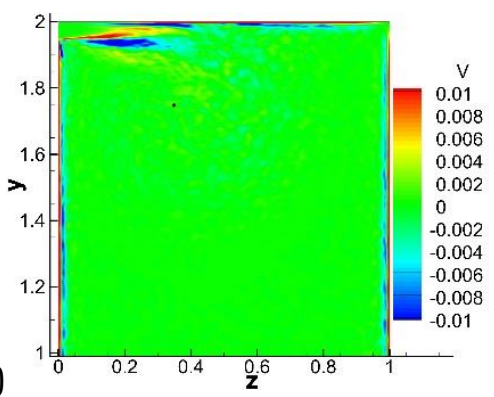

Figure 20. TKE budget terms at $x=4.5$. (a): $P,(b): \varepsilon$, (c): $T$, (d): $C$, (e): $V$. The black spot marks the TLV core.

\section{Conclusions}

A flow model, which consists of a planar duct with a longitudinal slit, is studied using DNS at $M a=0.5, R e=$ 10,000 , to study the tip-leakage vortex flow. The results are validated by comparing the profiles of velocity and Reynolds stress in the inlet plane with the DNS of channel flow. This is followed by the study of instantaneous and mean flow fields, and then the discussion of detailed turbulence structures and vortex behaviours. Turbulence kinetic energy budget equation is also analysed.

It is found that the interaction between the main passage flow in the duct and the jet flow from the slit generates a streamwise vortex, resembling the tip-leakage vortex. A low-speed flow region is formed within the TLV area, leading to a blockage effect, which is often observed in turbomachinery. The vortical flow in the passage is found strongly three-dimensional and complicated. Major features of the TLV in turbomachinery are identified by the current model, including the main TLV, induced vortices besides the TLV, and the corner vortex. The vorticity of the TLV is highly concentrated during its initial stage, and after a critical point, it breaks up into a package of many small-scale vortical structures.

The turbulence in the TLV area is energetic, which can be observed in the velocity fluctuations and the TKE distribution. With the development of the TLV, the evolution of TKE can be divided into three phases. The first 
is the formation phase, which is from the formation of TLV to the break-up point. During the formation phase, the TKE presents two separated peaks, one at the centre of the TLV and the other in the outer layer of the vortex. The second phase is called the break-up phase, in which the TLV breaks up into many small vortices. The central peak of TKE disappears, while the vortical region expands. In the third phase, namely the diffusion phase, the turbulence within the vortical area is relatively weak, and strong turbulence is observed in the jet shear-layer.

The analysis of mean streamline shows that a mean flow mixing process happens at the vortex centre in the formation phase, which exchanges the fluid momentum between the positive and the negative y-velocity regions and causes a high level of velocity fluctuations. This process is primarily a result of the spiral meandering of the vortex filament. The further investigation of the TKE budget equation shows that the TKE is mainly produced in the shear-layer on the outer part of the TLV, then transported towards the TLV centre by the turbulent transport. Meanwhile, the central high TKE region is contributed by the convection effect, as a result of the mixing effect of the highly spiral mean flow.

\section{Acknowledgement}

The project is supported by the Engineering and Physical Science Research Council (EPSRC) through the UK Consortium on Turbulent Reacting Flows (Grant numbers EP/R029369/1 and EP/K024574/1) and the UK Turbulence Consortium (Grant number EP/L000261/1), and the National Natural Science Foundation of China (51420105008, 51676007, 51790513 and 51976006). The simulation was conducted on the UK HighPerformance Computing Service ARCHER.

\section{References}

[1] J. D. Denton, Some limitations of turbomachinery CFD, ASME Turbo Expo 2010: Power for Land, Sea, and Air, American Society of Mechanical Engineers, 2010: 735-745.

[2] T. C. Booth, P. R. Dodge, and H. K. Hepworth, Rotor-tip leakage part I-basic methodology, Journal of Engineering for Gas Turbines \& Power, 1982, 104(1):154-161.

[3] M. Inoue, M. Kuroumaru, and M. Fukuhara, Behavior of tip leakage flow behind an axial compressor rotor, Journal of Engineering for Gas Turbines and Power, 1986, 108(1): 7-14.

[4] C. T. Hsiao, and G. L. Chahine, Scaling of tip vortex cavitation inception noise with a bubble dynamics model accounting for nuclei size distribution, Journal of Fluids Engineering, 2005, 127(1): 55-65.

[5] J. J. Adamczyk, M. L. Celestina, and E. M. Greitzer, The role of tip clearance in high-speed fan stall, Journal of Turbomachinery, 1993, 115(1):28-39.

[6] C. S. Tan, I. Day, S. Morris, and A. Wadia, Spike-type compressor stall inception, detection, and control, Annual Review of Fluid Mechanics, 2010, 42: 275-300. 
[7] Z. Xie, Y. Liu, X Liu, D. Sun, L. Lu, and X. Sun, Computational model for stall inception and nonlinear evolution in axial flow compressors, Journal of Propulsion and Power, 2017, 34(3): 720-729.

[8] J. Wells, A. Salem-Said, and S. A. Ragab, Effects of turbulence modeling on RANS simulations of tip vortices, 48th AIAA Aerospace Sciences Meeting Including the New Horizons Forum and Aerospace Exposition, 2010: 1104.

[9] M. Furukawa, M. Inoue, K. Saiki, and K. Yamada, The role of tip leakage vortex breakdown in compressor rotor aerodynamics, Journal of Turbomachinery, 1999, 121(3): 469-480.

[10] Y. Liu, X. Yu, and B. Liu, Turbulence models assessment for large-scale tip vortices in an axial compressor rotor, Journal of Propulsion and Power, 2008, 24(1): 15-25.

[11] D. C. Wilcox, Turbulence modeling for CFD, La Canada, CA: DCW industries, 1998.

[12] Y. Gao, Y. Liu, L. Zhong, J. Hou, and L. Lu, Study of the standard k- $\varepsilon$ model for tip leakage flow in an axial compressor rotor, International Journal of Turbo \& Jet-Engines, 2016, 33(4): 353-36.

[13] X. Yu, and B. Liu, Stereoscopic PIV measurement of unsteady flows in an axial compressor stage, Experimental Thermal and Fluid Science, 2007, 31(8): 1049-1060.

[14] H. Du, X. Yu, Z. Zhang, and B. Liu, Relationship between the flow blockage of tip leakage vortex and its evolutionary procedures inside the rotor passage of a subsonic axial compressor, Journal of Thermal Science, 2013, 22(6): 522-531.

[15] R. L. Miorini, H. Wu, and J Katz, The internal structure of the tip leakage vortex within the rotor of an axial waterjet pump, Journal of Turbomachinery, 2012, 134(3): 031018.

[16] H. Wu, R. L. Miorini, D. Tan, and J. Katz, Turbulence within the tip-leakage vortex of an axial waterjet pump, AIAA Journal, 2012, 50(11): 2574-2587.

[17] Y. Li, C. Huang, and J. Katz. Measurements and characterization of turbulence in the tip region of an axial compressor rotor, Journal of Turbomachinery, 2017, 139(12): 121003.

[18] J. Decaix, G. Balarac, M. Dreyer, M. Farhat, and C. Münch, RANS and LES computations of the tip-leakage vortex for different gap widths, Journal of Turbulence, 2015, 16(4): 309-341.

[19] Y. Liu, L. Lu, L. Fang, F. Gao, Modification of Spalart-Allmaras model with consideration of turbulence energy backscatter using velocity helicity, Physics Letters A, 2011, 375(24): 2377-2381.

[20] K. B. Lee, M. Wilson, and M. Vahdati, Validation of a numerical model for predicting stalled flows in a lowspeed fan-part I: Modification of spalart-allmaras turbulence model, Journal of Turbomachinery, 2018, 140(5):051008.

[21] D. You, M. Wang, P. Moin, and R. Mittal, Effects of tip-gap size on the tip-leakage flow in a turbomachinery cascade, Physics of Fluids, 2006, 18(10): 105102.

[22] D. You, M. Wang, P. Moin, and R. Mittal, Large-eddy simulation analysis of mechanisms for viscous losses in a turbomachinery tip-clearance flow, Journal of Fluid Mechanics, 2007, 586: 177-204.

[23] D. You, M. Wang, P. Moin, and R. Mittal, Vortex dynamics and low-pressure fluctuations in the tip-clearance flow, Journal of Fluids Engineering, 2007, 129(8): 1002-1014.

[24] A. Pogorelov, M. Meinke, and W. Schröder, Cut-cell method based large-eddy simulation of tip-leakage flow. Physics of Fluids, 2015, 27(7): 075106.

[25] S. M. A. Moghadam, M. Meinke, and W. Schröder, Analysis of tip-leakage flow in an axial fan at varying tipgap sizes and operating conditions, Computer \& Fluids, 2019, 183(15): 107-129.

[26] J. Boudet, A. Cahuzac, P. Kausche, and M. C. Jacob, Zonal large-eddy simulation of a fan tip-clearance flow, with evidence of vortex wandering, Journal of Turbomachinery, 2015, 137(6): 061001.

[27] J. Boudet, J. Caro, B. Li, E. Jondeau, and M. C. Jacob, Zonal large-eddy simulation of a tip leakage flow, International Journal of Aeroacoustics, 2016, 15(6-7): 646-661.

[28] Y. Liu, L. Zhong, and L. Lu, Comparison of DDES and URANS for unsteady tip leakage flow in an axial compressor rotor, Journal of Fluids Engineering, 2019, 141(12): 121405.

[29] X. Su, X. Ren, X. Li, and C. Gu, Unsteadiness of tip leakage flow in the detached-eddy simulation on a transonic rotor with vortex breakdown phenomenon, Energies, 2019, 12(5): 954.

[30] P. Moin, and K. Mahesh, Direct numerical simulation: A tool in turbulence research, Annual Review of Fluid Mechanics, 1998, 30: 539-78.

[31] D. A. Rains, Tip clearance flows in axial flow compressors and pumps, 1954, Ph.D. thesis, California Institute of Technology, Pasadena, CA. 
[32] B. Lakshminarayana, Methods of predicting the tip clearance effects in axial flow turbomachinery, Journal of Basic Engineering, 1970, 92(3): 467-480.

[33] G. T. Chen, E. M. Greitzer, C. S. Tan, and F. E. Marble, Similarity analysis of compressor tip clearance flow structure, Journal of Turbomachinery, 1991, 113(2): 260-269.

[34] A. P. S. Wheeler, and R. D. Sandberg, Direct numerical simulations of a transonic tip flow with free-stream disturbances, asme turbine blade tip symposium, ASME 2013 Turbine Blade Tip Symposium V001T01A002, 2013.

[35] A. P. S. Wheeler, R. D. Sandberg, N. D. Sandham, R. Pichler, V. Michelassi, and G. M. Laskowski, Direct numerical simulations of a high pressure turbine vane, journal of turbomachinery, 2016, 138(7):071003.

[36] A. P. S. Wheeler, and R. D. Sandberg, Numerical investigation of the flow over a model transonic turbine blade tip, Journal of Fluid Mechanics, 2016, 803: 119-143.

[37] I. Z. Naqavi, J. C. Tyacke, P. G. Tucker. Direct numerical simulation of a wall jet: flow physics. Journal of Fluid Mechanics, 2018, 852: 507-542.

[38] A. R. Karagozian, The jet in crossflow, Physics of Fluids, 2014, 26(10): 1-47.

[39] L. Zhu, W. Zhang, J. Kou, et al. Machine learning methods for turbulence modeling in subsonic flows around airfoils. Physics of Fluids, 2019, 31(1): 015105.

[40] Z. Wang, K. Luo, D. Li, et al. Investigations of data-driven closure for subgrid-scale stress in large-eddy simulation. Physics of Fluids, 2018, 30(12): 125101.

[41] Y. Gao, Y. Liu, and L. Lu, Investigation of turbulence characteristics in a tip leakage flow model using largeeddy simulation. ASME paper GT2019-90844.

[42] Y. Gao, and Y. Liu, A flow model for tip leakage flow in turbomachinery using a square duct with a longitudinal slit. Aerospace Science and Technology, 2019: 105460.

[43] J. Fang, Y. Yao, Z. Li and L. Lu, Investigation of low-dissipation monotonicity-preserving scheme for direct numerical simulation of compressible turbulent flows, Computer \& Fluids, 2014, 104: 55-72.

[44] J. Fang, Y. Yao, A.A. Zheltovodov, Z. Li and L. Lu, Direct numerical simulation of supersonic turbulent flows around a tandem expansion-compression corner, Physics of Fluids, 2015, 27, 125104.

[45]Ni, W., Lu, L., Le Ribault, C., and Fang, J., Direct numerical simulation of supersonic turbulent boundary layer with spanwise wall oscillation, Energies, 2016, 9(3): 1-24.

[46] J. Fang, Y. Yao, A. A. Zheltovodov, and L. Lu, Investigation of three-dimensional shock wave/ turbulentboundary- layer interaction initiated by a single fin, AIAA Journal, 2017, 55(2): 509-523.

[47]Ni, W., Lu, L., Fang, J., Moulinec, C., and Yao, Y., Large-scale streamwise vortices in turbulent channel flow induced by active wall actuations, flow, Turbulence and Combustion, 2017, 100(3): 651-673.

[48]Ni, W., Lu, L., Fang, J., Moulinec, C., Emerson, D.R., and Yao, Y., Flow separation control over a rounded ramp with spanwise alternating wall actuation, Physics of Fluids, 2019, 31(1):015101.

[49]Lele, S. K., Compact finite difference schemes with spectral-like resolution, Journal of Computational Physics, 1992, 103: 16-42.

[50] J. Fang, F. Gao, C. Moulinec, and D. R. Emerson, An improved parallel compact scheme for domaindecoupled simulation of turbulence, International Journal of Numerical Method in Fluids, 2019, 90(10): 479-500.

[51] N. D. Sandham, Q. Li, and H. C. Yee, Entropy splitting for high-order numerical simulation of compressible turbulence, Journal of Computational Physics, 2002, 178(2): 307-322.

[52] D. V. Gaitonde, and M. R. Visbal, Pade-type higher-order boundary filters for the Navier-Stokes equations, AIAA Journal, 2000, 38(11): 2103-2112.

[53] S. Gottlieb, and C. W. Shu, Total variation diminishing Runge-Kutta schemes, Mathematics of Computation, 1998, 67(221), 73-85.

[54] P. Sagaut, Theoretical background: Large-eddy simulation, in: Large-eddy simulation for acoustics, 1st ed., 2007, Cambridge University Press: Cambridge, UK. pp. 89-127.

[55] E. Lenormand, P. Sagaut, and L. T. Phuoc, Large eddy simulation of subsonic and supersonic channel flow at moderate Reynolds number, International Journal for Numerical Methods in Fluids, 2000, 32(4): 369406.

[56] J. D. H. Rudy, and J. C. Strikwerda, A nonreflecting outflow boundary condition for subsonic navier-stokes calculations, Journal of Computational Physics, 1980, 36(1): 55-70. 
[57] J. W. Kim, and D. J. Lee, Generalized characteristic boundary conditions for computational aeroacoustics, AIAA Journal, 2000, 38(11): 2040-2049.

[58] P. Orlandi, and S. Pirozzoli, DNS of transitional and turbulent flows in rectangular ducts, Physics of Fluids, 2018, arXiv:1907.07987v1.

[59] R. D. Moser, J. Kim, and N. N. Mansour, Direct numerical simulation of turbulent channel flow up to $\operatorname{Re}_{\tau}=590$, Physics of Fluids, 1999, 11(4): 943-945.

[60] J. Zhou, R. J. Adrian, S. Balachandar, and T. M. Kendall, Mechanisms for generating coherent packets of hairpin vortices in channel flow. Journal of Fluid Mechanics, 1999, 387:353-96.

[61]S. Pirozzoli, M. Bernardini, and F. Grasso, Characterization of coherent vortical structures in a supersonic turbulent boundary layer, Journal of Fluid Mechanics, 2008, 613: 205-231.

[62] S. J. Kline, W. C. Reynolds, and F. A. Schraub, The structure of turbulent boundary layers, Journal of Fluid Mechanics, 1967, 30: 741-773.

[63] S. G. Ruben, and P. K. Khosla, Polynomial interpolation methods for viscous flow calculations, Journal of Computational Physics, 1977, 24: 217-244.

[64] U. Ghia, K. N. Ghia, and C. T. Shin, High-Re solutions for incompressible flow using the Navier-Stokes equations and a multigrid method, Journal of Computational Physics, 1982, 48: 387-411.

[65] M. Dreyer, J. Decaix, C. Münch, and M. Farhat, Mind the gap - a new insight on the tip leakage vortex using stereo PIV, Experiments in Fluids, 2014, 55(11): 1849.

[66] J. Decaix, G. Balarac, M. Dreyer, M. Farhat, and C. Münch, RANS and LES computations of the tipleakage vortex for different gap widths, Journal of Turbulence, 2015, 16(4): 309-341. 


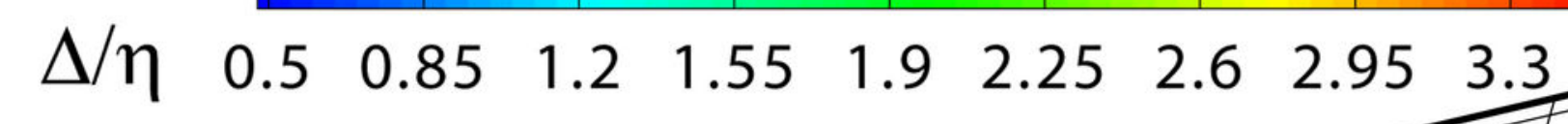




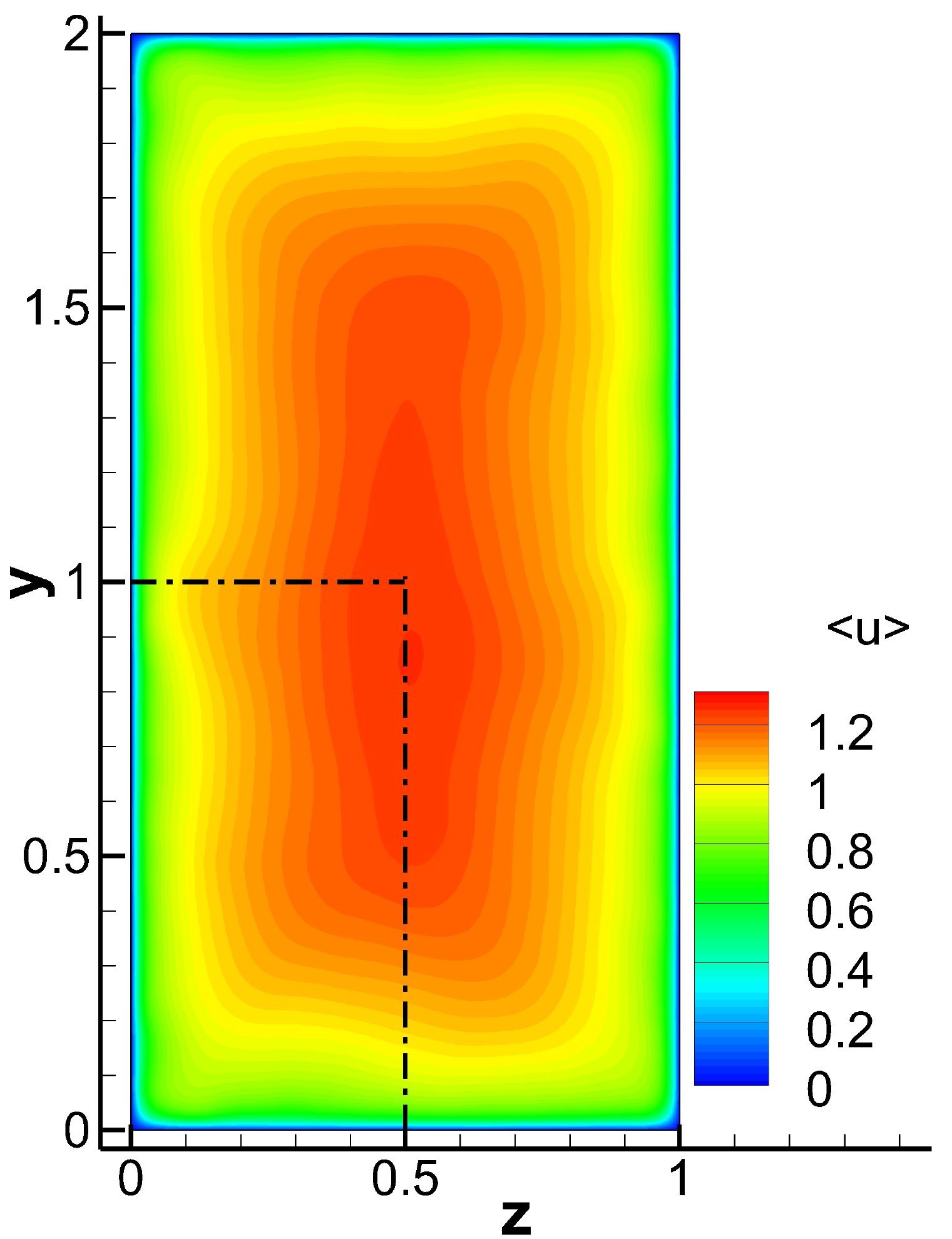




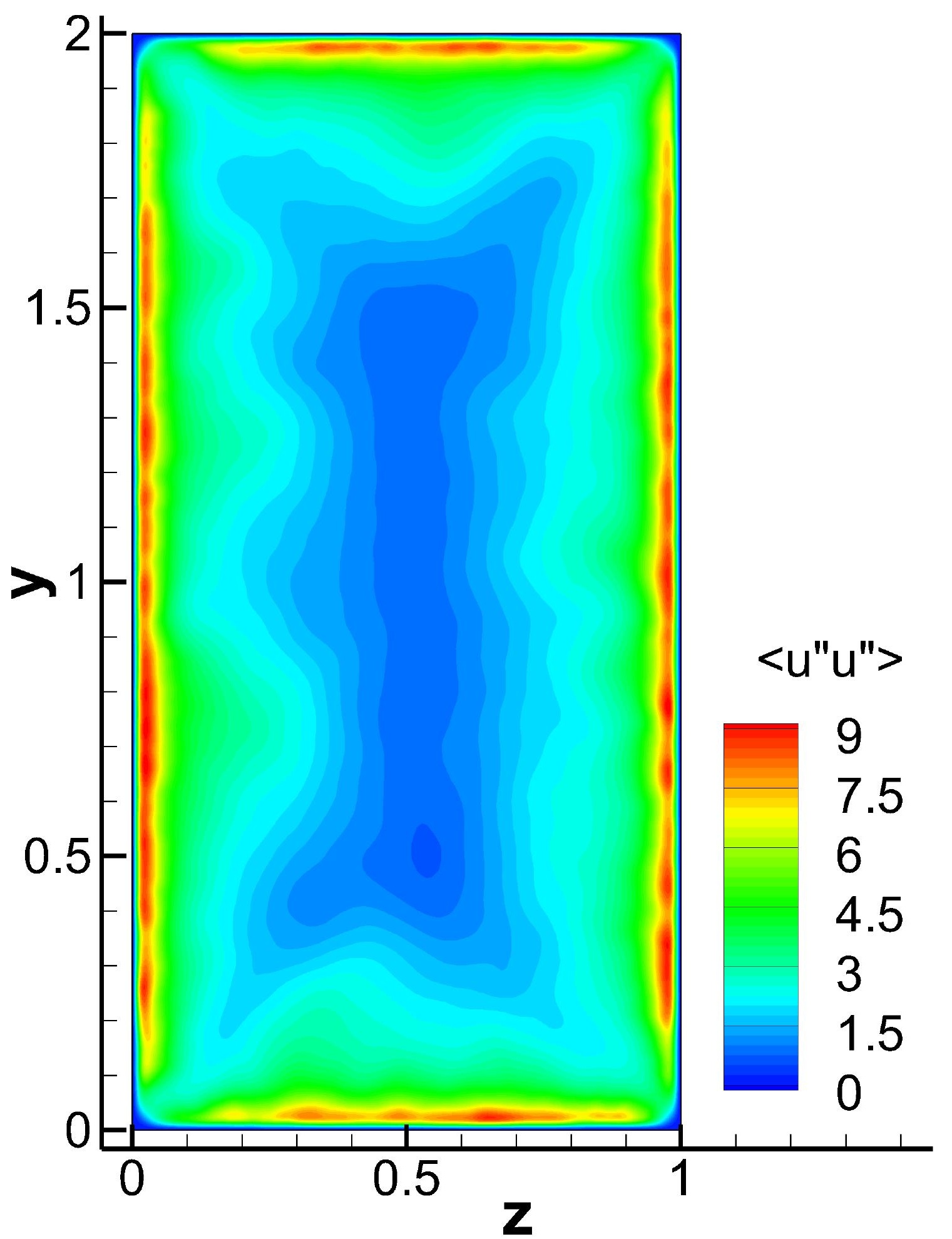




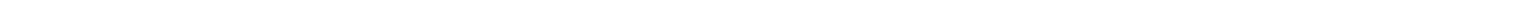




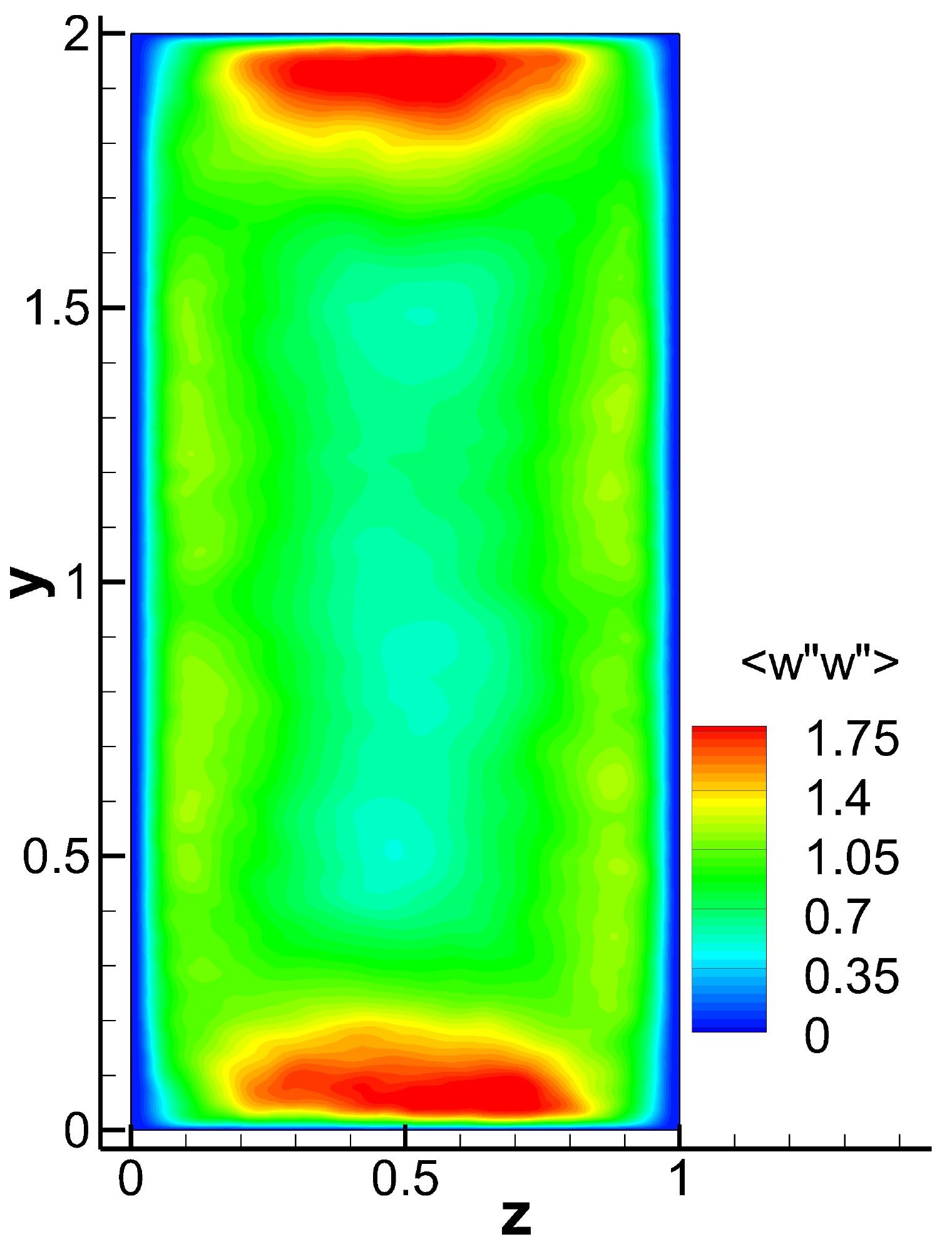




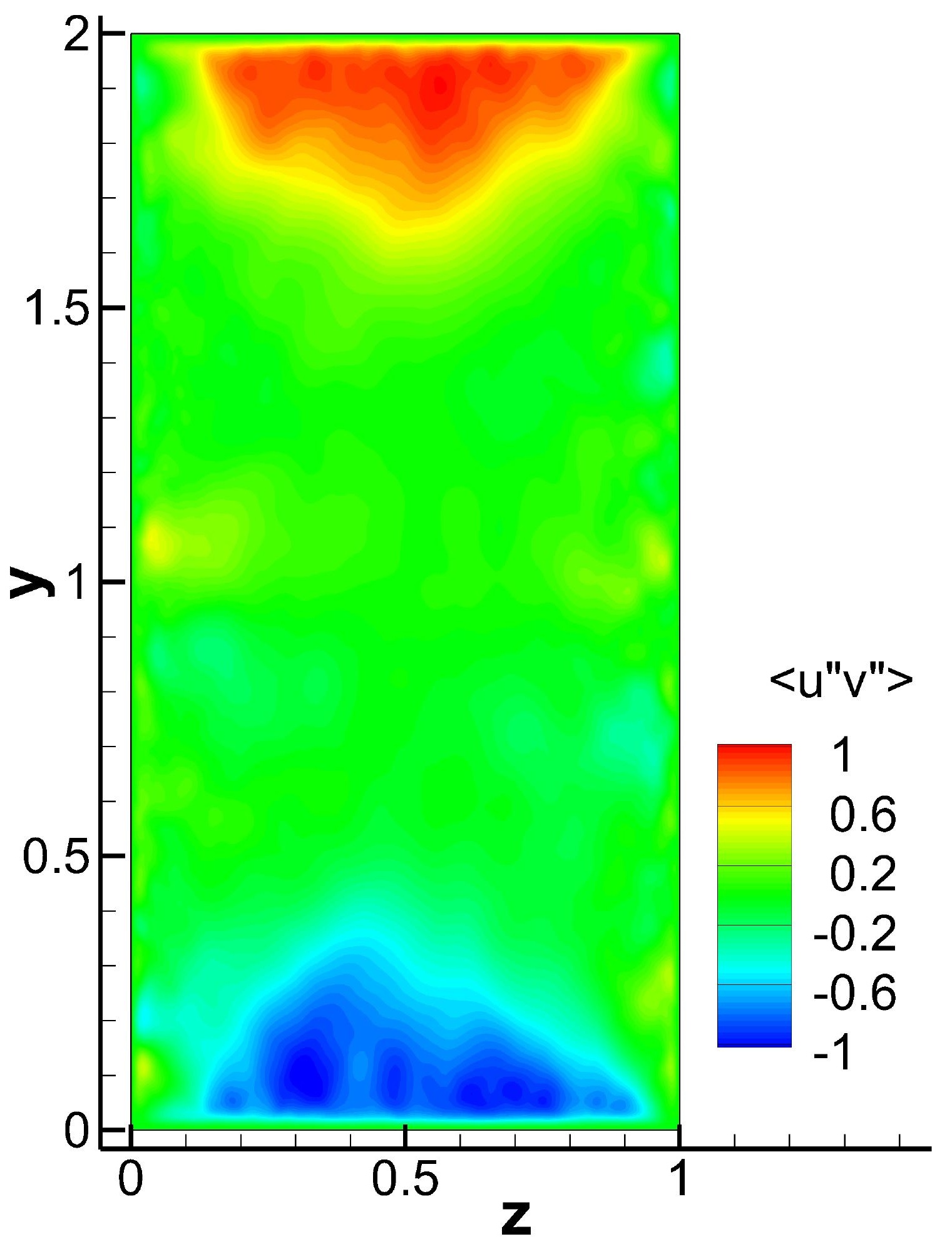




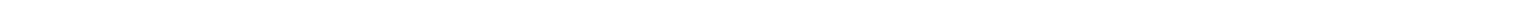




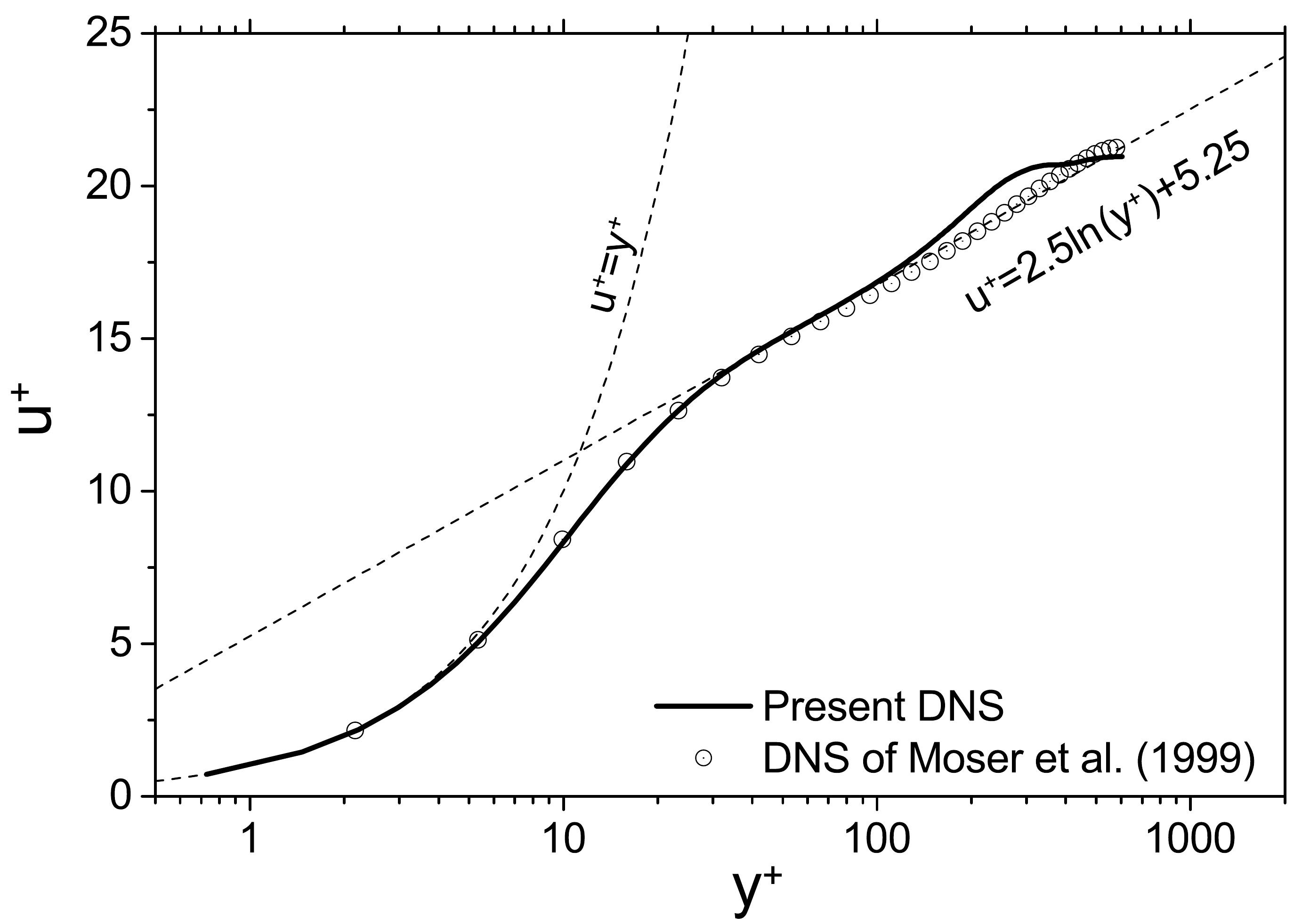




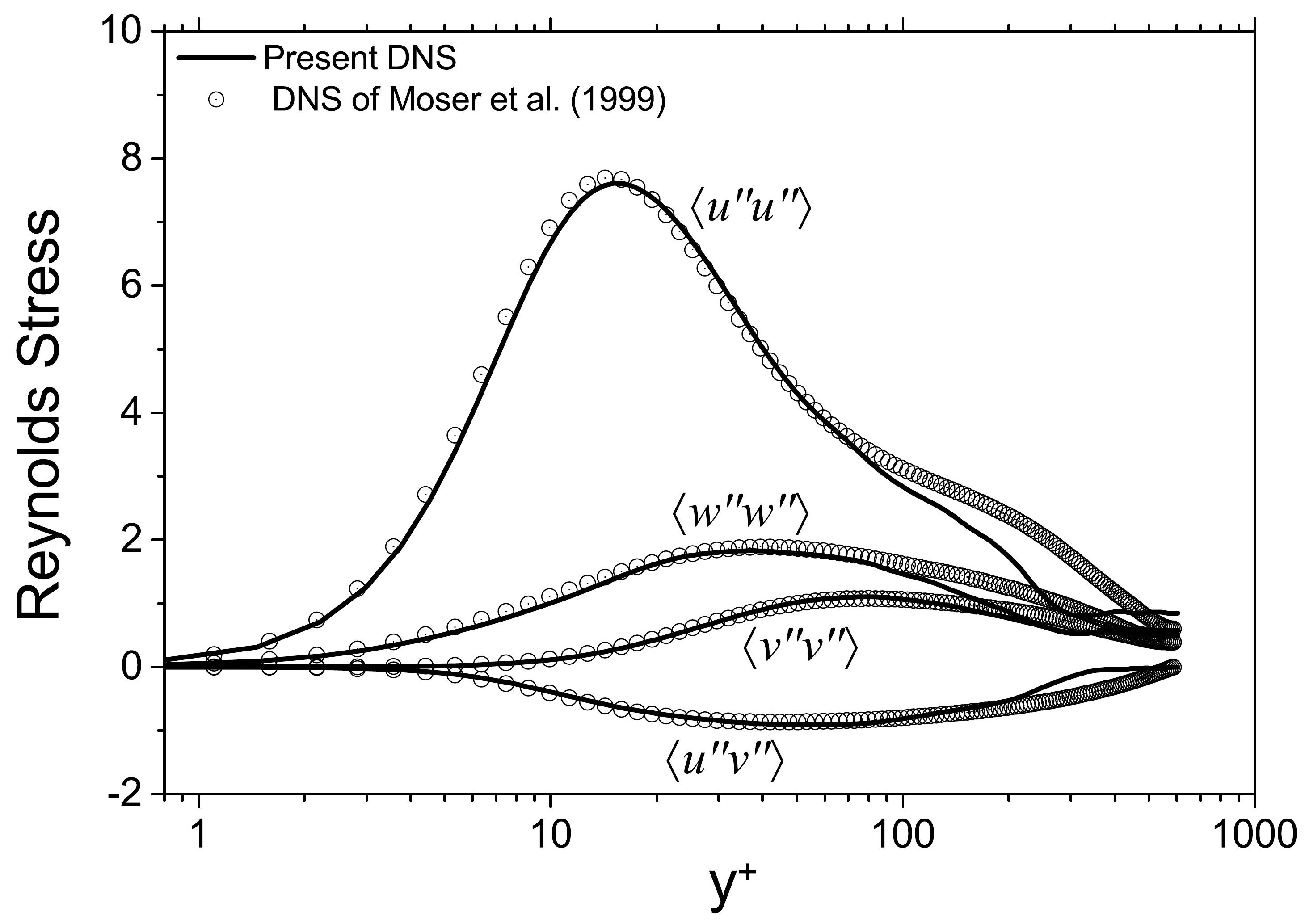




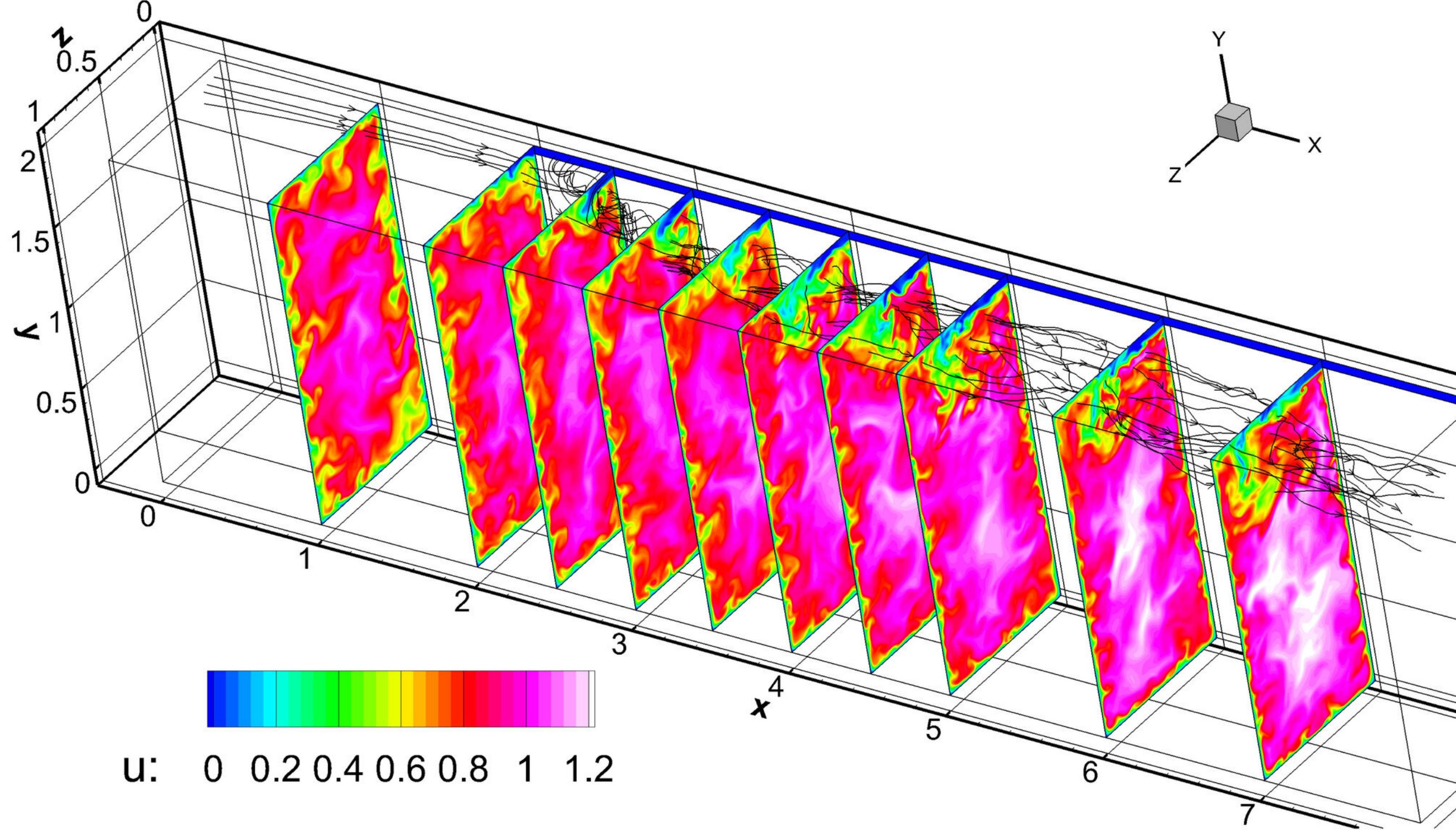




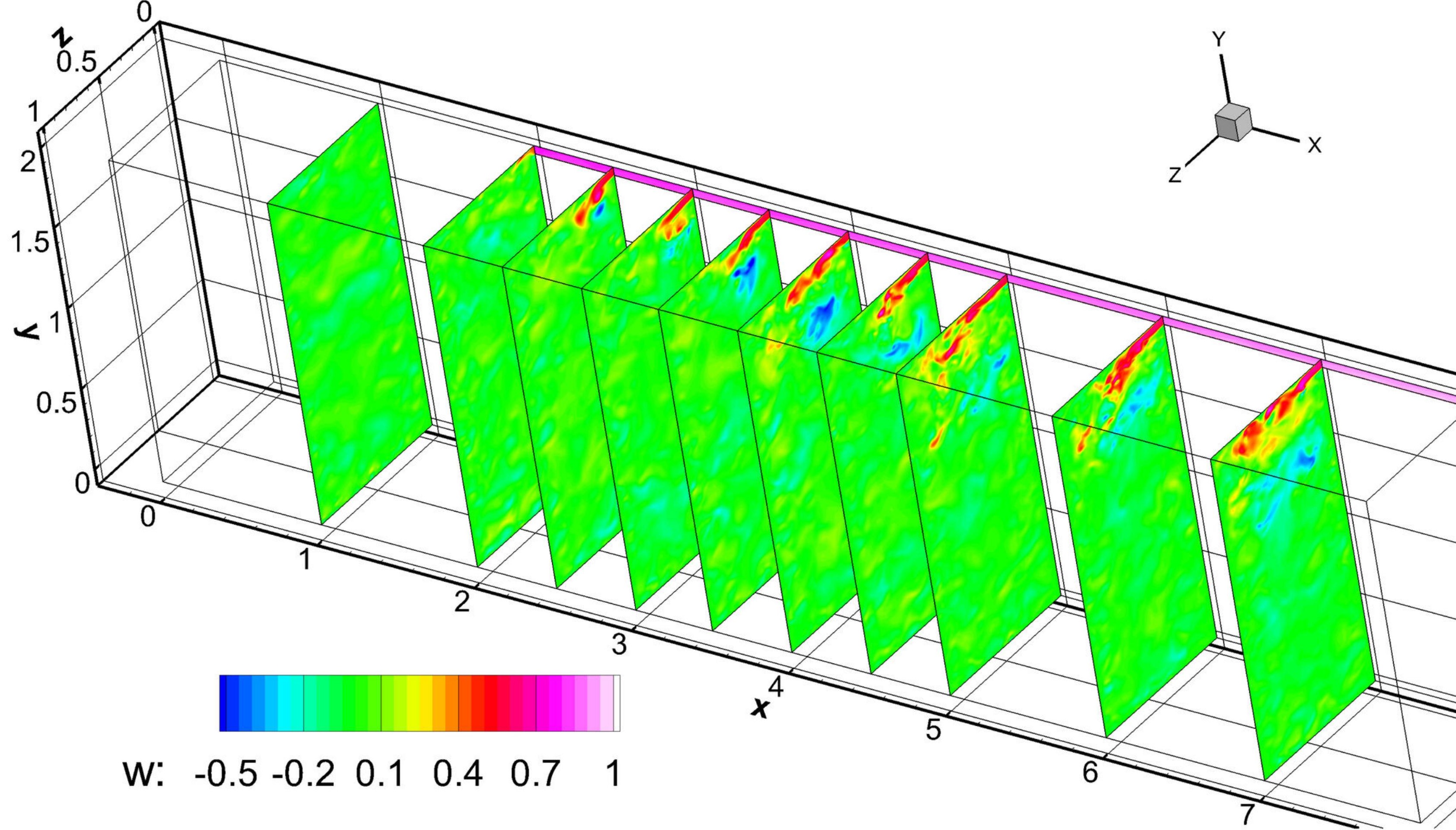




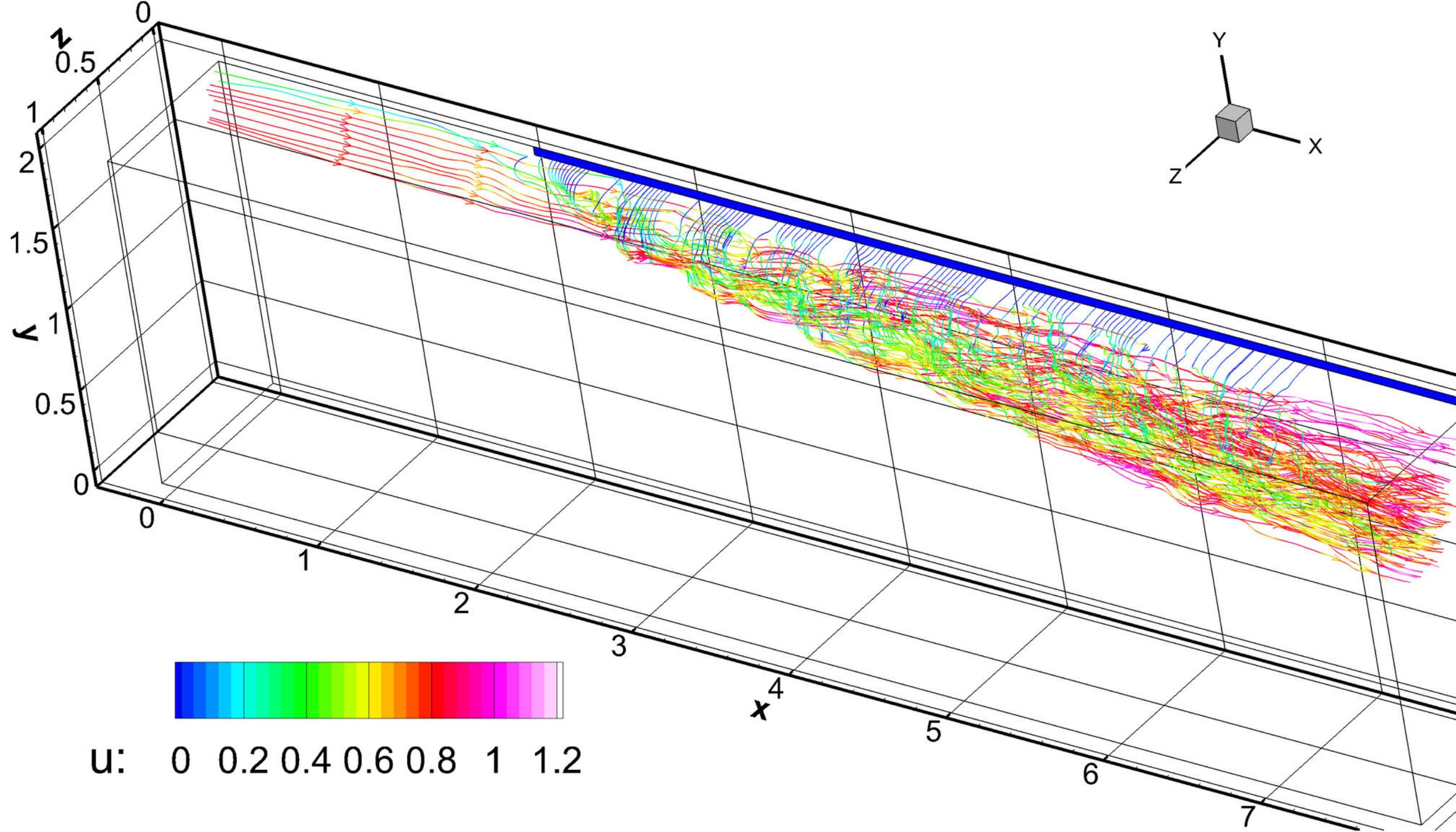




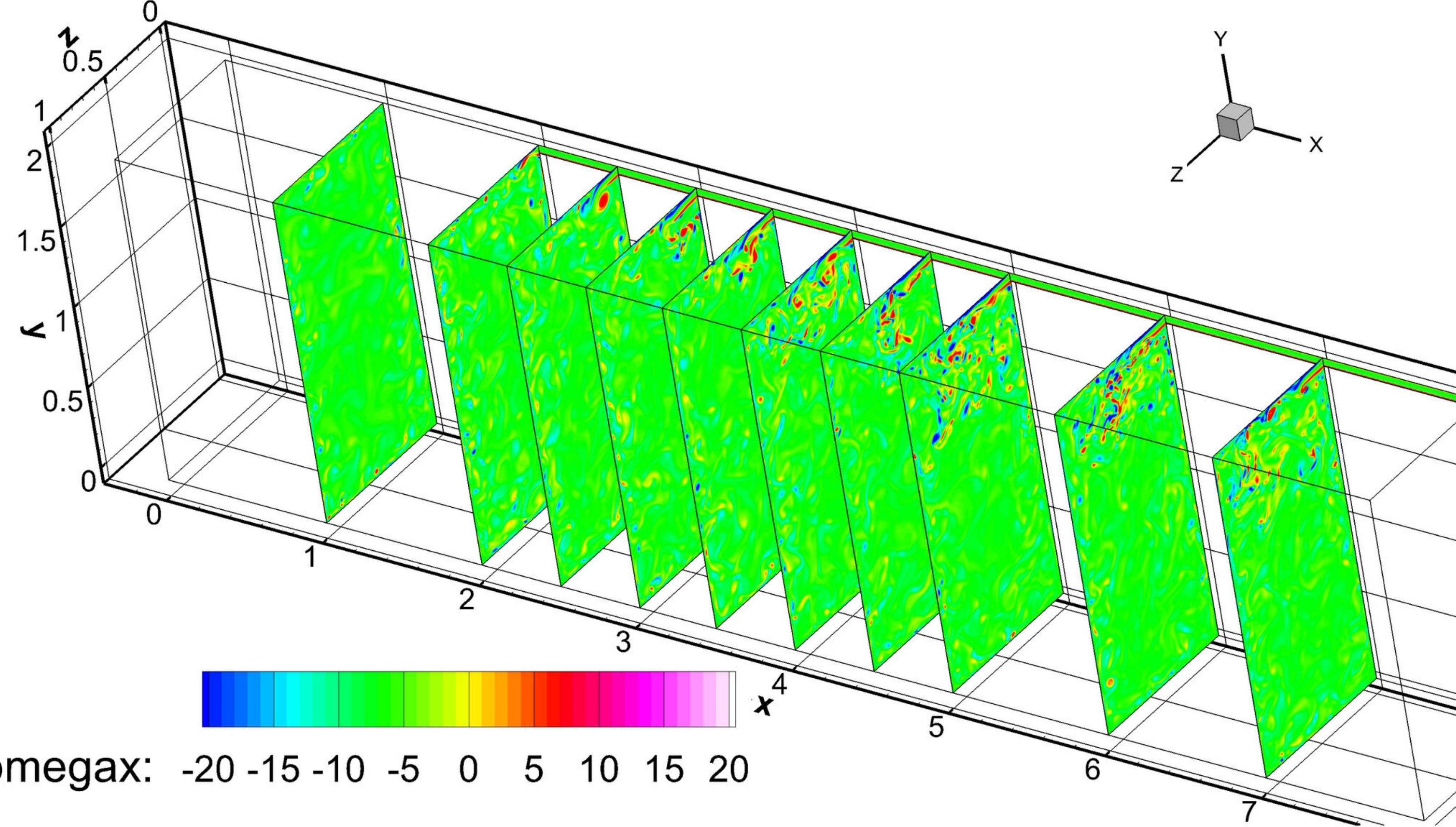




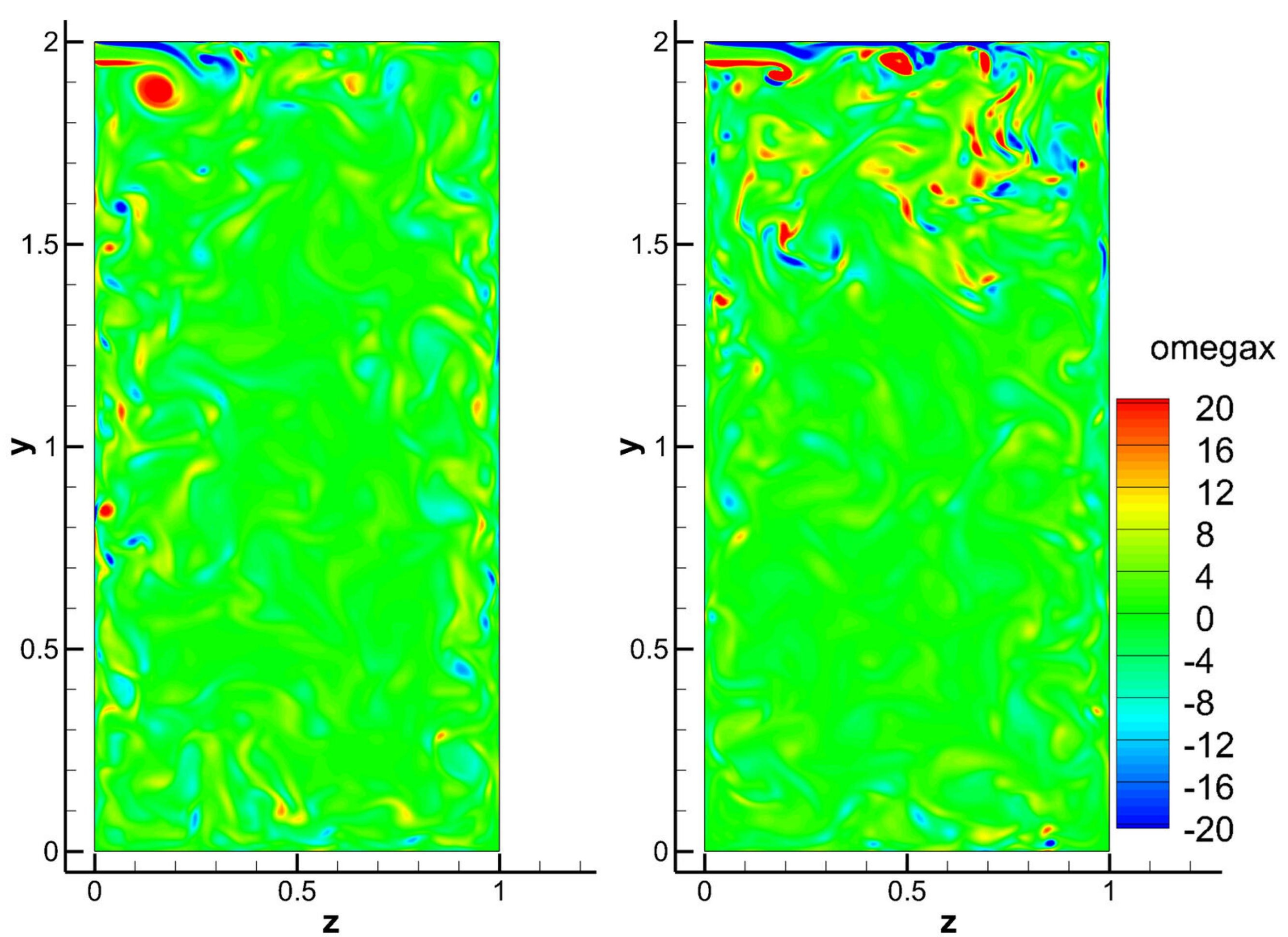




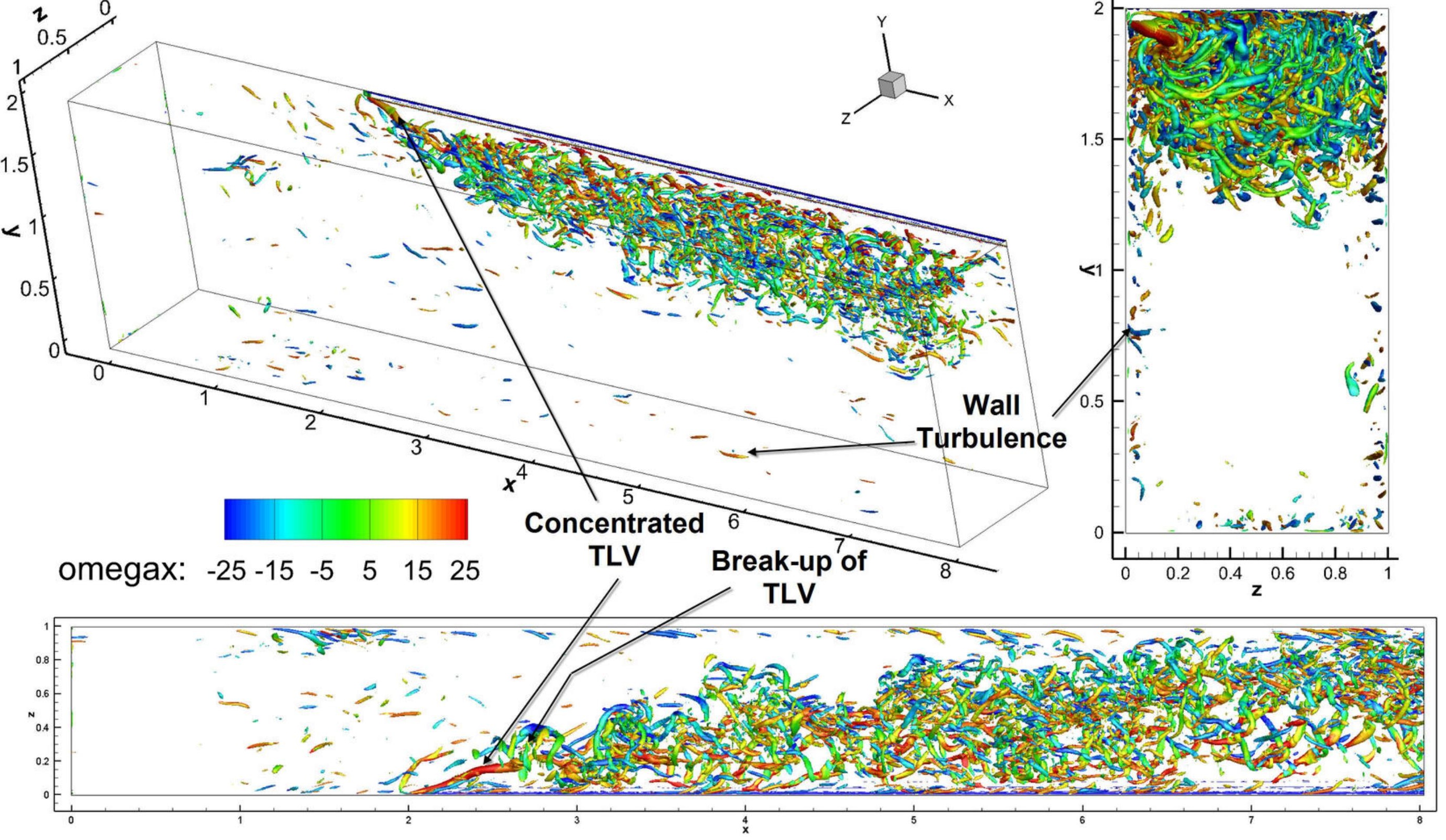




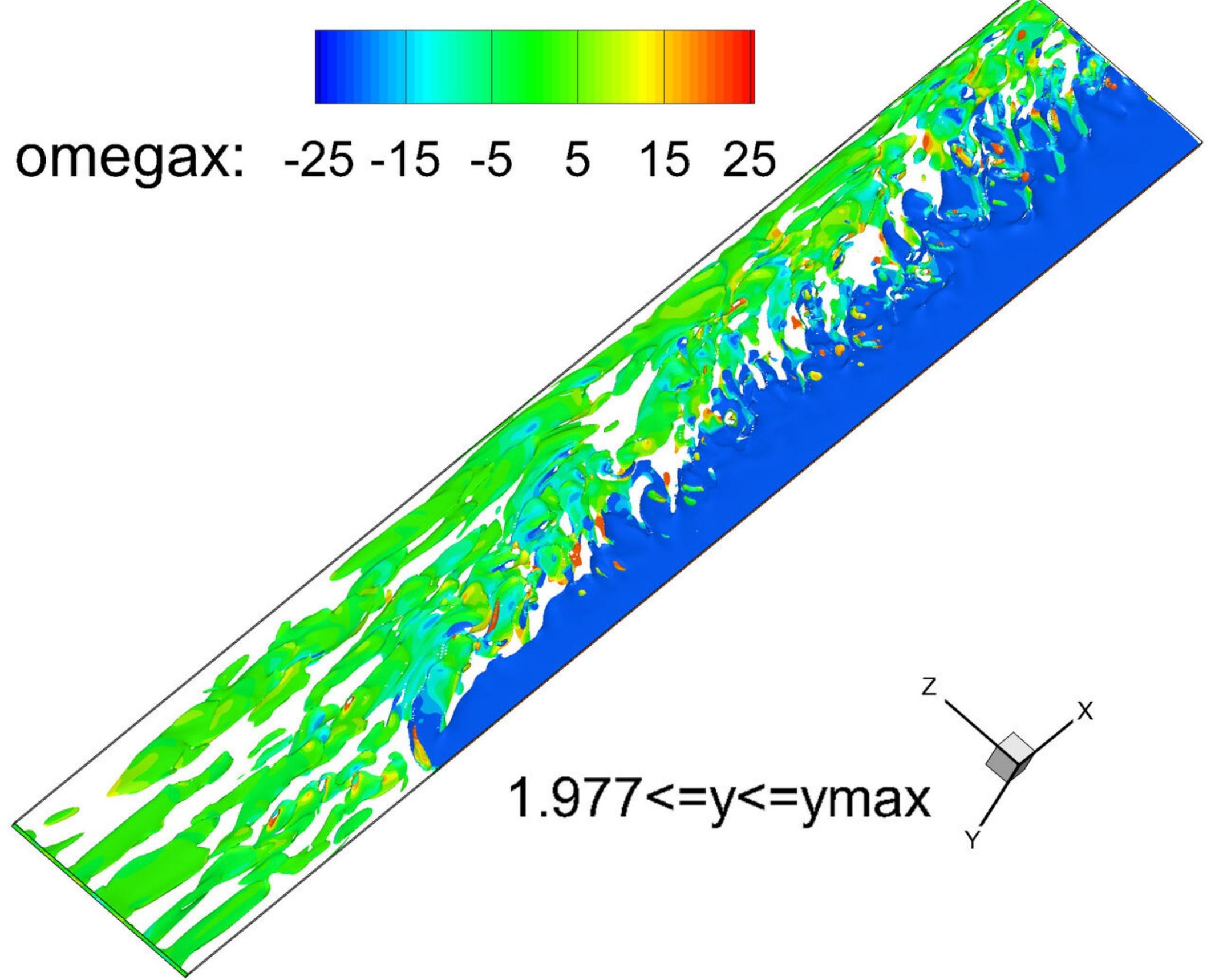




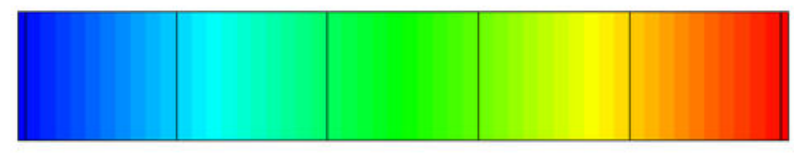 \\ omegax: $-25-15 \quad-5 \quad 5 \quad 15 \quad 25$}

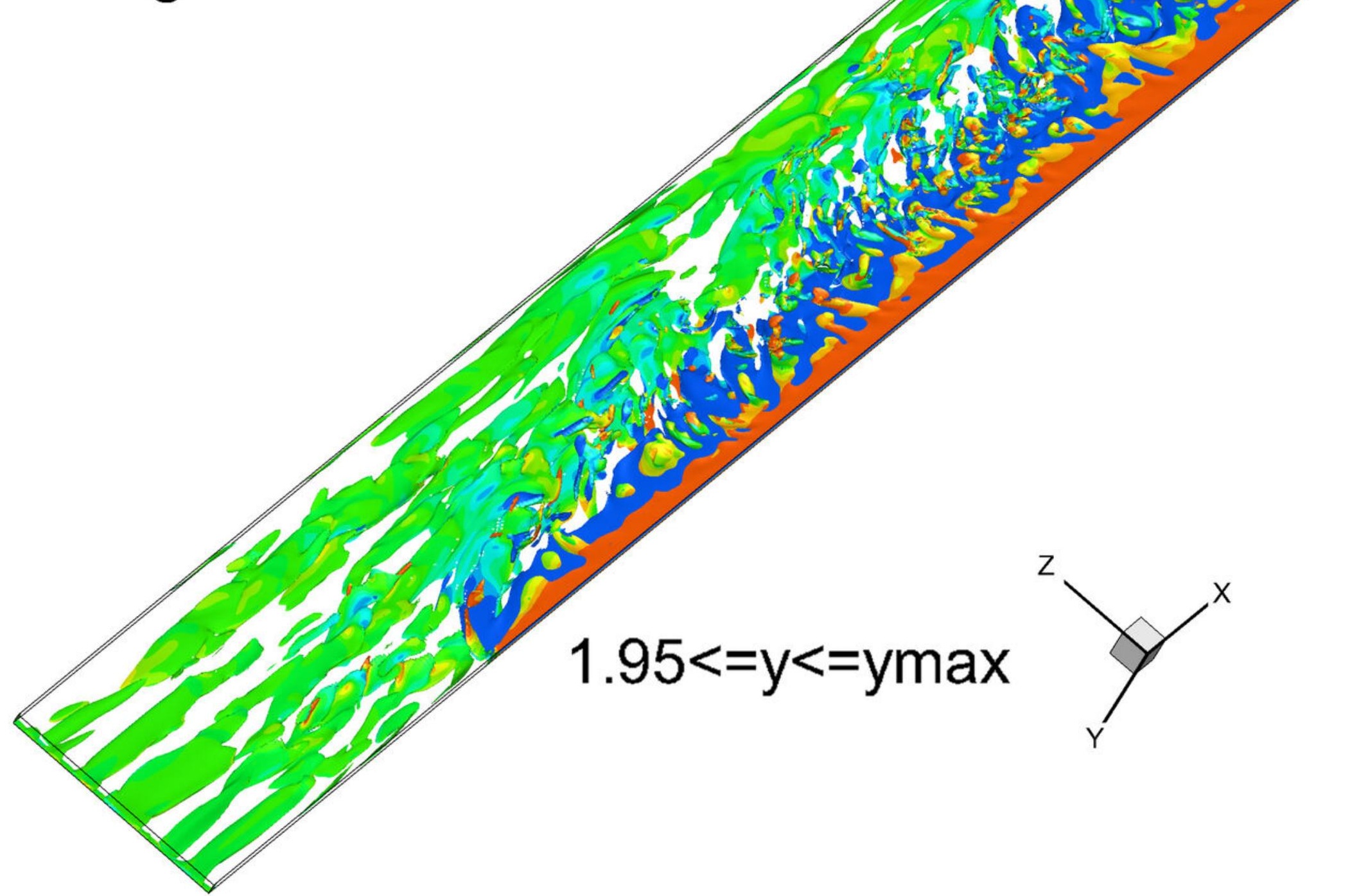




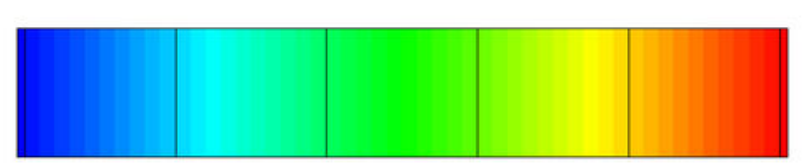

omegax: $-25-15 \quad-5 \quad 5 \quad 15 \quad 25$

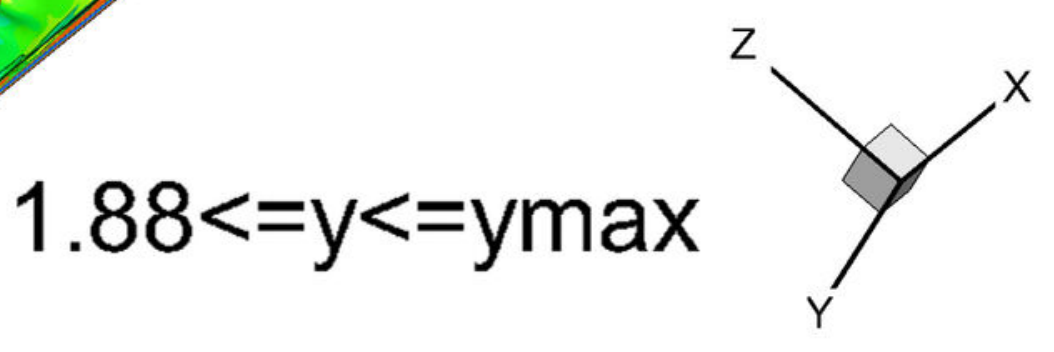




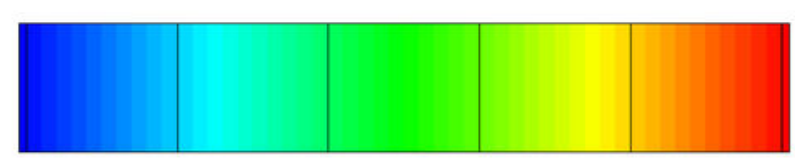

omegax: $-25-15-5 \quad 5 \quad 1525$ 


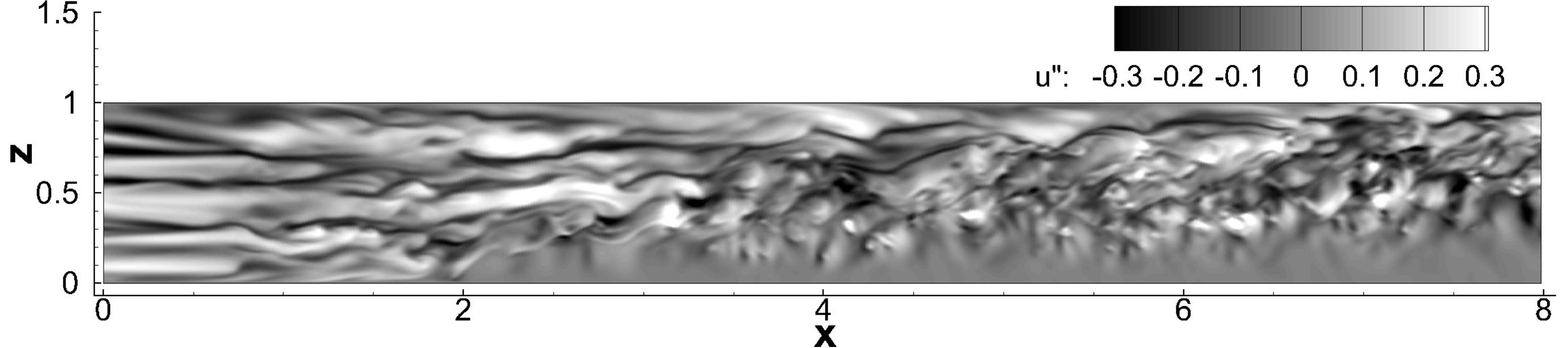




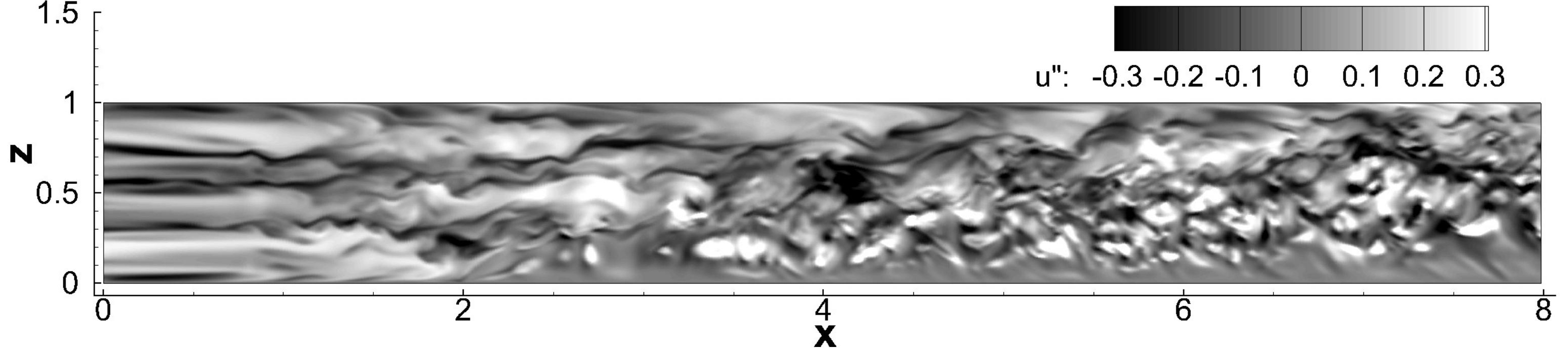




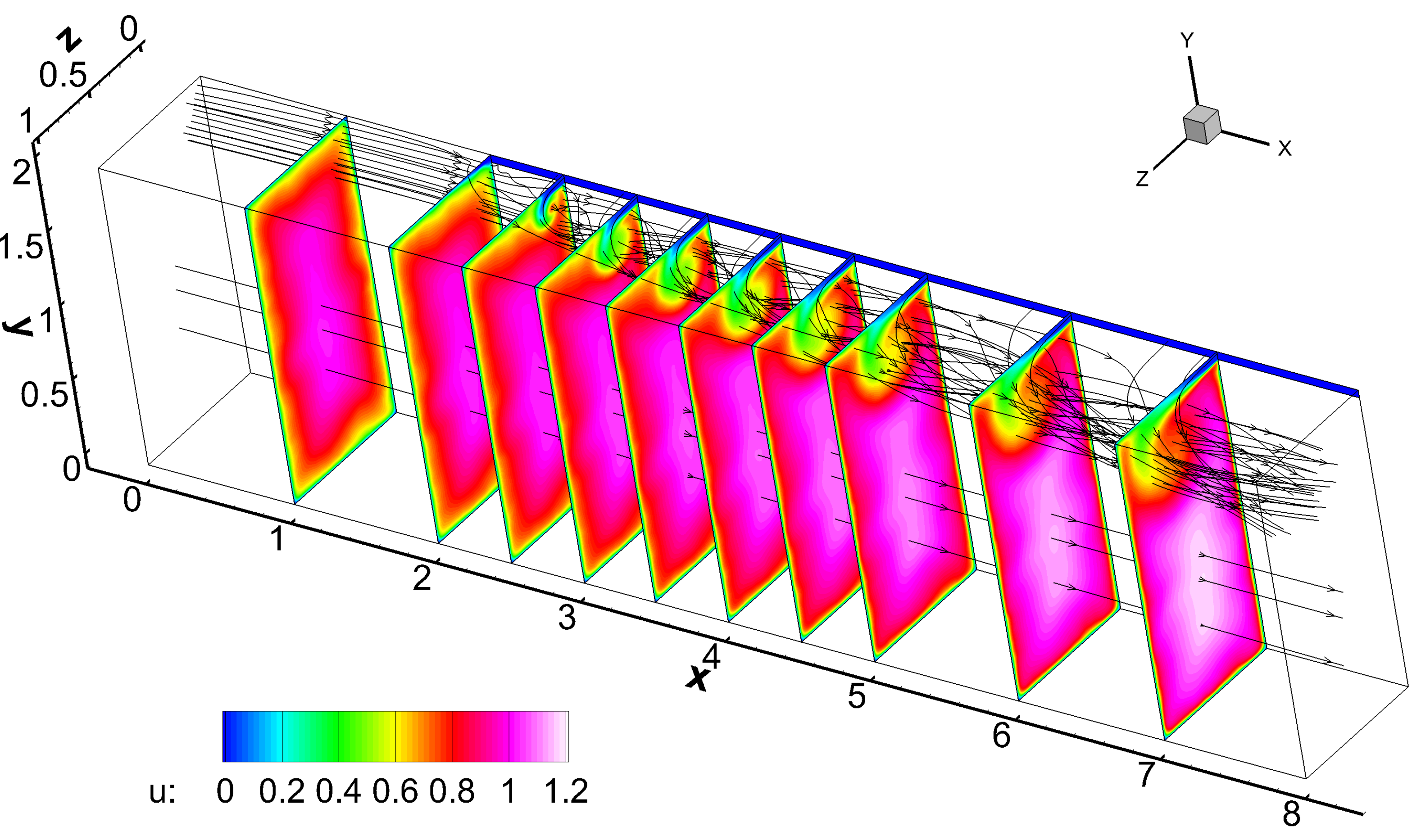




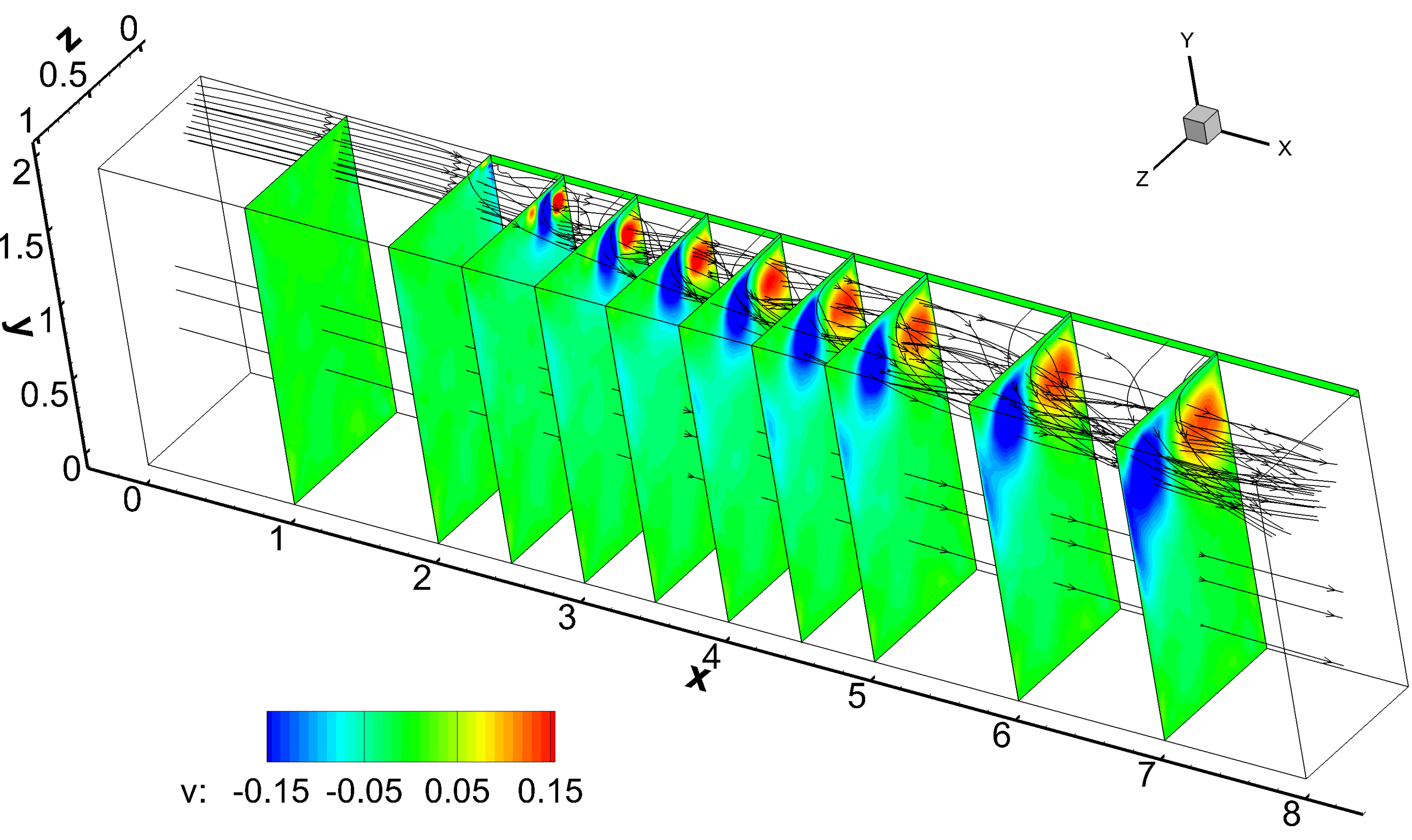




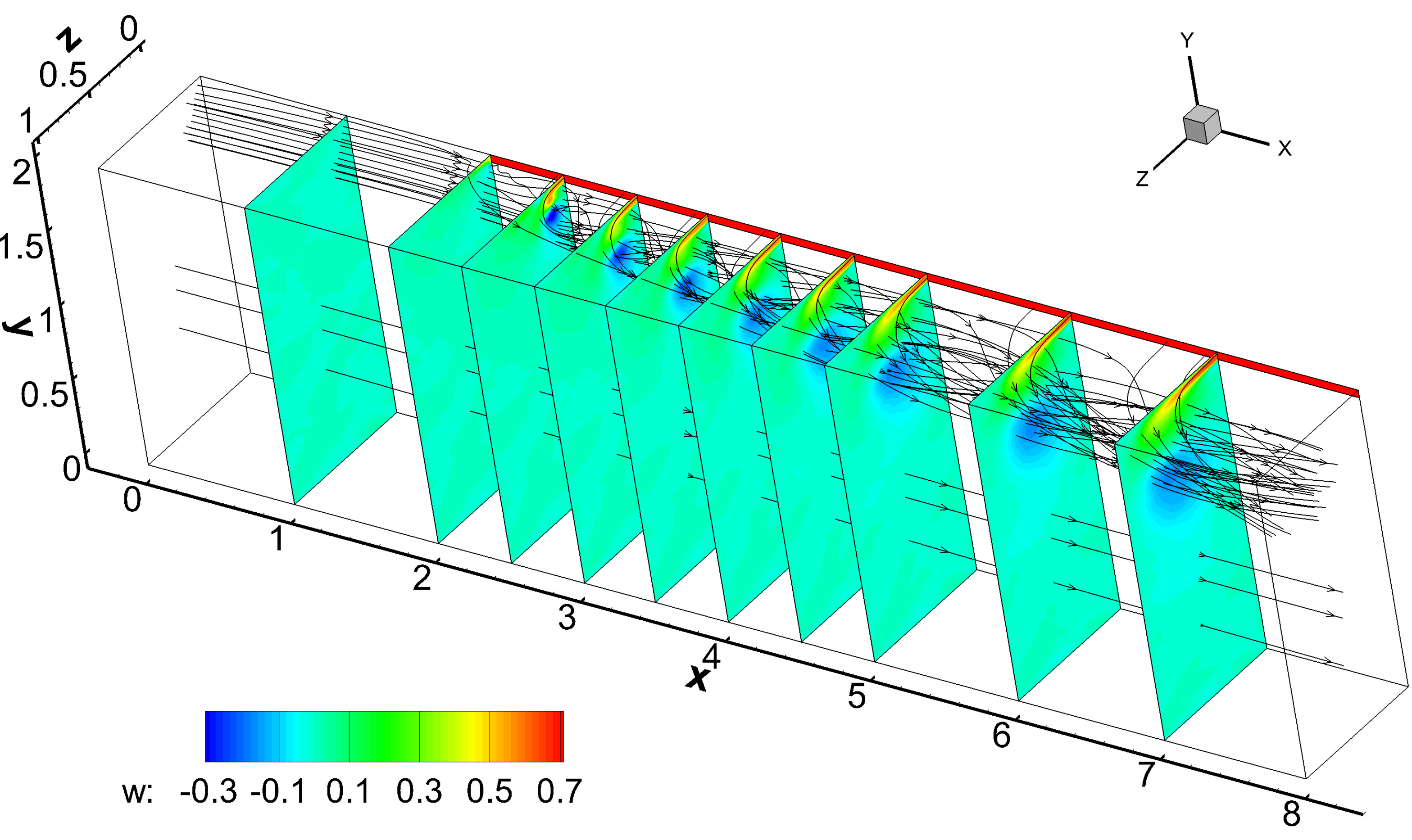




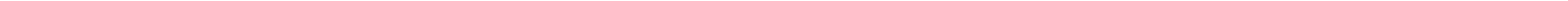




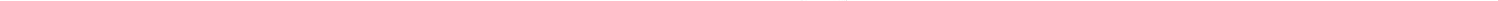




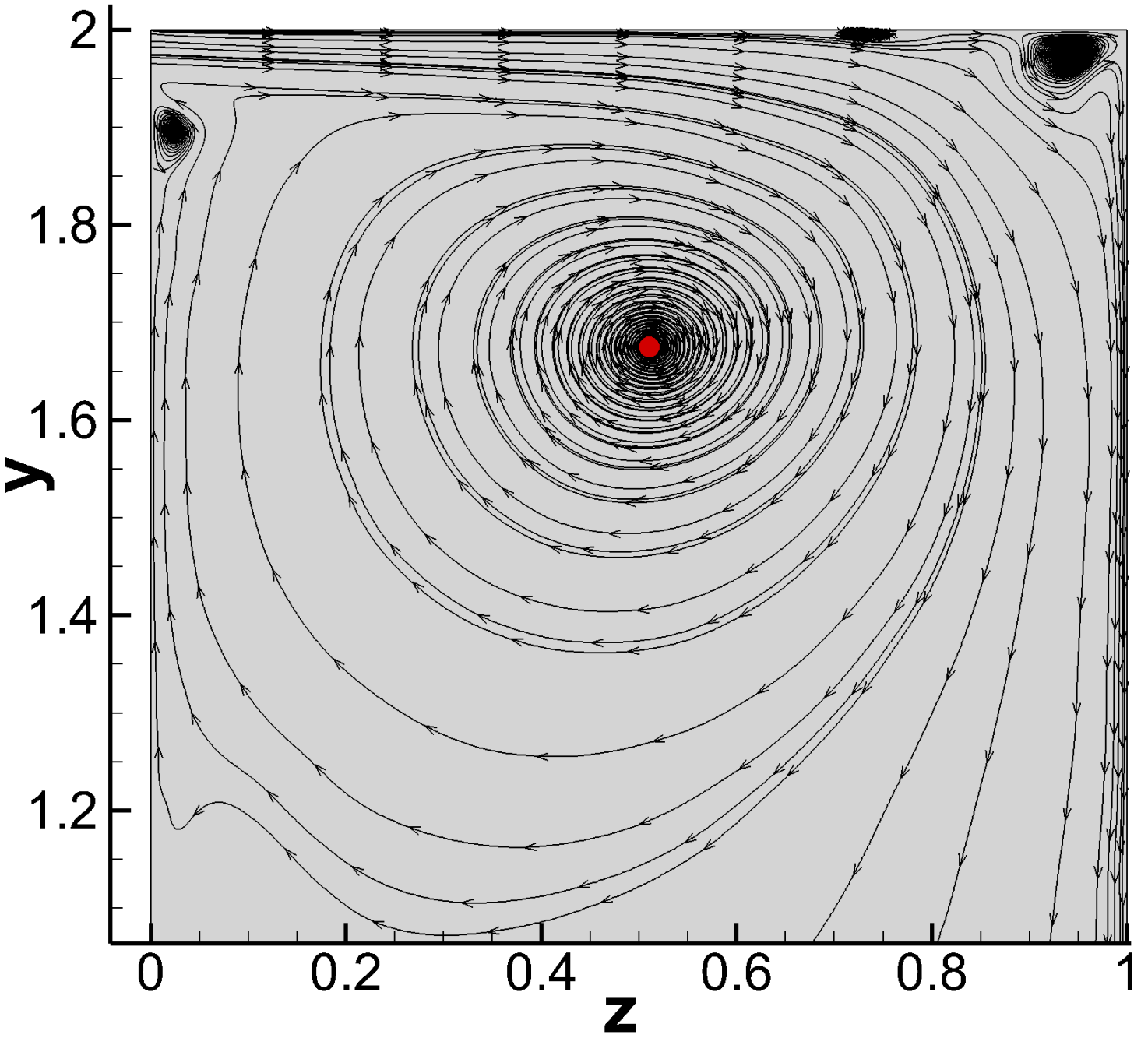




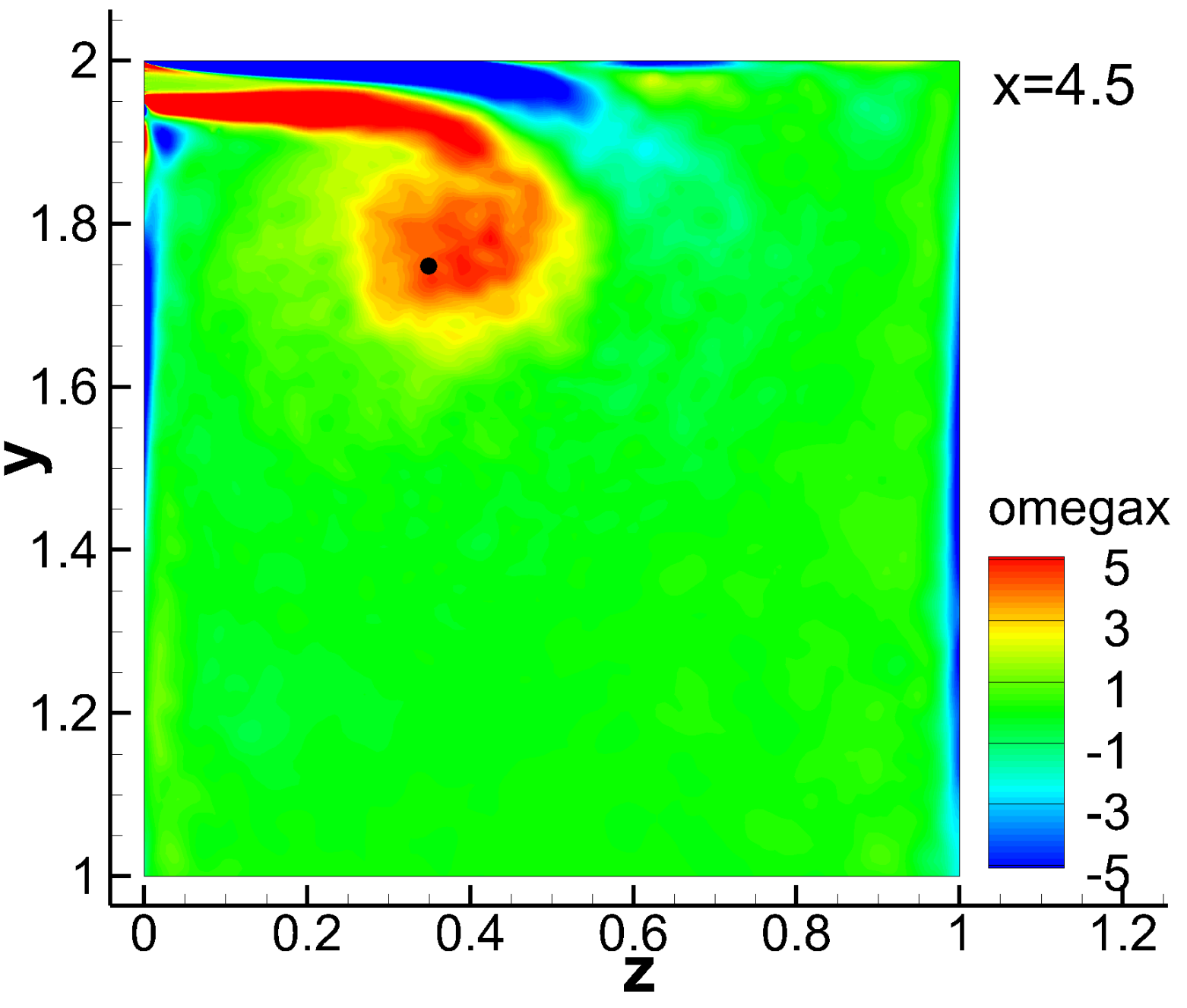




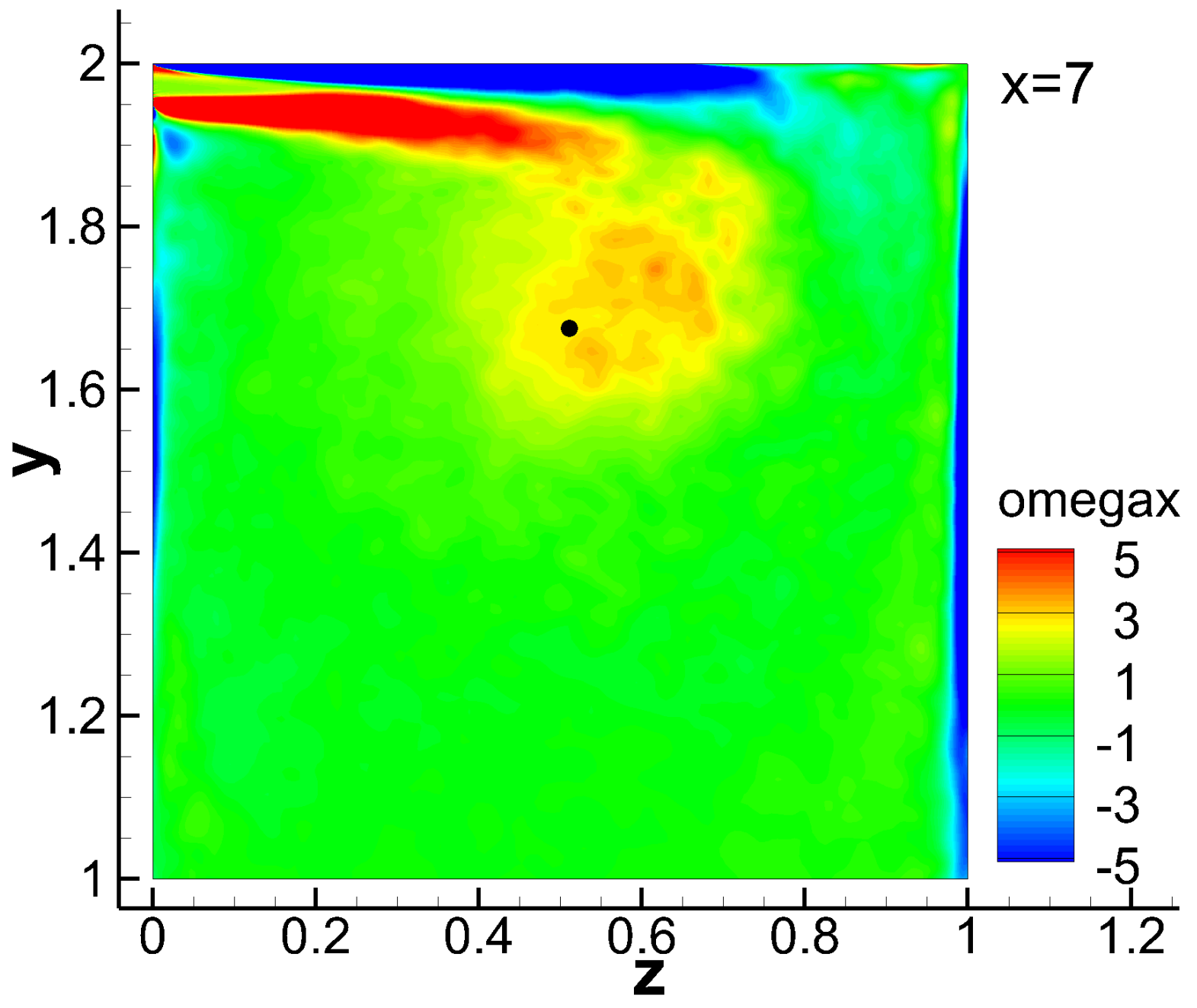




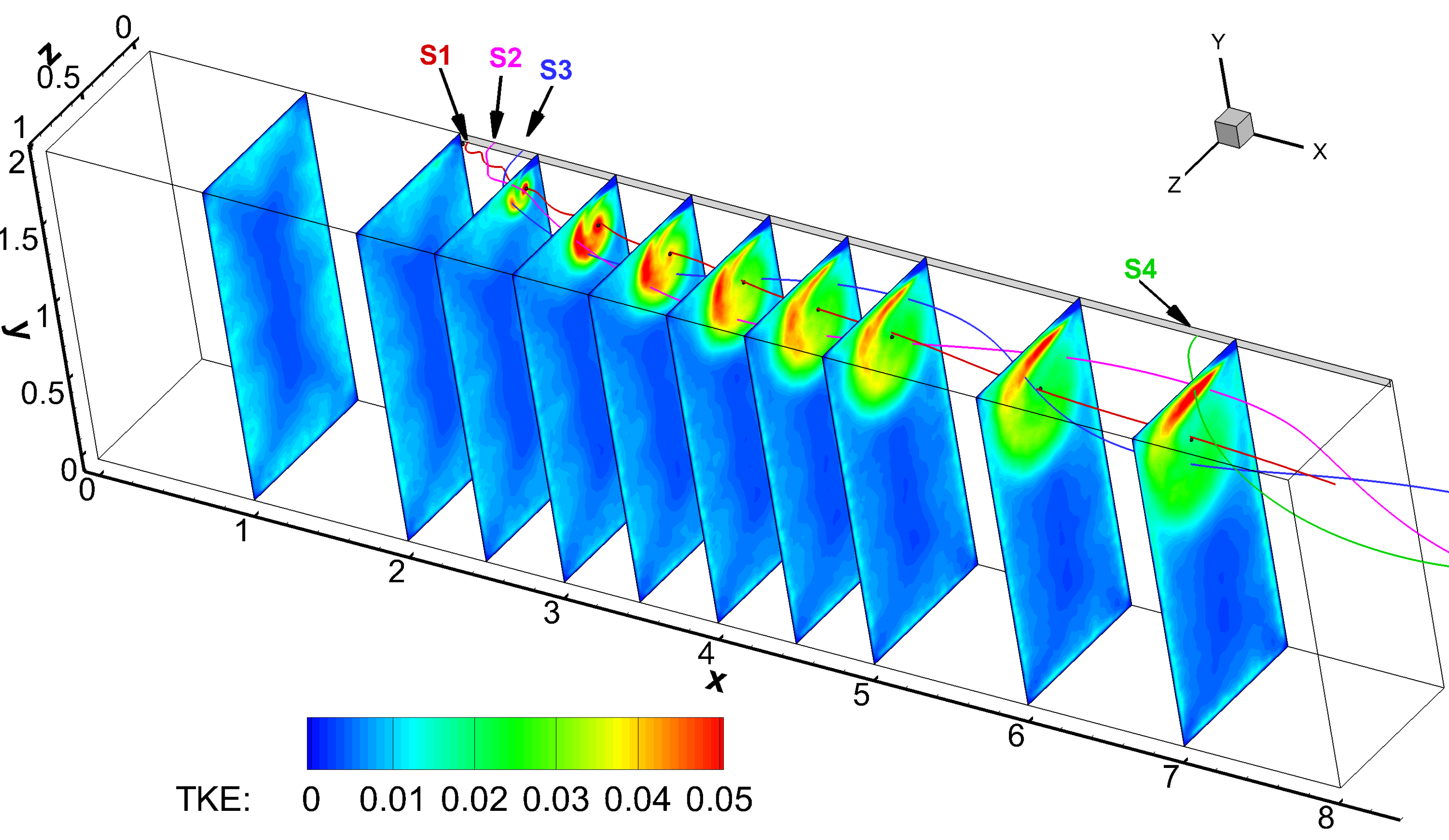




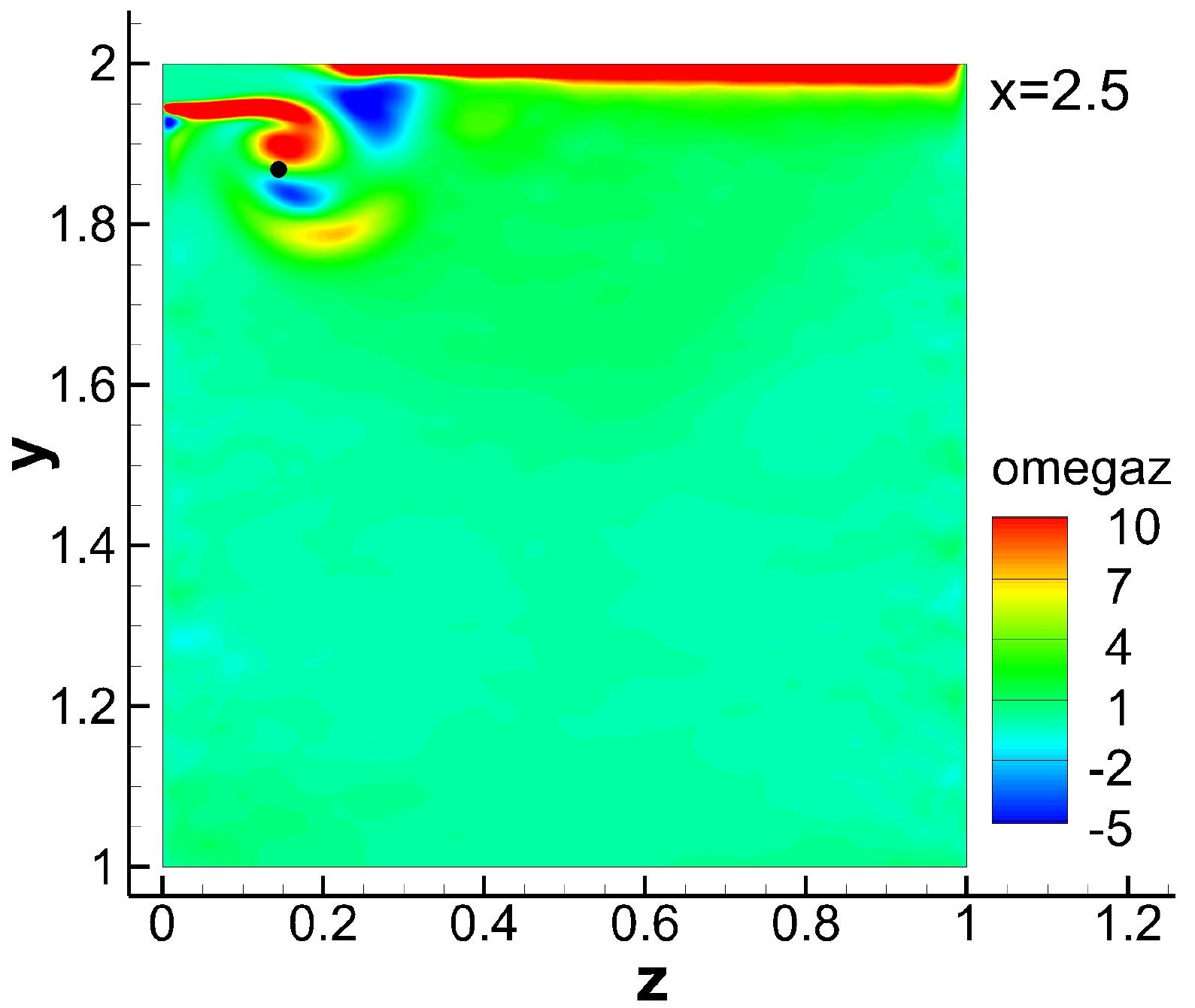




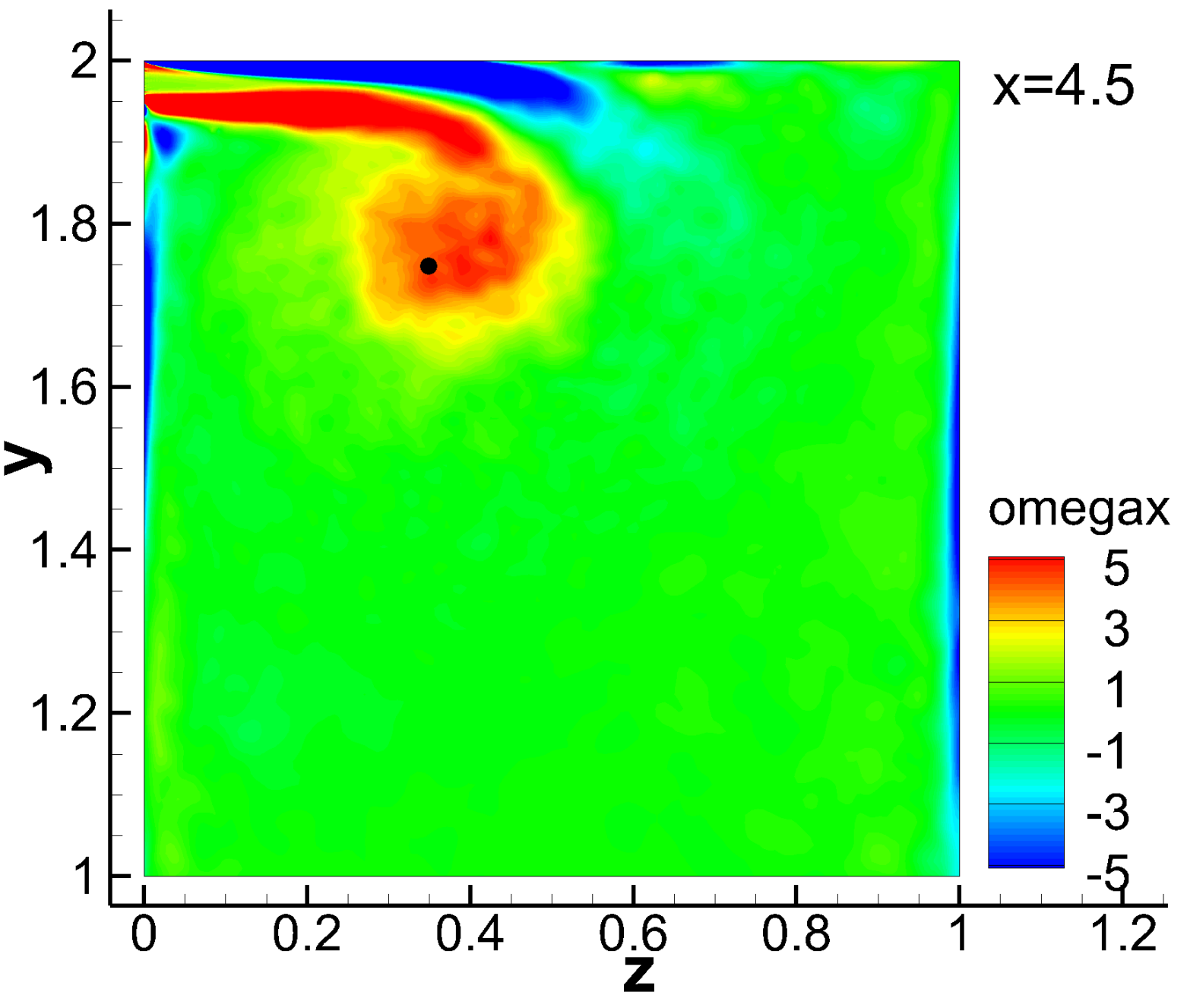




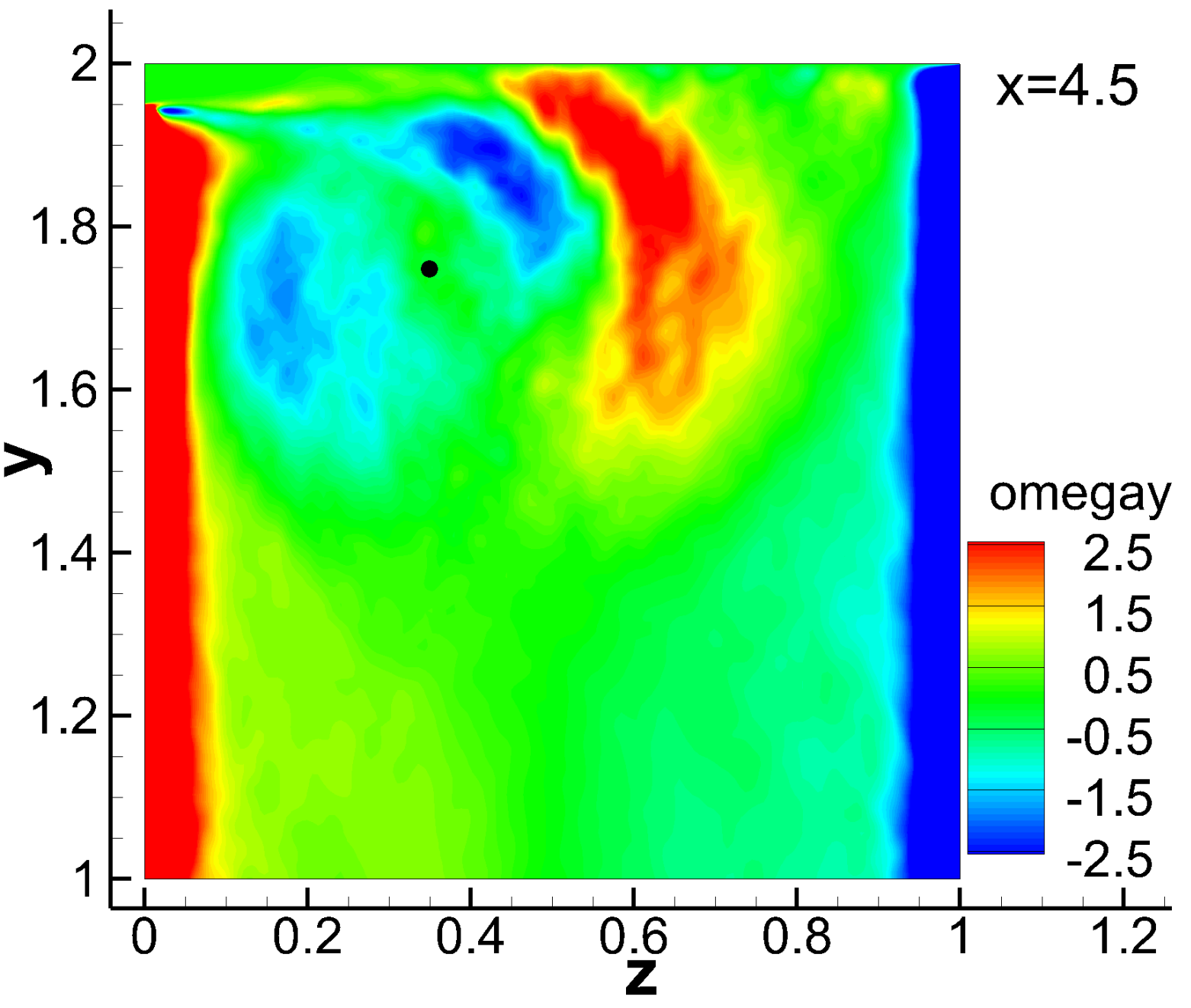




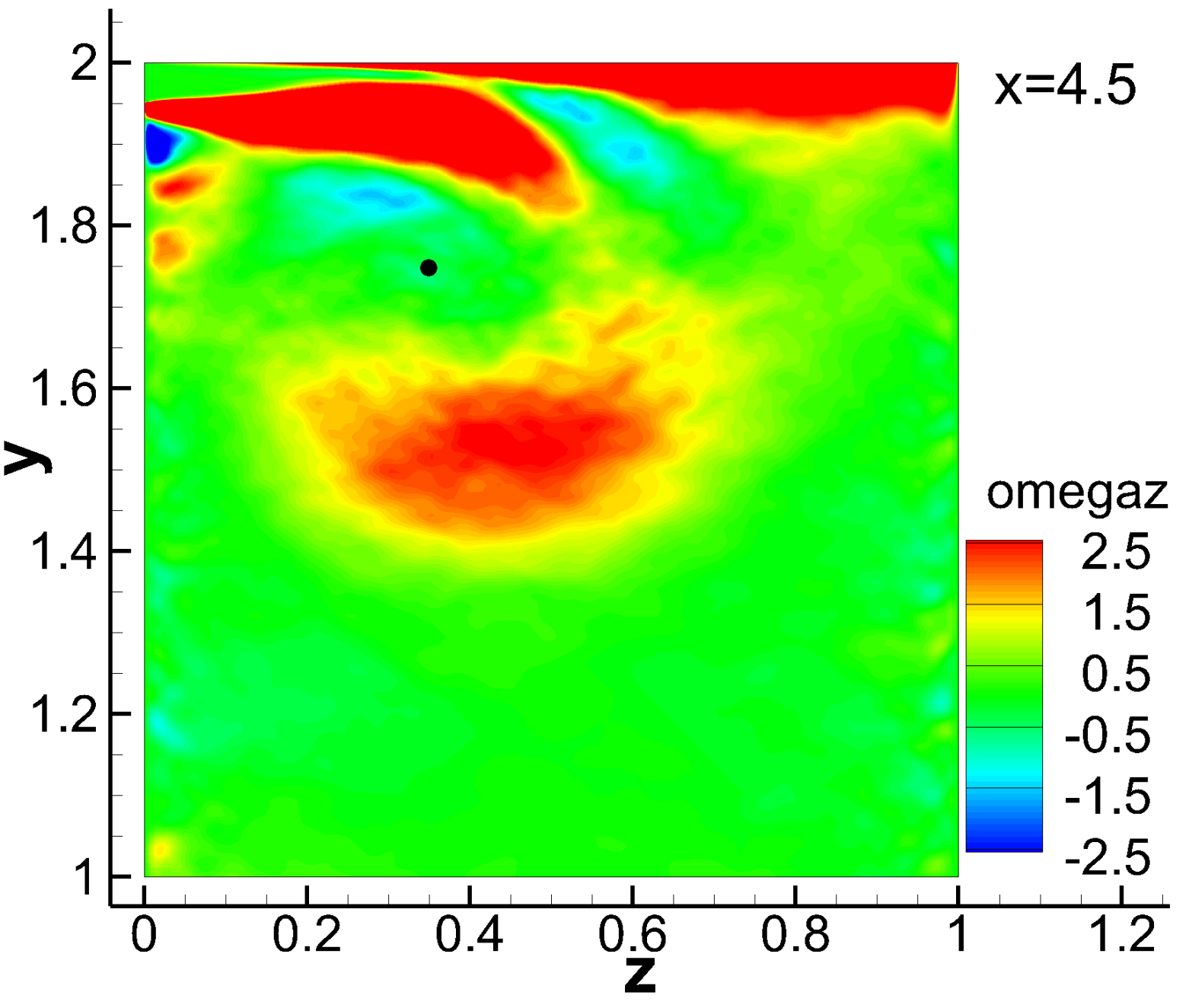




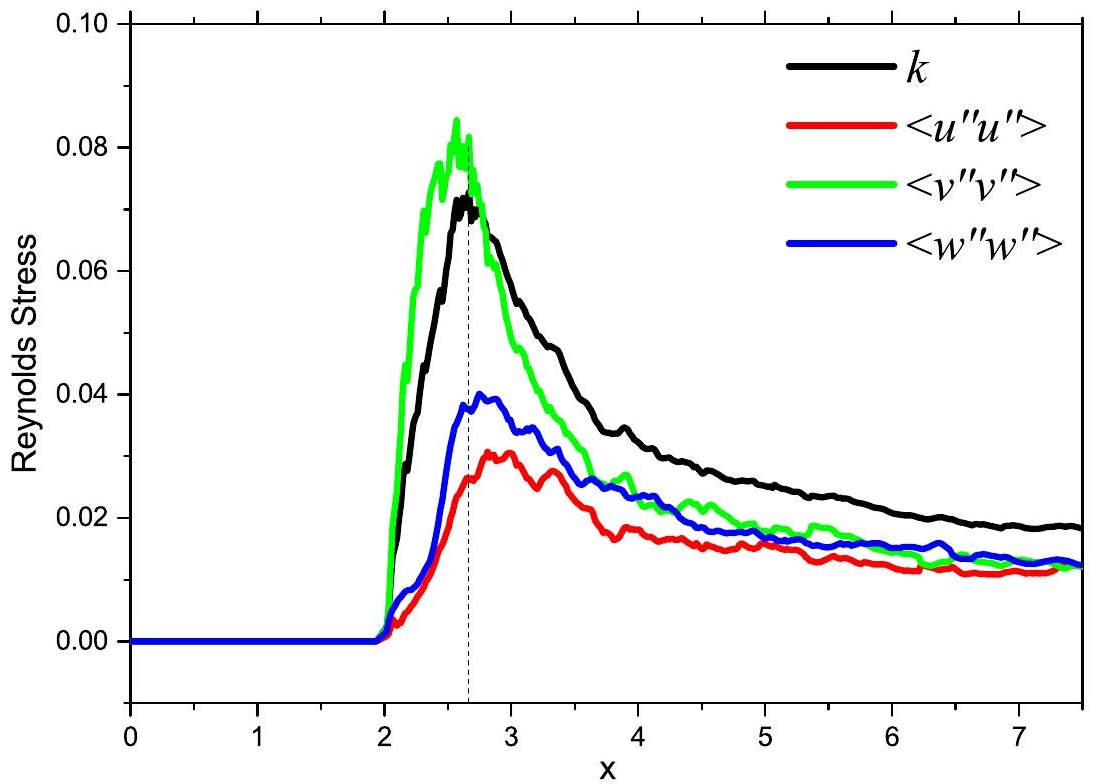




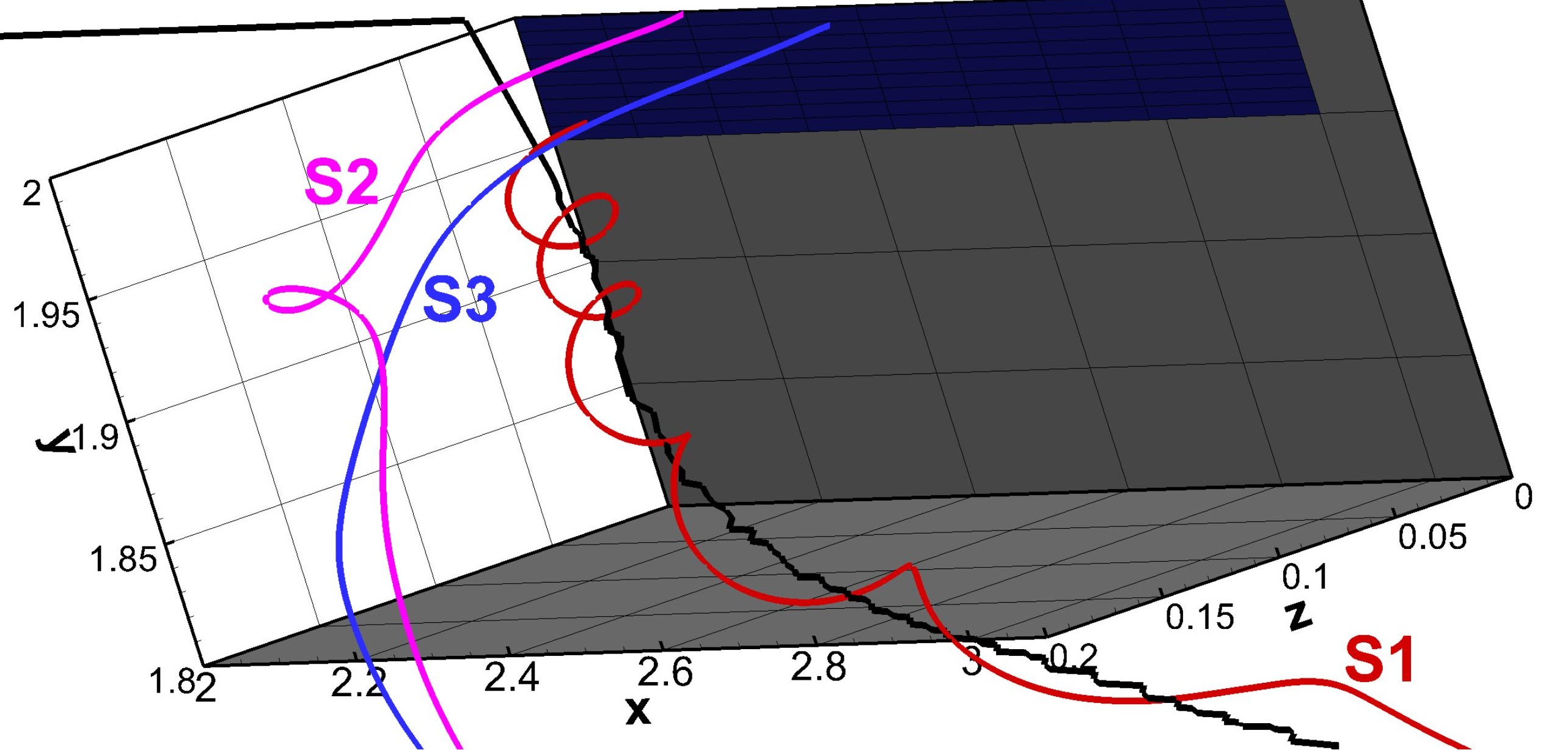




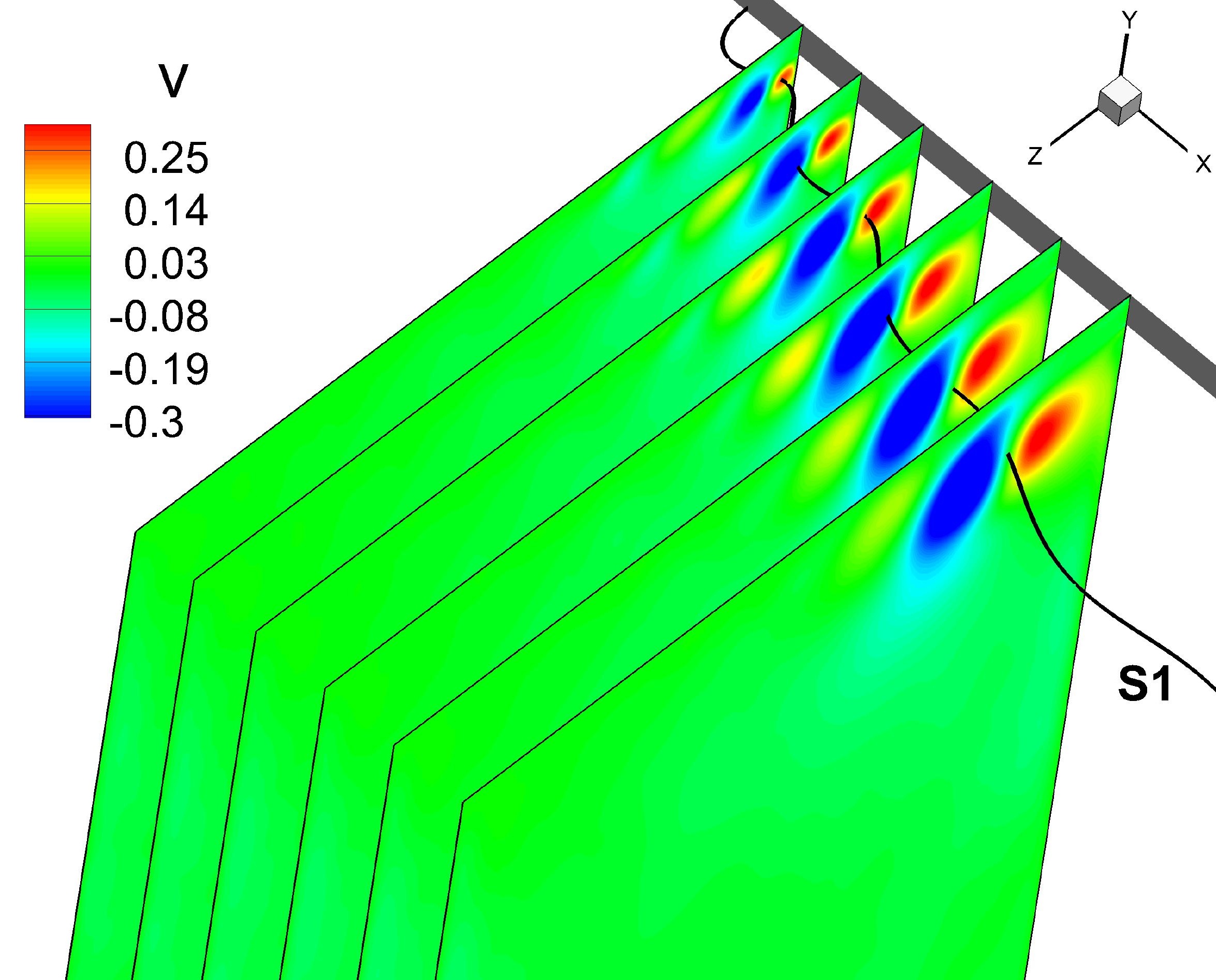




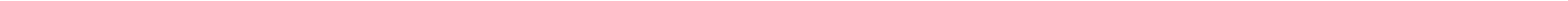




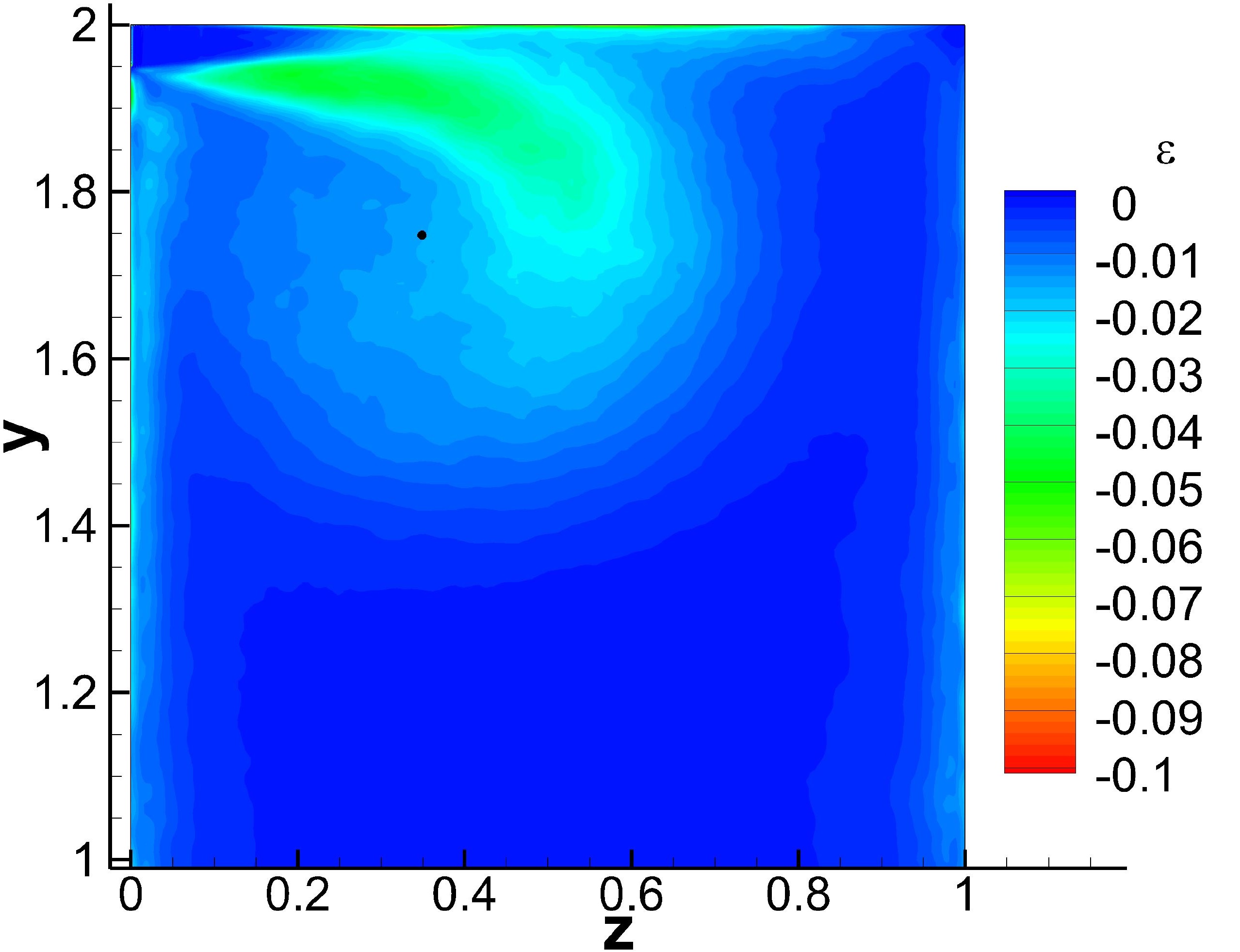




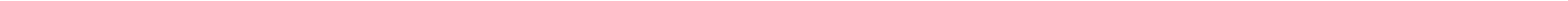




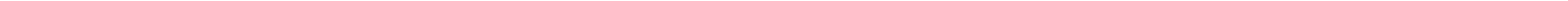




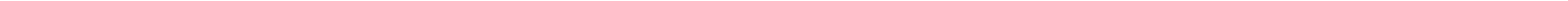

